Probabilistic Mapping of Spatial Motion Patterns for Mobile Robots 

Örebro Studies in Technology 80

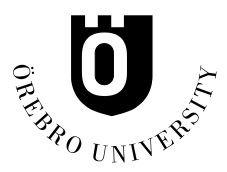

TOMASZ PIOTR KUCNER

\section{Probabilistic Mapping of Spatial Motion Patterns for Mobile Robots}


(C) Tomasz Piotr Kucner, 2018

Title: Probabilistic Mapping of Spatial Motion Patterns for Mobile Robots

Publisher: Örebro University 2018 www.oru.se/publikationer-avhandlingar

Print: Örebro University, Repro 08/2018

ISSN $1650-8580$

ISBN 978-91-7529-255-7 


\section{Abstract}

Tomasz Piotr Kucner (2018): Probabilistic Mapping of Spatial Motion Patterns for Mobile Robots. Örebro Studies in Technology 80.

To bring robots closer to real-world autonomy, it is necessary to equip them with tools allowing them to perceive, model and behave adequately to dynamic changes in the environment. The idea of incorporating information about dynamics not only in the robots reactive behaviours but also in global planning process stems from the fact that dynamic changes are typically not completely random and follow spatiotemporal patterns. The overarching idea behind the work presented in this thesis is to investigate methods allowing to represent the variety of the real-world spatial motion patterns in a compact, yet expressive way. The primary focus of the presented work is on building maps capturing the motion patterns of dynamic objects and/or the flow of continuous media.

The contribution of this thesis is twofold. First, I introduce Conditional-Transition Map: a representation for modelling motion patterns of dynamic objects as a multimodal flow of occupancy over a grid map. Furthermore, in this thesis I also propose an extension (Temporal Conditional-Transition Map), which models the speed of said flow. The proposed representations connect the changes of occupancy among adjacent cells. Namely, they build conditional models of the direction to where occupancy is heading given the direction from which the occupancy arrived. Previously, all of the representations modelling dynamics in grid maps assumed cell independence. The representations assuming cell independence are substantially less expressive and store only information about the observed levels of dynamics (i.e. how frequent changes are at a certain location). In contrast, the proposed representations also encode information about the direction of motion. Furthermore, the multimodal and conditional character of the representations allows to distinguish and correctly model intersecting flows. The capabilities of the introduced grid-based representations are demonstrated with experiments performed on real-world data sets.

In the second part of this thesis, I introduce Circular Linear Flow Field map modelling flow of continuous media and discrete objects. This representation, in contrast to the work presented in the first part of this thesis, does not model occupancy changes directly. Instead, it employs a field of Gaussian Mixture Models, whose local elements are probability distributions of (instantaneous) velocities, to describe motion patterns. Since it assumes only velocity measurements, the proposed representation have been used to model a broad spectrum of dynamics including motion patterns of people and airflow. Using a Gaussian Mixture Model allows to capture the multimodal character of real-world dynamics (e.g. intersecting flows) and also to account for flow variability. In addition to the basic learning algorithms, I present solutions (sampling-based and kernel-based approach) for the problem of building a dense Circular Linear Flow Field map using spatially sparse but temporally dense sets of measurements. In the end, I present how to use the Circular Linear Flow Field map in motion planning to achieve flow compliant trajectories. The capabilities of Circular Linear Flow Field maps are presented and evaluated using simulated and real-world datasets.

The spectrum of applications for the representations and approaches presented in this thesis is very broad. Among others, the results of this thesis can be used by service robots providing help for passengers in crowded airports or drones surveying landfills to detect leakages of greenhouse gases. In the case of a service robot interacting with passengers in a populated airport, the information about the flow of passengers allows to build not only the shortest path between points "A" and "B" but also enables the robot to behave seamlessly, unobtrusively and safely. In the case of a drone patrolling a landfill the impact of airflow, is equally significant. In this scenario, information about airflow allows harnessing the energy of airstreams to lower the energy consumption of a drone. Another way to utilise information about the wind flow is to use it to improve localisation of sources of gas leakage. 



\title{
Podziękowania
}

\author{
(...) żeby napisać jedno własne zdanie, \\ trzeba przeczytać tysiace cudzych. \\ - Ryszard Kapuściński
}

Kiedy byłem nastolatkiem, na festiwalu nauki we Wrocławiu, usłyszałem wykład o autonomicznym robocie szukającym źródeł promieniowania jonizującego. Ten właśnie wykład pchnął mnie na ścieżkę w stronę doktoratu. Dlatego moje pierwsze podziękowanie kieruję w stronę tego, dziś już anonimowego dla mnie, wykładowcy, który nieświadomie zmienił moje życie.

Największe podziękowania składam: prof. Achimowi Lilienthalowi, dr Martinowi Magnussonowi oraz dr Jariemu Saarinenowi, którzy wzięli mnie pod swoje skrzydła i podjęli się pokierowania moimi pierwszymi krokami na naukowej drodze. Prof. Lilienthalowi dziękuję za zaufanie, jakim mnie obdarzył. Dr Magnussonowi jestem wdzięczny za nieustające wsparcie i wysłuchiwanie wszelkich pomysłów. Dr Sarinenowi dziękuję za inspiracje. W tym miejscu pragnę również podziękować dr Januszowi Jakubiakowi, który wziął mnie pod swoje skrzydła w czasie pracy nad moją pracą inżynierską i magisterską.

Niemniej jednak wyrazy mojej głębokiej wdzięczności należą się nie tylko moim promotorom, ale również wszystkim przyjaznym duszom z Uniwersytetu w Örebro. Dr Todorowi Stoyanowowi za nieustającą pomoc w rozwiązywaniu większych i mniejszych problemów z oprogramowaniem, długie i owocne dyskusje o moich pomysłach, jak i za liczne, wspólne podróże. Dr Erikowi Schaffernichtowi za wprowadzenie mnie w świat uczenia maszynowego. Dr Victorowi Hernandezowi Bennettsowi dziękuję za wspólną pracę nad budowaniem map strumieni powietrza.

Dużą część mojego doktoratu stanowiła praca w ramach projektu SPENCER. Wspomnienia z tego projektu zostaną ze mną na długie lata. Szczególne zás miejsce w mojej pamięci zajmują Luigi Palmieri i Timm Linder. Serdecznie im dziękuję za wspólną, nocną zmianę na lotnisku.

Żadnej drogi nie pokonuje się w samotności. W tym miejscu szczególnie chciałem podziękować dwojgu towarzyszy mojej podróży: dr Iran Mansouri i Štefanowi Konečnemu, przez długi czas znajdowaliśmy się w tym samym 
punkcie i ich zrozumienie i wsparcie było nieocenione. Ponadto chcę również podziękować Iran za niezliczone dyskusje na tematy sięgające daleko poza granice codzienności doktoratów, dzięki którym spojrzałem na świat szerzej i głębiej. Ponadto chciałbym podziękować: Uwemu, Lii, Mathiasowi, Marcello, Fabienowi i Jenifer za pomoc w utrzymaniu równowagi między pracą a rozrywką. Chcę również podziękować Ewelinie i Gosi za przyjacielskie wsparcie i niekończące się rozmowy.

Szczególne podziękowania kieruję do moich rodziców: Bożeny i Andrzeja Kucner. To oni zaszczepili we mnie wszystko co dobre i nauczyli mnie jak żyć. To oni natchnęli mnie pasją do nauki i nieustającego rozwoju. To na ich wsparcie zawsze mogłem i mogę liczyć. Pragnę w tym miejscu również podziękować mojej siostrze, Dominice Kucner, która miała niemały wpływ na kształtowanie się mojego charakteru.

Na zakończenie chcę podziękować Matyldzie Czypickiej. To ona pomogła mi przetrwać trudny czas pisania doktoratu, pomogła uwierzyć w siebie i dotrzeć do celu. To dzięki niej trudne chwile mijały szybciej, a dobre trwały dłużej. To ona nadała mojemu życiu ciepłych barw.

Do tej pory udało mi się podziękować z imienia i nazwiska tylko grupce osób. Tym którzy wywarli na mnie największy wpływ w tym właśnie kończącym się okresie życia. Jednak to nie tylko im należy się podziękowanie. W tym miejscu pragnę podziękować wszystkim ludziom, których spotkałem na swojej drodze. To dzięki Waszej pomocy wzrastałem, rozwijałem się, aż dotarłem do tego punktu, w którym się znajduję. Dziękuję Wam wszystkim, bo bez Waszego wkładu nie byłbym tym, kim teraz jestem." 
There are two hard problems in in computer science: cache invalidation, naming things, and off-by-one errors.

Eric Florenzano \& Phil Carlton 



\section{Contents}

1 Introduction 1

1.1 Motivation ...................... 1

1.2 Challenges of Mapping of Dynamics . . . . . . . . . . 2

1.3 Research Question and Contributions . . . . . . . . . . 5

1.4 Publications .................. 7

1.4.1 Included in the dissertation . . . . . . . . 7

1.4.2 Not Included in the dissertation . . . . . . . . . . . 7

1.5 Ethical Considerations . . . . . . . . . . . . . . 10

2 Maps of Dynamics 11

2.1 Detailed Problem Statement . . . . . . . . . . . . . . . . 11

2.1.1 Dynamics perception . . . . . . . . . . . . . 12

2.1.2 Dynamics Categorisation . . . . . . . . . . . . . . 12

2.1.3 Types of Maps of Dynamics . . . . . . . . . . . . . . . 14

2.2 Related Work . . . . . . . . . . . . . . . . . . 15

2.2.1 Mapping Of Spatial Configuration Changes . . . . . . 15

2.2.2 Wind Flow Modelling with Velocity Mapping . . . . . . 21

2.2 .3 Trajectory Mapping . . . . . . . . . . . 22

3 Modelling Motion Patterns with CT-map 25

3.1 Introduction . . . . . . . . . . . . . . 25

3.2 Dynamics Extraction for T-CT-map and CT-map . . . . . . . . 27

3.2 .1 Assumptions . . . . . . . . . . . . 27

3.2.2 Data Pre-Processing . . . . . . . . . . . . . 28

3.2.3 Occupancy Transition Detection . . . . . . . . . . . 28

3.2 .4 Transitions . . . . . . . . . . . . . 35

3.3 The Representations of Dynamics . . . . . . . . . . . . . 37

3.3.1 Conditional Models . . . . . . . . . . . . . . . 37

3.3.2 Parameter Learning . . . . . . . . . . . . . . . . . 39

3.3.3 Conditional Probability Propagation Tree . . . . . . . 41

3.4 Mapping Results . . . . . . . . . . . . . . . . . . . 42 
3.4 .1 Toy Example . . . . . . . . . . . . . . . . . . . 42

3.4 .2 Mapping with CT-map . . . . . . . . . . . . . 46

3.4 .3 Mapping with T-CT-map . . . . . . . . . . . 50

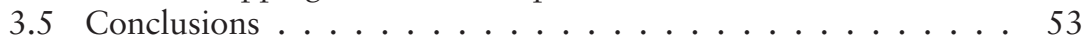

3.5 .1 Contributions ............... 53

3.5.2 Limitations and Future Work . . . . . . . . . 54

4 Modelling Motion Patterns with CLiFF-map 57

4.1 Introduction . . . . . . . . . . . . . 57

4.2 Representation . . . . . . . . . . . . . . . . 59

4.2 .1 Velocity . . . . . . . . . . . . . . . . 59

4.2.2 Semi-Wrapped Normal Distribution . . . . . . . . . . . 60

4.2.3 Semi-Wrapped Gaussian Mixture Model . . . . . . . . 61

4.2.4 Motion Ratio and Observation Ratio . . . . . . . . . . 61

4.3 Map Building . . . . . . . . . . . . . . . . . 63

4.3.1 Data Discretisation . . . . . . . . . . . . . . 63

4.3.2 Mathematical Operations in Circular-Linear Space . . . 63

4.3 .3 Clustering . . . . . . . . . . . . . . . 66

4.3.4 Fitting with Expectation Maximisation algorithm . . . . 69

4.3.5 Ridgeline analysis . . . . . . . . . . . . . . . 70

4.4 Map Densification . . . . . . . . . . . . . . . . . 72

4.4.1 Monte Carlo Imputation . . . . . . . . . . . . . . . 74

4.4.2 Nadaraya Watson Imputation . . . . . . . . . . . 75

4.4.3 Trust Estimation . . . . . . . . . . . . . . . 75

4.5 Evaluation Methodology . . . . . . . . . . . . . 78

4.6 Evaluation . . . . . . . . . . . . . . . . 80

4.6.1 CLiFF-map's Toy Examples . . . . . . . . . . . 80

4.6 .2 Evaluation of Mapping . . . . . . . . . . . . . . 82

4.6.3 Evaluation of Densification . . . . . . . . . . . 94

4.6.4 Guidelines for CLiFF-map Building . . . . . . . . . . 101

4.7 Conclusions . . . . . . . . . . . . . . . . . . . . . . . 101

4.7 .1 Summary . . . . . . . . . . . . . . . . 101

4.7.2 Limitations . . . . . . . . . . . . . . . . 102

4.7.3 Future Work . . . . . . . . . . . . . . . 103

5 Application of CLiFF-map in Motion Planning 107

5.1 Background . . . . . . . . . . . . . . 107

5.1.1 Kinodynamic Motion Planning . . . . . . . . . . 107

5.1.2 Planning in Vector Fields . . . . . . . . . . . . . 108

5.2 CLiFF-RRT* . . . . . . . . . . . . . . . . . . 109

5.2.1 The Algorithm . . . . . . . . . . . . . . . . 109

5.2.2 Extended Upstream Criterion . . . . . . . . . . . . 112

5.2 .3 Steer Function . . . . . . . . . . . . . . . . . . . . 112

5.2 .4 Algorithm Properties . . . . . . . . . . . . . . . 113 
5.3 Evaluation . . . . . . . . . . . . . . . . . . . . 114 5.3 .1 Results . . . . . . . . . . . . . . . 116

5.4 Conclusions . . . . . . . . . . . . . . . . 117

6 Closing Remarks 119

6.1 Contributions . . . . . . . . . . . . . . . . 119

6.1.1 CT-map and T-CT-map . . . . . . . . . . . . . 119

6.1 .2 CLiFF-map . . . . . . . . . . . . . 120

6.2 Limitations . . . . . . . . . . . . . . . . . . . 121

6.3 Future Research Directions . . . . . . . . . . . . . . . . 122

6.3.1 Representations Developments . . . . . . . . . 122

6.3.2 Prospective Applications . . . . . . . . . . . . . . 124

References 



\section{List of Figures}

1.1 A robot in a busy airport - motivating example. . . . . . . . . 3

1.2 Impact of map of dynamics on robot's trajectory. . . . . . . . 4

1.3 Drone path used during data collection. . . . . . . . . . 6

2.1 Dynamics categorisation. . . . . . . . . . . . . . . . 14

3.1 Mapping with CT-map and T-CT-map. . . . . . . . . . 26

3.2 Occupancy shift through $1 \mathrm{D}$ environment. . . . . . . . . . 29

3.3 Roundabout in front of Örebro University. . . . . . . . . . 30

3.4 Intervals separation. . . . . . . . . . . . . 31

3.5 Example of cross-correlation interval separation. . . . . . . . 33

3.6 Cross-correlation plot. . . . . . . . . . . . . . . . . . 34

3.7 Intersection example. . . . . . . . . . . . . . . . 36

3.8 Parameters of T-CT-map. . . . . . . . . . . . . . . 38

3.9 Construction of a CPP-tree. . . . . . . . . . . . . . . 42

3.10 A toy example setup for CT-map and T-CT-map. . . . . . . . . 43

3.11 A toy example of Temporal Conditional-Transition Map. . . . . 44

3.12 Gaussians representing different bivariate distributions. . . . . . 45

3.13 A visualisation of CT-map for a roundabout. . . . . . . . . . 47

3.14 Progress in mapping process. . . . . . . . . . . . . . . . . 48

3.15 CPP-tree for a roundabout data set. . . . . . . . . . . . . . . 49

3.16 Edinburgh test environment. . . . . . . . . . . . . . . 50

3.17 A visualisation of T-CT-map for Edinburgh dataset. . . . . . . . 51

3.18 Comparison of motion patterns depending on entry shifts. . . . 52

4.1 Mapping with CLiFF-map. . . . . . . . . . . . 58

4.2 An example of SWGMM wrapped on a unit cylinder. . . . . . . 62

4.3 Measurement discretisation procedure. . . . . . . . . . . 64

4.4 Comparison of different distance metrics. . . . . . . . . . . 65

4.5 Example of clustering with OPTICS. . . . . . . . . . . . . 69

4.6 Example of Ridgeline analysis. . . . . . . . . . . . . . 71 
4.7 Jonson data collection trajectory. . . . . . . . . . . . 73

4.8 Comparison of imputation methods. . . . . . . . . . . . . 74

4.9 GP representing trust factor - complete observations . . . . . . 77

4.10 GP representing confidence factor - partial observations . . . . 78

4.11 CLiFF-map addressing multiple modes. . . . . . . . . . . . 81

4.12 Example of Edinburgh motion pattern . . . . . . . . . 83

4.13 Input velocity data. . . . . . . . . . . . . . . . . . . 84

4.14 Examples of initialisation with different clustering methods. . . 85

4.15 SWGMM for different initialisations. . . . . . . . . . . 86

4.16 Evaluation results for Edinburgh pedestrian data. . . . . . . . 87

4.17 Visualisation of drone data. . . . . . . . . . . . . . . . 90

4.18 Evaluation results for Drone data. . . . . . . . . . . . . 92

4.19 SWGMM obtained with different initialisation. . . . . . . . . 93

4.20 Sparse densification from 221 locations. . . . . . . . . . 95

4.21 Sparse desification from 20 locations. . . . . . . . . . . 95

4.22 Analysis of mapping. . . . . . . . . . . . . . . . 96

4.23 Analysis of densification with Monte Carlo imputation. . . . . . 96

4.24 Analysis of densification with Nadaraya Watson imputation. . . 97

4.25 The box-plot of divergence for dense maps . . . . . . . . . 97

4.26 Densification quality with respect to kernel size. . . . . . . . . . 99

4.27 Monte Carlo densification Foundry data set. . . . . . . . . . . 100

4.28 Nadaraya Watson densification Foundry data set. . . . . . . . 100

4.29 Example of graph used for distance computation. . . . . . . . . 104

4.31 Comparison of densification of flow map. . . . . . . . . 106

5.1 A result of a planning approach over CLiFF-map . . . . . . 108

5.2 Example smooth paths generated by the POSQ steer function. . 113

5.3 Paths for $P$ and $L$ environment. . . . . . . . . . . . . . . . 115

5.4 Path for a maze environment. . . . . . . . . . . . . . . . 115

5.5 Cost convergence plot. . . . . . . . . . . . . . . . 116 


\section{List of Tables}

4.1 Numerical results for quality of mapping estimation for Edinburgh pedestrian data. . . . . . . . . . . 88

4.2 Numerical results for quality of mapping estimation for drone data set. . . . . . . . . . . . . . . . . . . 91

4.3 Dispersion comparison. . . . . . . . . . . . . . . . . 93

4.4 Baseline comparison . . . . . . . . . . . . . . . . 98

5.1 Experimental results: Planning times of Dijkstra . . . . . . . 116

5.2 Experimental results: Trajectory quality and planning efficiency for $L$ environment . . . . . . . . . . . . . . . 117

5.3 Experimental results: Trajectory quality and planning efficiency for Maze environment . . . . . . . . . . . . . . . 117

5.4 Experimental results: Trajectory quality and planning efficiency for $P$ environment . . . . . . . . . . . . . . . . . . . . 118

5.5 Experimental results: Trajectory quality and planning efficiency for Intersect environment ． . . . . . . . . . . . . . . 118 



\section{Chapter 1 Introduction}

\subsection{Motivation}

Heraclitus of Ephesus (c. 535 - c. 475 B.C.) was one of the natural philosophers, people who have rejected the theological explanation of the world and sought logical explanations for phenomena. He explicitly stated that an inherent feature of the world is motion, or as Platon quotes him in Cratylus: "Everything changes and nothing remains still(...)" This statement is equally true for discrete objects, such as people, and continuous media, like air.

Even though, the assertion of Heraclitus of Ephesus is more than 2500 years old, in many areas of robotics, until recently dynamics was considered primarily as a distortion. However, we can now observe an increasing interest in the problem of modelling dynamics (e.g. learning patterns of motion in the environment). From Human-Robot Interaction (HRI) to Mobile Robot Olfaction (MRO) problems, many research domains in robotics already benefit from maps that go beyond mere occupancy and add an explicit model of dynamics.

In the field of HRI, maps describing dynamics of people provide crucial information for navigation in populated environments [37]. Information about motion patterns in an environment can be helpful in planning socially compliant trajectories, avoiding congested areas and following the general flow of people. This may help the robot to operate unobtrusively [60]. The improvement in planning and navigation will not only result in higher and more human-friendly performance but also can increase safety while executing tasks. Safety and human-friendly behaviour are of vital importance for robotic systems working in the vicinity of people.

Airport Scenario Let us consider the case of a robot travelling through an airport. In Fig. 1.1 we can see an instance of this problem. The robot should travel to the opposite end of a long and crowded airport corridor. We require from the robot to reach the destination fast, safely and in a socially acceptable way. To reach the destination fast, the robot should follow the path corresponding 
to the shortest time of travel. To reach the destination safely, the robot should avoid collisions with static and dynamic obstacles. To move in a socially acceptable way the robot should follow a set of social norms. Work presented in the thesis is concerned with mapping dynamics so as to enable socially compliant behaviour generation by allowing robots to prefer paths that are not in conflict with the observed flow patterns in the environment. I also present how to use such maps with a motion planner and discuss the resulting trajectories.

In the case shown in Fig. 1.1, it is evident that without the information about the general flow of people the robot would still be able to reach the other end of the corridor successfully (see Fig. 1.2). However, it would be more likely to collide with people, might have been delayed by congestion or annoy travellers by moving against the flow. To obtain a map that represents the general flow of people we can use information collected for example with a robot's people tracker during previous missions.

Wind Mapping Scenario In the field of MRO and environmental monitoring, information about air-flow is very important. Let us consider a second scenario in which an autonomous drone is patrolling a landfill site to detect leaks of methane. In this case, airflow is an essential factor influencing at least two aspects of the patrolling mission: trajectory planning and gas source localisation. In the process of motion planning, information about the general air flow is useful to design trajectories which use the wind to extend the distance travelled with one battery charging. In case of gas source localisation, airflow information is essential since advection (movement of molecules caused by fluid flow) is the key gas dispersal mechanism. The importance of airflow information is reflected in a family of bio-inspired algorithms based on anemotaxis, which means that there is a mechanism in which the movement of the robot is determined by the sensed airflow. Although this has not been explored much in the MRO community, it is intuitively clear that a map of the airflow obtained during previous patrolling missions might provide beneficial information for gas source localisation.

\subsection{Challenges of Mapping of Dynamics}

Mapping of dynamics is challenging for many reasons ranging from noisy measurements to the question how to best represent Maps of Dynamics (MoDs). In particular, we can enumerate three primary challenges to be addressed: data stochasticity, partial observability and representation design.

Data Stochasticity Data Stochasticity refers to two issues affecting the measurement process: observation noise and randomness of the process generating the observations. Observation noise is a common issue for all perception processes; it is caused in part by faulty sensors or sensing methodologies. In most 


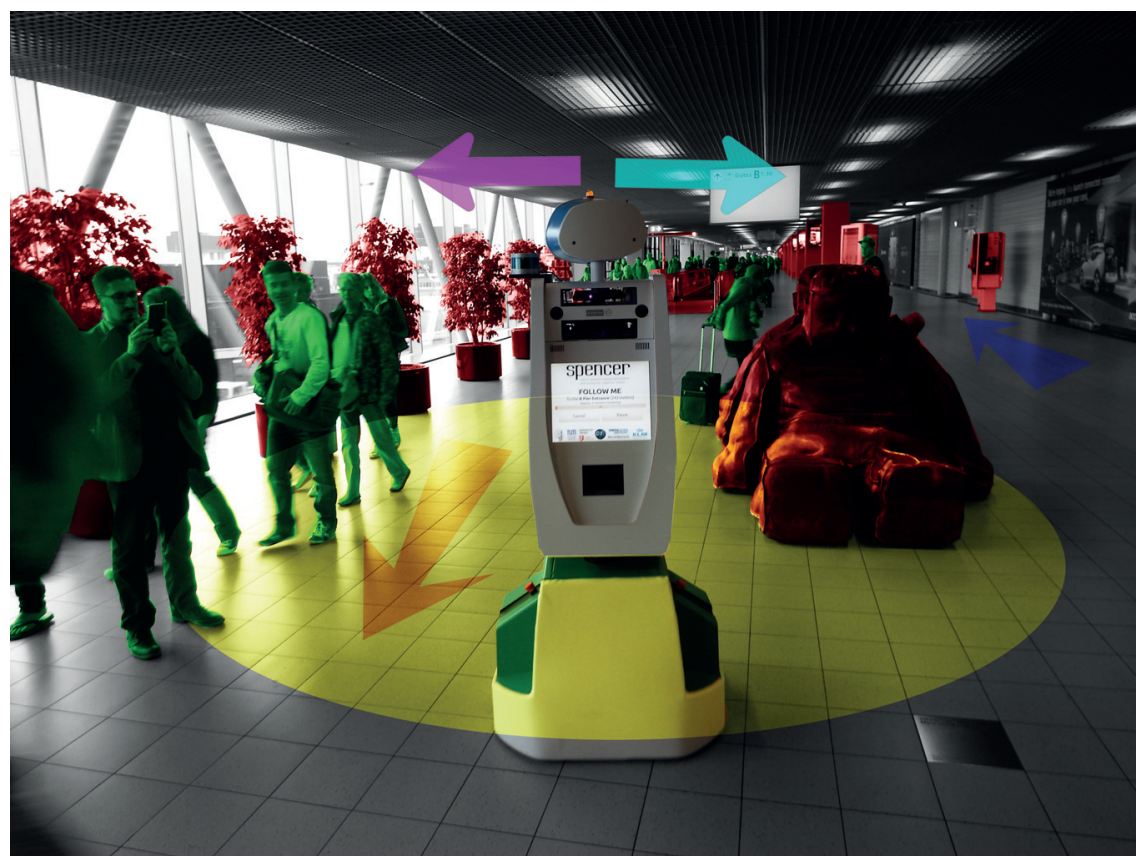

Figure 1.1: In the scenario is shown, a robot has to reach the other end of a long and crowded airport corridor. The robot can either go on the right side of the statue (cyan arrow) or the left (magenta arrow). In the planning process, the robot will have to consider static obstacles (marked red), dynamic obstacles (marked green) in the range of the sensor (visualised by a yellow area) and the flow information (red and blue arrow). Faced with all this information, we would like the robot to choose the path to the right to follow the usual flow of people.

cases, observation noise can be addressed during post-processing of raw measurements (e.g. by a filtering procedure) or in a data integration step (e.g. if the measurement is accompanied with information about uncertainty), and the impact on the MoD of such noise can be mitigated.

The work presented in the thesis relies on the assumption that dynamics within the environment follow some pattern. This pattern can be obscured by a noise coming either from observations or by disturbances affecting the process itself (e.g. traffic affects a commuter bus schedule).

Because patterns modelled with $\mathrm{MoD}$ are affected by noise, an algorithm used to build MoD should be able on the one hand to extract the information about the underlying pattern from noisy data and on the other hand, provide information regarding the variability of the pattern. 


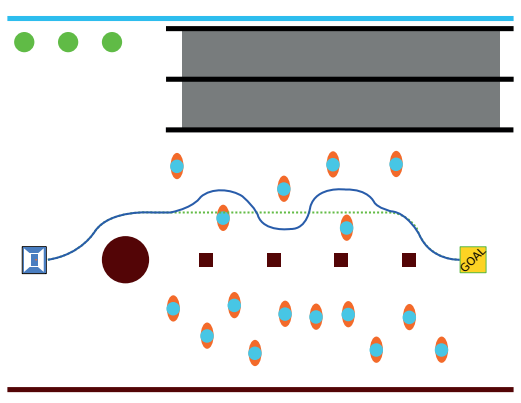

(a) Robot's path in a case when only information about the intensity of dynamics is available.

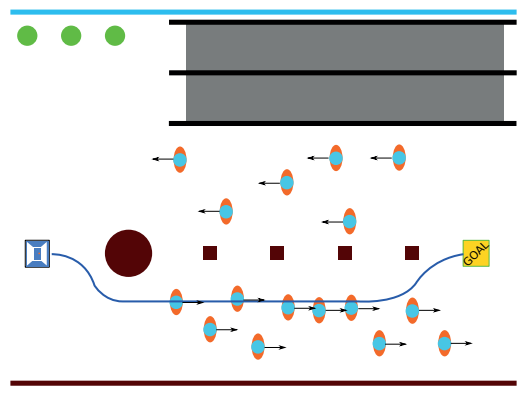

(b) Robot's path in case when also information about the flow of people is available.

Figure 1.2: A simplified top view of the situation presented in Fig. 1.1. The robot (blue and white square) has to reach a location on the far end of the corridor (yellow square with word "GOAL" inside). The robot should avoid people (orange-blue markers) and obstacles (brown markers correspond to columns and sculpture, the green ones to plants and the black-grey structure depict conveyer belts). Fig. (a) shows a situation where the robot plans to go on the left side where the intensity of motion is lower. However, because people are going in the opposite direction, the robot has to execute multiple avoidance manoeuvres. Fig. (n) shows a situation where the robot has access to information about the direction of flow. In this case, the robot is taking a route on the right. In this case, even though the intensity of dynamics is higher, the robot follows the flow and does not have to execute any avoidance manoeuvre.

Partial Observability The impact of the problem of partial observability is substantially greater in the context of MoDs than on static maps. In a static environment, a robot can travel through the environment and extend a static map without risk of losing information. However, if the goal of it is to build a MoD, data ideally need to be collected from the whole environment simultaneously. Unfortunately, in the majority of real-world scenarios, the environment cannot be observed by a single sensor; either because the size of the environment is larger than the sensor range or because of obstacles that occlude parts of the environment. One way to tackle this problem is to build a sensor network covering the whole area of interest. Unfortunately, for many real-life applications, building a dense sensor network is unfeasible. It is often more efficient to equip a mobile platform with sensors and patrol a region of interest. This setup tends to produce data that are spatially denser but temporally sparser than data from a stationary sensor network. The issue of partial observability affects both observations of the motion of discrete objects and continuous media. Below, I 
describe the ways partial observability affects the two scenarios presented in this chapter.

Airport scenario Here, the goal is to model the motion of discrete objects, and the robot is equipped with a range sensor of a limited range (see Fig. 1.1), additionally obstacles and moving objects causes multiple occlusions. As a result, the robot can only observe fragments of the environment at once.

Wind mapping scenario In the case of continuous media, the problem of partial observability is also present. In Fig. 1.3 we can see the trajectory of a microdrone while collecting wind measurements. In this scenario, the wind measurements can be obtained only at the exact locations the robot visited and only for the period when the robot was present at a location.

Representation Design In the process of representation design it is necessary to answer following three questions:

1. What kind of information can be used for building the representation?

2. What is the representation for?[21]

3. How close is the representation to the real thing?[21]

These three questions define three problems to be addressed in the process of representation design.

The first one refers to the problem of data availability. In the process of representation design, it is necessary to account for the quality of the available data. For instance in the airport scenario, where it is almost impossible to collect complete tracks of people, building track dependent trajectories would be unfeasible.

The second question encapsulates the problem of usability of the representation. The design and choice of representation heavily depend on the application; the map should represent the data in a convenient way for other modules of the system.

Finally, the third question relates to the problem of precision of the representation. In other words, how detailed the representation should be to maintain useful information but not to model noise.

\subsection{Research Question and Contributions}

The work presented in this thesis was conducted in an attempt to answer the following research question:

How to map motion patterns, from incomplete and noisy data, for mobile robots? 


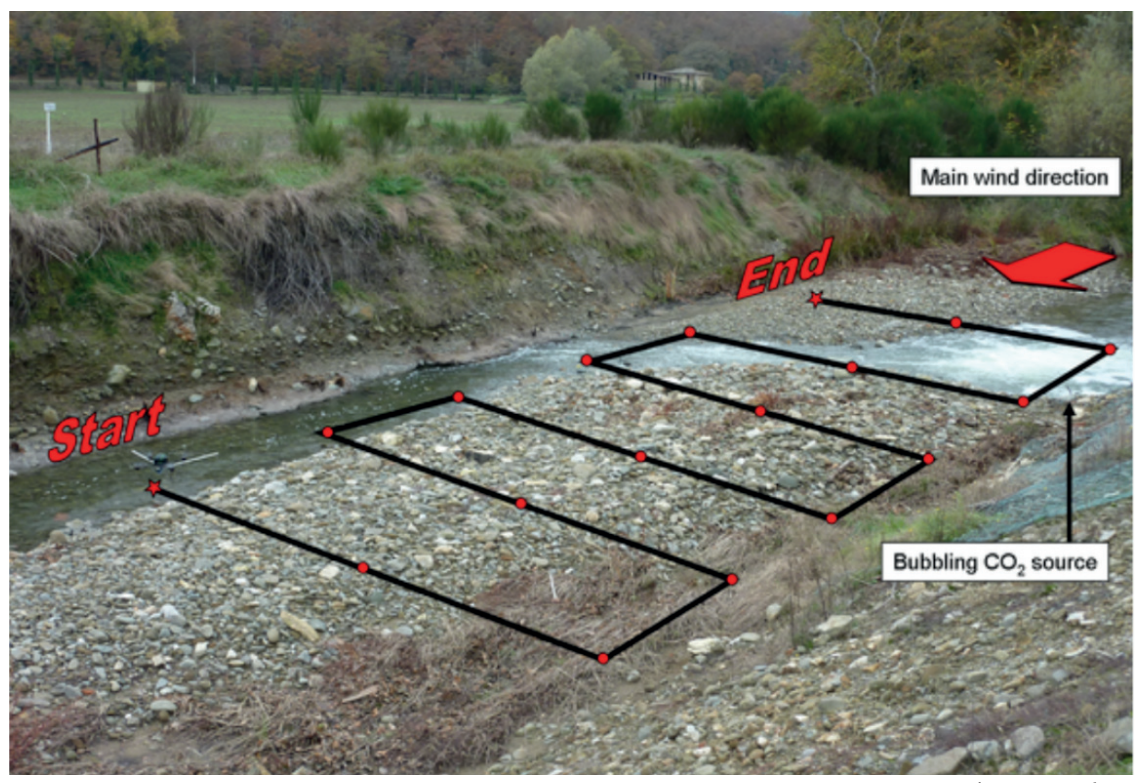

By courtesy of: Neumann et al. [53]

Figure 1.3: Preprogrammed path followed by a drone during collection of wind measurements.

In an attempt to answer this question I provide the following contributions:

- I introduce a grid based representation called Conditional-Transition Map (CT-map), and its extension Temporal Conditional-Transition Map (T-CT-map), that captures the dependency between the occupancy changes in adjacent cells.

Chapter 3

- I introduce a probabilistic representation called Circular Linear Flow Field map (CLiFF-map), which represents a turbulent flow of a continuous medium as well as motion patterns of discrete objects using a set of Semi Wrapped Gaussian Mixture Models (SWGMMs). Chapter 4

- I present methods of building CLiFF-map using spatially sparse but temporally dense data.

Chapter 4

- I present application of the CLiFF-map for social aware motion planning. (a joint work with Luigi Palmieri [60].) Chapter 5 


\subsection{Publications}

\subsubsection{Included in the dissertation}

Parts of this work has previously appeared in the following publications. In all articles for which I am the first author, I have performed the relevant software implementation and tests, as well as the major part of analysing and reporting the obtained results.

- Tomasz Piotr Kucner, Jari Saarinen, Martin Magnusson, and Achim J. Lilienthal. Conditional transition maps: Learning motion patterns in dynamic environments. In IEEE/RSJ International Conference on Intelligent Robots and Systems :, pp. 1196-1201, 2013.

Part of Chapters 2, 3

- Tomasz Piotr Kucner, Martin Magnusson, Erik Schaffernicht, Victor Hernandez Bennetts, and Achim J. Lilienthal. Tell me about dynamics! Mapping velocity fields from sparse samples with Semi-Wrapped Gaussian Mixture Models. In RSS 2016 Workshop: Geometry and Beyond - Representations, Physics, and Scene Understanding for Robotics, 2016.

Part of Chapters 2, 4

- Tomasz Piotr Kucner, Martin Magnusson, Erik Schaffernicht, Victor Hernandez Bennetts, and Achim J. Lilienthal. Enabling flow awareness for mobile robots in partially observable environments. In IEEE Robotics and Automation Letters, vol. 2, no. 2, pp. 1093-1100, 2017.

Part of Chapters 2, 4

- Luigi Palmieri, Tomasz Piotr Kucner, Martin Magnusson, Achim J. Lilienthal, and Kai Oliver Arras. Kinodynamic motion planning on Gaussian mixture fields. In IEEE International Conference on Robotics and Automation (ICRA 2017), 2017.

Part of Chapter 5

(In this joint work, I have contributed to design of a cost function for the motion planner, design of experiments, evaluation and reporting the results.)

\subsubsection{Not Included in the dissertation}

In the time of $\mathrm{PhD}$ studies I have also contributed my work to following publications, which did not become part of my thesis: 
- Rudolph Triebel, Kai Oliver Arras, Rachid Alami, Lucas Beyer, Stefan Breuers, Raja Chatila, Mohamed Chetouani, Daniel Cremers, Vanessa Evers, Michelangelo Fiore, Hayley Hung, Omar A. Islas Ramírez, Michiel Joosse, Harmish Khambhaita, Tomasz Piotr Kucner, Bastian Leibe, Achim J. Lilienthal, Timm Linder, Manja Lohse, Martin Magnusson, Billy Okal, Luigi Palmieri, Umer Rafi, Marieke van Rooij, and Lu Zhang. SPENCER: A Socially Aware Service Robot for Passenger Guidance and Help in Busy Airports. In Field and Service Robotics: Results of the 10th International Conference, vol. 113 in Springer Tracts in Advanced Robotics, pp. 607622, 2016.

(This paper summarises work on a socially compliant mobile robotic platform, which was developed in the EU-funded project SPENCER. The purpose of this robot was to assist, inform and guide passengers in large and busy airports. My involvement in work presented in this paper was two-fold. First, I was responsible for maintaining the mapping and localisation system deployed on the robot. Second, I was working on building maps of dynamics.)

- Tomasz Piotr Kucner, Martin Magnusson, and Achim J. Lilienthal. Where am I? an NDT-based prior for MCL. In 2015 European Conference on Mobile Robots (ECMR) :, IEEE conference proceedings, 2015.

(The contribution of this paper is a method to build a prior distribution of samples for Monte Carlo Localisation.)

- Martin Magnusson, Tomasz Piotr Kucner, and Achim J. Lilienthal. Quantitative evaluation of coarse-to-fine loading strategies for material rehandling. In Proceedings of the IEEE International Conference on Automation Science and Engineering (CASE) :, IEEE International Conference on Automation Science and Engineering (CASE), pp. 450-455, IEEE conference proceedings, 2015.

(The main contributions of the paper is a quantitative and qualitative evaluation of pile handling strategies. Furthermore, the paper also presents a complete methodology for evaluation of pile handling strategies. I have contributed to the development of experimental setup and data collection.)

- Victor Hernandez Bennetts, Erik Schaffernicht, Achim J. Lilienthal, Han Fan, Tomasz Piotr Kucner, Lena Andersson, and Anders Johansson. Towards occupational health improvement in foundries through dense dust and pollution monitoring using a complementary approach with mobile and stationary sensing nodes. In Proceedings of the IEEE/RSJ International Conference on Intelligent Robots and Systems (IROS) :, pp. 131- 
136, 2016.

(This paper presents, a novel heterogeneous system for the task of pollution monitoring in foundries using a mobile robot and a sensor network. In work for this paper, I have contributed to discussions over the problem of building wind maps.)

- Victor Hernandez Bennetts, Tomasz Piotr Kucner, Erik Schaffernicht, Patrick Paul Neumann, Han Fan, and Achim J. Lilienthal. Probabilistic airflow modelling using turbulent and laminar characteristics for ground and aerial robots. IEEE Robotics and Automation Letters, vol. 2, no. 2, pp. 1117-1123, 2017.

(The main contribution of the paper is a method for airflow modelling in microscale environments, distances smaller than $2 \mathrm{~km}$, which considers the laminar and turbulent character of the air flow. In this work I have mostly contributed to discussions about the problem formulation and about the evaluation strategies.)

- Hongqi Fan, Tomasz Piotr Kucner, Martin Magnusson,Tiancheng Li, and Achim J. Lilienthal, A dual PHD filter for effective occupancy filtering in a highly dynamic environment, IEEE transactions on intelligent transportation systems (Print), vol. PP, no. 99, pp. 1-17, 2017.

(This paper focuses on extracting information about the flow of occupancy over a grid map using a dual probability hypothesis density filter. In this work, my work was focused on problem formulation, discussions over the solution, evaluation of designed solution and reporting the results.)

- Håkan Almqvist, Martin Magnusson, Tomasz Piotr Kucner, and Achim J. Lilienthal, Learning to detect misaligned point clouds, Journal of Field Robotics, 2017.

(The contribution of this work is two-fold. First, is the evaluation of existing methods for detecting whether two point clouds are aligned or not. Second, is the usage of boosting to learn a strong classifier by combining the weaker individual classifiers. My contribution focused on implementation of one of the evaluated classifiers and on reporting the results.)

- Martin Magnusson, Tomasz Piotr Kucner, Saeed Gholami Shahbandi, Henrik Andreasson, and Achim J. Lilienthal, Semi-supervised 3D place categorisation by descriptor clustering, in 2017 IEEE/RSJ International Conference on Intelligent Robots and Systems (IROS) :, pp. 620-625, 2017. 
(The main contribution of the paper is to divide scans into semantically meaningful clusters corresponding to different types of environments using existing clustering methods without previous training. In this work, I have contributed to the discussion over the problem, part of the evaluation process and in reporting of the results.)

\subsection{Ethical Considerations}

Since the invention of first stone tools, the technological progress has been bringing boons and courses simultaneously on humankind, despite the intentions of the creators. The work presented in this thesis is also not free from this duality, and only in hindsight, it will be possible to assess the impact of my contribution accurately.

The work presented in this thesis focuses on providing tools to extract information about motion patterns in the environment. Such tools are not dangerous as such, and the possible benefits or hindrances arise only from the ways how these tools are used.

Currently, we are in the midst of the robotic and automatic revolution where autonomous agents are supporting people in their work and daily lives. In many cases, such autonomous agents replace humans in executing tedious or dangerous tasks. It is not yet clear how the future ahead of us look. If it will be a place where people will be leaving happy life focusing on their passions or if we are facing grim time where people will be treated as dispensable. Therefore it is necessary to pay continuous attention if the newly developed tools are used for the benefit of people. 


\section{Chapter 2 Maps of Dynamics}

\subsection{Detailed Problem Statement}

So far I have relied on an intuitive understanding of the concept of a MoD. However, because of domain dependent understanding of terms map and dynamic, it is necessary to define these terms precisely.

A map is a way to encode spatial information; it can be information about the position of obstacles in the environment or locations of gas sources, for example. Similarly, a MoD represents "the dynamics" at different locations.

The understanding of the term dynamics depends on the context in which it is used. In the field of classical mechanics, dynamics is the branch concerned with a study of forces and torques and their effect on motion. A dynamical system in mathematics refers to a system in which a function describes the time dependence of a point in a geometrical space. In computer science, a dynamic data structure refers to an organisation or collection of data in memory that has the flexibility to grow or shrink in size. However, this thesis adopts the term dynamics as it is used in the field of the robotic mapping. Hähnel et al. [29] define that an environment is dynamic if it undergoes changes or moving objects are present therein. This description distinguishes a dynamic environment from a static environment, where neither moving objects nor other visible changes are present. This understanding of dynamics is tightly connected to the motion of discrete objects. The situation differs in some crucial aspects of the motion of microscopic objects, such as air particles, is observed. In contrast to macroscopic objects, tracking each particle of the air is not feasible, and it is reasonable to consider the motion of discrete objects as a flow of a continuous medium. This leads to an extension of the previous understanding of dynamics in the context of robotic mapping. In my thesis, whenever I will be referring to dynamics, I will consider a motion of discrete objects, a flow of continuous media or both. Considering this extended definition of dynamics, this part of my thesis will be dedicated to the following research question: 
How to efficiently map the motion of macroscopic objects (i.e. people) and the flow of a continuous medium?

\subsubsection{Dynamics perception}

The way dynamics are represented in a MoD directly depends on the way dynamic changes are perceived by a robot. I suggest a division into three classes:

Perception of velocity - when dynamics are observed as velocity measurements obtained either with a dedicated sensor (e.g. anemometer) or by estimating it based on the object position change between consecutive observations.

Perception of a trajectory - when dynamics are perceived as a time-stamped series of poses of an object; it is possible to observe full trajectories. Trajectories cannot be observed in the case of the flow of continuous media.

Perception of spatial configuration changes - when velocity measurements are not directly available, but the dynamics can be observed as a difference between subsequent observations of the environment. I will discuss this type of perception only in connection with the flow of macroscopic objects in my thesis.

In the following subsection, I will discuss different types of dynamics and present for each type a corresponding perception method.

\subsubsection{Dynamics Categorisation}

In the beginning of Sec 2.1 I have described two primary classes of dynamics: motion of discrete objects and flow of continuous media. A way to draw a line between these two classes is to distinguish if the dynamic objects are treated independently or en masse.

\section{Flow of Continuous Media}

In cases when the length scale is much greater than the distances between the dynamic objects, it might be more convenient to model large groups of such objects as a continuum. This assumption is often made for microscopic objects, such as particles of air, but can also be applied to macroscopic objects, e.g. people in large groups [79].

The primary advantage of treating dynamics as a flow of continuous medium is the ability to address motion of large groups of objects in a convenient tractable way; however, that also limits the perception capabilities. Since information about separate entities is ignored, it is impossible to obtain information about trajectories of individual objects. The flow (laminar or turbulent) of a continuous medium can be perceived through velocity measurements. 


\section{Motion of Discrete Objects}

In many cases, it is possible and necessary to model the motion of multiple objects as a flow of a continuous medium. However, there is also a large group of tasks where the motion of discrete objects can and should be perceived and modelled independently for each object.

For discrete objects, we can distinguish following classes of dynamics. The most straightforward distinction is between objects that remained static or moved during a robot's mission. The latter class can be sub-divided further as, e.g. a food truck, which moves between key selling locations in the city during the day but for most of the time remains still, versus a car riding on a highway, which can be observed in motion. Meyer-Delius et al. [48] suggested the following classification of dynamics, which I adopt in this thesis:

Static - An object is static if it is observed in the same state through at least a major part of a mission.

Semi-static - An object is considered semi-static if it is often observed by a robot in different but only a few states.

Dynamic - An object is considered dynamic if it is observed by a robot in many different states and can also be observed during the change of its state.

The first notable aspect of this classification is that it refers to the duration of a robot's mission. If a robot is executing a short mission in a parking lot, some cars may belong to the dynamic category while most of the vehicles are static because they will be in the same location at the beginning of the mission and remain in the same position until the end of the mission. However, if the robot is patrolling the same parking lot for several weeks, cars will be rather semi-static or dynamic objects.

A second important aspect deserves to be highlighted: objects can change the category during the execution of a mission, and this happens in particular for semi-static and dynamic objects. The cars in the mentioned parking lot can be perceived as semi-static as long as the robot only sees the changes of their static states or dynamic if the robot observes them moving.

Depending on the class the motion of discrete objects they can be perceived in different ways. Dynamic objects can be perceived in all three ways mentioned above, through velocity measurements, through observations of a trajectory and observations of spatial configuration changes. In the case of semi-static or static objects, we are limited to observations of spatial configuration changes or lack of them.

Fig. 2.1 shows a categorisation of types of dynamics, accompanied by a table listing which observation methods are viable for which types of dynamics. As I have emphasised in this and the previous section not all types of dynamics can be perceived in all possible ways. Moreover, it is important to emphasise that even though a class of dynamics can be perceived with a particular method, 


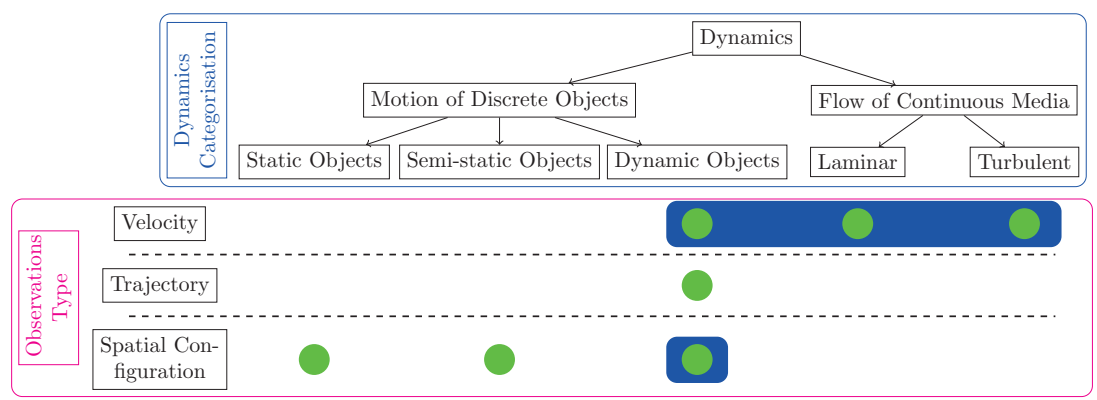

Figure 2.1: The top part of the figure shows categories of dynamics. The bottom part of the figure shows how different types of dynamics can be observed and in result mapped. The green dots mark how different types of dynamics can be perceived. Finally, the solid blue rectangle marks the areas of interest addressed in the thesis.

it does not mean this is always the case. For example in a cluttered environment, it might be difficult to obtain a complete track of a discrete object, and the only reliable information may be velocity measurements or short parts of full tracks.

\subsubsection{Types of Maps of Dynamics}

Few sensors can perceive dynamics directly. For the most part, the perception of dynamics requires the initial stream of raw data to undergo one, or more, processing steps. Therefore the perception of dynamics depends not only on the stream of input data from a sensor but also on the ways the data is further processed. In consequence, the type of $\mathrm{MoD}$ depends on the perception of dynamics, understood in a broad way including both the type of raw data and result of further processing. This phenomenon is especially visible in the context of dynamic objects. As shown in Fig. 2.1, the motion of dynamic objects can be observed and in consequence, mapped in all three possible ways (i.e. velocity mapping, trajectory mapping, mapping of spatial configuration changes). Furthermore, there is a sequential dependency between the perception methods, in the case of dynamic objects. Namely, observation of shape configuration changes can be used as input to an object tracking algorithm and finally the tracks of objects can be used to obtain velocity measurements.

In Fig 2.1, I present three types of dynamics perception which corresponds to three types of MoDs with the same names. MoDs based on spatial configuration changes and MoDs based on trajectories can be used for mapping dynamics of discrete objects. The third category, MoDs based on velocity observations can be used for mapping both discrete objects and continuous media. 
In the thesis, I discuss the problem of building MoDs of flow of continuous media, both turbulent and laminar, and MoD of discrete objects. I focus on velocity mapping and mapping of spatial configuration changes. The reason for this choice is that velocity mapping and mapping of spatial configuration changes are more robust against noisy and incomplete observations.

To better understand the vulnerability of trajectory-based mapping let us analyse the airport scenario. In the airport scenario a robot might be equipped with a laser sensor providing a stream of scans depicting the state of the observed environment. The stream of scans can be used by a people tracking system, providing tracks. However, because of a large number of occlusions, disturbing the tracking process, the people tracking system may provide incomplete trajectories (tracklets). Building a map out of tracklets is substantially more difficult than when using complete trajectories, or even impossible. However, tracklets can carry enough information (consecutive time-stamped positions) to estimate velocities and build a velocity map.

A detailed discussion on pros and cons of different MoDs approaches is presented in the following section.

\subsection{Related Work}

It is rather the norm than an exception that the environment of a robot is changing during operation. Therefore, several mapping approaches were developed by the robotics community, to help robots to act in a dynamic environment. The goal of this section is to summarise existing mapping methods addressing the problem of modelling dynamic aspects of the environment.

\subsubsection{Mapping Of Spatial Configuration Changes}

Work on the mapping of spatial configuration changes can be divided into three major groups:

1. Dynamics Ignorance - In the early works on robotic mapping dominated a belief that only the static parts of the environment are essential for robot's operation and therefore only the static elements should be included in a map. Moreover, these methods relied on a static world assumption, which states the only time-dependent part of the environment is the robot itself. Methods following this paradigm do not explicitly model the dynamics; however, they aim to minimise the impact of noise generated by moving objects. For example, in the classic work of Moravec and Elfes [51] assume a static world; however, by accumulating observations the occupancy associated with dynamic objects can be effectively removed from the map.

2. Dynamics Removal - The static world assumption is limiting and can negatively affect the quality of the resulting map. In "Robotic Mapping: A 
Survey" [71], published in 2002, Sebastian Thrun states: "(...) imagine a robot facing a closed door that previously was modelled as open. Such an observation may be explained by two hypotheses, namely that the door status changed, or that the robot is not where it believes to be." Allowing for the possibility that the door status changed lead to the understanding that the information about dynamics should be explicitly handled in the mapping process. One of the approaches is dynamics removal, where the goal is to discard measurements associated with dynamic objects $[29,73]$.

3. Dynamic Map Update - In parallel to dynamics removal methods $d y$ namic map update approaches emerged. In dynamic map update a map, or a dedicated layer of robot's map, is continuously updated to incorporate the most recent information about the state of the dynamic objects in the environment $[3,13,15,50,77]$.

4. Mapping of Dynamics - Finally, around 2010 methods focusing on building models of dynamics started to emerge. The key contrast to previously existing methods is to not only detect and minimise the impact of dynamic changes but rather to learn the pattern governing changes of the spatial configuration. The dominant paradigm is to build a probabilistic representation modelling the probability of occupancy changes in a given part of an environment $[43,49,66]$. However, another valuable approach is to model the dynamics patterns in a frequency domains and to enhance the predictive capabilities of the model of dynamics [36].

\section{Dynamics Removal}

The core idea behind dynamics removal approaches is to detect dynamics and remove its traces in the measurements.

The works of Wang et al. [73] and Hähnel et al. [29] are examples of approaches towards dynamics removal. Wang et al. [73] propose an object-centric approach, combining detection and tracking of moving objects (DTMO) with simultaneous localisation and mapping (SLAM). The core idea of this work is to build a cyclic dependency between DTMO and SLAM. The information provided by DTMO is used to remove dynamic objects from the scans and in this way to improve registration, while the information provided by SLAM is used to detect new dynamic objects and support the detection step of DTMO. This approach can of course only cope with the dynamics caused by objects that the tracking module can recognise. Even though the object tracking module is an integral part of Wang's approach, this method cannot be included into trajectory mapping methods, because the information obtained with the object tracking module is not retained.

In the work of Hähnel et al. [29], dynamics detection are addressed as an outlier detection problem. Namely, the goal is to estimate the likelihood that 
a given measurement is associated with a dynamic object presence in the environment.

These two methods focus on improving the reliability of maps of static parts of the environment. Such a map offers benefits in connection with navigation tasks (in the sense that the resulting map is reliable, contains only static landmarks, and does not include false obstacles). On the other hand, information about dynamics can also be of value for motion planning tasks, e.g. allowing a planner to account for the changes in the environment.

\section{Dynamic Map Update}

For some applications removing information about dynamics might be beneficial. However, retaining information about the dynamic elements of the environment might substantially enhance the capabilities of robotic systems. This section provides an overview of related work focusing on methods retaining information about the dynamic parts of the environment. The work summarised in this section focuses on building models that are capable of quickly adapting to incoming information about dynamics in the environment. It is important to emphasise that these methods are, still, primarily focusing on building a map of spatial representation albeit in a way that also takes into account that the geometry may change over time. In contrast, in the subsequent section, I will describe work on the mapping of dynamics. The mapping of dynamics goes beyond sheer map update and attempt to model the patterns that dynamics are following in the environment and not the configuration of the environment.

Arbuckle et al. [3] proposed an extension to occupancy grids the Temporal Occupancy Grid (TOG), where the history of the observations is stored in a layered occupancy map. In TOG each layer incorporates a certain amount of measurements up to the most recent ones. Arbuckle et al. [3] use the TOG for classifying the dynamics by matching "patterns" (if long-term map and medium term map are empty, but the short-term map is occupied, then a moving object is observed). The downside of the TOG is that the representation needs to preserve the full history of measurement up to the longest timescale.

Biber and Duckett [13] follow a paradigm, similar to the one of Arbuckle et al. [3], of storing past observations. However, instead of fusing observations into any specific representation, they propose to use robust statistics to continuously estimate the shape of the environment based on stored historical observations. Biber and Duckett [13] propose to maintain a number of sets, each one containing past observations of the environment and refresh these sets in different, regular intervals. In this way, the sets are "forgetting" the past state of the environment at a different rate; this refreshing mechanism loosely resembles humans' forgetting process. The representation proposed by Biber and Duckett [13] uses robust statistics in order to compute the updated state of the environment. The robust statistics (i.e. computation of median of a set of measurements) has a dual advantage. First, even if the set contains outliers, 
they will be suppressed by correct observations. Second, the median relates to actual measurements and does not lead to an introduction of artefacts into the map. However, it comes at the cost of memory that is required to store the past observations.

Another method focusing on storing past observations of the environment was presented by Mitsou and Tzafestas in [50]. They introduced a representation also coined Temporal Occupancy Grid (TempOG), which stores the discrete observations as a time signal for each cell in a grid map. The representation uses a time index access structure, which is a special case of a $\mathrm{B}+$ tree. However, the focus of this work is on storing the history of past observations in an efficient way.

In 2007 Burgard et al. [15] proposed a representation, which attempts to minimise the amount of stored data. They suggest to detect dynamic aspects of the environment and efficiently model them instead of storing a complete or partial history of observations. This work presents an intermediate step between two paradigms, from dynamic map update to mapping of dynamics. Burgard et al. [15] attempt to build a map which can quickly adapt to the environment changes, as all dynamic map update methods do. However, they also utilise the knowledge that some parts of the environment follow some patterns (e.g., doors). They introduce a method for learning semi-static states of the environment. The approach extracts the map batches where the dynamics have been observed during different time intervals and learns the different configurations of the batch. Later on, these batches are utilised to improve the localisation.

The methods presented so far in this section focus on building maps which can quickly adapt to the most recent observations of the environment. These approaches present a significant improvement with respect to Dynamics removal techniques. However, this is only a reactive behaviour, in a quickly changing environment, such an approach might be insufficient. In order to improve operation in a quickly changing environment, it is desired to equip robots with abilities to anticipate future configurations of the environment. The work of Chen et al. [18] and Gindele et al. [28] were focused on providing a tool to predict the future occupancy.

In 2006 Chen et al. [18] published work where a Bayesian Occupancy Filter $(\mathrm{BOF})$ was used in a pre-processing step in an object tracking procedure. $\mathrm{BOF}$ is a Bayesian program using the most recent set of observations to estimate the future occupancy of cells. From the object tracking perspective, such an approach has four advantages: explicit modelling of uncertainty, simplification of a data association problem, removal of object modelling problem and easy parallelisation. From the dynamic map update perspective, there is a dual advantage. First, BOF enriches the occupancy information by adding velocity estimates. Second, the method described by Chen et al. [18] allows estimating the future occupancy of the environment. However, this method does not ac- 
count for the shape of the environment neither for existing motion patterns. Therefore the longer the prediction is, the less reliable it is.

Gindele et al. [28] proposed an improvement of the method of Chen et al. [18] by incorporating information about the shape of the environment. However, the method was still relying only on the most recent observations and did not attempt to capture the model of the dynamics of the environment.

\section{Mapping of Dynamics}

Around 2010 a new paradigm emerged; namely, the goal became not only to adapt the spatial representation according to observed changes but also to learn the patterns governing the dynamics. In this section, I present methods following this paradigm in the context of occupancy grid maps.

The methods focusing on mapping of dynamics in occupancy grid maps can be divided into two types. The type 1 methods rely on an independent cell assumption, in other words, the change of a cell's state does not depend on the change of the state of the cell's neighbours. The type 2 methods relax the independent cell assumption. These methods include not only the previous state(s) of the cell but also the impact of adjacent cells.

Type 1 In his $\mathrm{PhD}$ thesis, Meyer-Delius [49] introduces a generalisation of a standard occupancy grid called dynamic occupancy grid. In his work, he models the probability of a cell occupancy as a hidden Markov model and introduces an expectation-maximisation-based approach to learn state-transition probabilities for an occupancy map. The changes in the environment are assumed to be caused by semi-static objects and due to a stationary process.

Saarinen et al. [66] also present a grid-based representation of dynamics, which also assumes cell independence. This work models the probability of state change for each cell as an independent two-state Markov chain (iMac). However, in contrast to the work of Meyer-Delius [49], the model can capture not only semi-static aspects of the environment but also dynamic ones.

Part of type 1 methods focus on modelling occupancy changes; however, dynamics can be described in multiple ways. Luber et al. [43] proposed a multilayer map where each of the layers models a different aspect of the environment dynamics, e.g. probability of appearance of new dynamic objects or areas of high probability of motion. To model such events Luber et al. [43] use a piece-wise homogeneous Poisson process. In other words, to each cell in the grid, there is associated a homogeneous Poisson process with a fixed rate over time, modelling the layer's events.

The methods of type 1 presented so far in this section have limited prediction capabilities and focus only on the probability of the next state change. However, it is reasonable to assume that some of these changes have a periodic character and it should be possible to build a time-dependent model able to perform predictions for a long time horizon. Starting from this assumption, 
Krajnik et al. [36] introduce a representation, which describes a time-dependent probability signal in frequency domain. In such a way, the authors not only substantially compress the stored data but also enables predictive capabilities of the model. Moreover, the model presented by Krajnik et al. [36] is not limited only to represent the occupancy changes in the map but is well suited to model the probability of any binary event (e.g., state of a door, light on or off).

Type 2 Methods of type 2 relax the cell independent assumption. In 2013 I presented one of the first attempts to model the interaction between adjacent cells of a map called CT-map [38]. CT-map associates to each cell a set of conditional distributions, which model the probability of occupancy shift over the cell edge. A detailed description of the method will be presented in chapter 3 .

Furthermore in 2014 Wang et al. [76] proposed a method, which models the interaction between adjacent cells as an input-output hidden Markov model (IOHMM). In this approach, the state of the cell does not only depend on the previous state of the cell and the transition probability in the model but also incorporates external information coming from the adjacent cells. Wang et al. extended their work, and in 2015 they presented Multi-scale conditional transition map [75]. In this work, the IOHMM is not only affected by adjacent cells but also with distant semantically significant locations such as sinks and sources or stop points. This combination allows obtaining long-distance predictions.

The works of Wang et al. [75, 76] emphasises the same concept, as CT-map (presented in chapter 3): that the occupancy changes of a cell depend on the state of its neighbours. However, the primary difference is, which cells are considered as adjacent; Wang et al. [75, 76] only the cells sharing an edge are considered as an adjacent cell. In such a case the diagonal motion of objects might not be properly modelled. In contrast, in CT-map also cells sharing vertices are considered adjacent. Furthermore, the proposed IOHMM is trained with the EM algorithm with all the available data at once; this makes it more difficult to update the map unless all the parameters are recomputed. Moreover, in contrast to the work presented in chapter 3, Wang et al. jointly model the probability of occupancy shift with the time required to shift.

\section{Limitations of Mapping of Spatial Configuration Changes}

The methods summarised in this section are the core of mapping of spatial configuration changes. The work presented in chapter 3 is directly related to these methods. In my work on the mapping of spatial configuration changes, I focus on relaxing the assumption of cell independence (type 1 methods).

However, mapping of spatial configuration changes is not always feasible mapping method (e.g. for wind mapping). Considering, this assertion in chapter $4 \mathrm{I}$ focus on representation directly modelling velocity. Therefore, in order to bring a broader look at the problem of $\mathrm{MoD}$ and provide context for work 
presented in chapter 4 I will present in following sections velocity and trajectory mapping.

\subsubsection{Wind Flow Modelling with Velocity Mapping}

The methods presented so far all work on occupancy grid maps, but some types of dynamics cannot be represented by occupancy maps: e.g., wind flow. Using maps of velocities instead of occupancy makes it possible to represent a flow of wind as well as the flow of objects or people. Even though velocity mapping can be applied for modelling various types of dynamics, it has not received a lot of attention in the robotics community. However, in the field of MRO, velocity mapping has been used in a few applications. For instance, Reggente and Lilienthal [62] show how wind information affects the gas distribution mapping process. However, in their work, the wind information was used in the process of building gas distribution not to build a wind map itself.

In principle the algorithms addressing the problem of wind mapping can be divided into three classes based on the scale of the addressed area: macro (distance up to $5000 \mathrm{~km}$ ), meso (distances up to $200 \mathrm{~km}$ ) and micro (distances below $2 \mathrm{~km}$ ) scale. Most Air Flow Mapping (AFM) methods are concentrated around macro/meso scales with low temporal resolution (e.g. months, days), using weather stations [6]. In micro-scales, simple AFM approaches include the use of trace gases [33], or the use of sophisticated Computational Fluid Dynamics (CFD) simulations as shown by Cao et al. [16]. These methods focus on building weather models which provides wind estimates in a given location at a requested moment in time. These methods are not velocity maps in a strict sense. Moreover, these methods are not directly applicable to mobile robotics. For example, CFD-based techniques require precise a priori knowledge about boundary conditions and these techniques require high computational resources, which may not be available on board of mobile platforms.

From the perspective of a robotic community, air-flow is normally considered as a disturbance to the control problem and has seldom been explicitly modelled. To my best knowledge, there are only a few works that explicitly address the problem of AFM in the context of mobile robotics. In work of Kowadlo et al. [35] a naive physics airflow model for gas source localisation is proposed. This algorithm is rule-based, and the confidence in a limited number of hypotheses is updated according to wind measurements. Rodriguez et al. [64] presented a wind field estimation algorithm for flying robots. The authors first estimated the wind vectors at different positions by combining different sensors, such as GPS, and then used the estimated wind vectors to fit Weibull distributions to model the wind vectors at different altitudes.

Another important work in the field of AFM presenting some similarities to the contribution presented in Chapter 4 is the work by Bennetts et al. [7]. Works of Bennetts et al. [7] and work presented in Chapter 4 aim at addressing a problem of building a spatial model of turbulent air flow in a joint orientation-speed 
space. However, the major difference is the fact that I provide a partially continuous representation while Bennetts et al. [7] build a discrete representation.

In the end, it is important to mention, that building map of flow using vector fields, which up to some extent can reassemble velocity fields, is investigated in the field of computer vision (i.e. Ali et al. [1]).

\subsubsection{Trajectory Mapping}

Trajectory mapping approaches, in brief, aim at finding a reliable method to extract from data paths followed by objects and clustering them in a meaningful way, each of the clusters is represented as a single trajectory. These methods have received great attention in the field of computer vision [52], where they have been used, among others, for surveillance and anomaly detection. Even though that a substantial body of work on trajectory mapping comes from the computer vision community, it is also a topic of interest in the domain of robotics and intelligent vehicles.

The problem of object tracking has received a substantial amount of attention over the years $[5,25]$. However, the results of tracking can still suffer from errors, such as noisy track estimates and broken or incomplete trajectories (tracklets). These errors can directly affect the quality of resulting trajectory maps.

The initial step in trajectory mapping is to obtain information about objects tracks. In the majority of the approaches, the obtained track information is further processed according to an activity-based semantic scene model, as described in work of Makris and Ellis [46]. This model contains two types of elements: points of interest (POIs) such as Entry/Exit points or Stop areas, and activity paths (APs) connecting POIs. The procedure of building such a model consists of following three tasks: obtaining tracks of objects, discovering POIs, building APs.

In the field of robotics, trajectory mapping is also used in numerous cases for improving human tracking. In 2005, Bennewitz et al. [9] presented an approach where information about usual human tracks is used to derive a Hidden Markov Model (HMM), which is further used for prediction of human motion. Moreover, they also show how to use probabilistic belief about the potential trajectories of persons in the path planning process.

Lefèvre et al. [41], points out that information about the trajectory patterns within the environment can substantially improve trajectory prediction capabilities, and in this way improve the planning and also increase the safety of autonomous vehicles.

Trajectory mapping is a well-developed family of approaches, which has received substantial attention over the years, mostly in the computer vision community. In spite of this, it still suffers from problems related to noisy and incomplete tracks. In cases, when the tracks are incomplete, it might be difficult to associate tracks to appropriate clusters. Moreover, incomplete tracks 
can create false POIs and APs; it is especially prominent in cluttered environments with multiple occlusions. In such difficult environments, tracking systems cannot usually provide a complete track of an object, and only provide a set of tracklets. That may lead to the situation where large amounts of data are discarded in the preprocessing step or might require a substantial effort to recombine them in to complete trajectories.

The methods presented in my thesis aims at building local models of dynamics and can use almost all available data even if they do not cover the complete trajectory. The methods presented in my thesis does not rely on the idea of POIs and APs instead, they attempt to build a model of dynamics around given locations in the map. Therefore, they do not require complete trajectories, what allows to utilise the tracklets. 



\section{Chapter 3 Modelling Motion Patterns with Conditional Transition Map}

\subsection{Introduction}

The work presented in this chapter is built on the assumption that the sensors used provide streams of data of discrete points in time. Each of the frames or scans provide a snapshot of the environment which is different from the previous one because of the changes caused by dynamic objects in the observed part of the environment. In this work I use data coming from a static sensor, however, presented approach can also work with moving sensor. The fundamental method to detect motion from such a stream of snapshots is to compare consecutive frames or scans to identify spatial configuration changes. The observed differences are evidence of dynamic changes in the environment.

Most robots perceive the external world through sensors providing data at discrete moments in time, e.g., cameras offer frames, or laser range sensors provide scans. In order to provide data, a sensor needs to collect measurements during a period, which is in the case of sensors considered in this chapter rather short. The laser scanner Sick LMS500 Lite, for example, provides scans with a frequency from $25 \mathrm{~Hz}$ to $75 \mathrm{~Hz}$ (depending on the configuration). That means that a new scan is published by the sensor every $0.04 \mathrm{~s}$ to $0.013 \mathrm{~s}$, respectively. These durations describing the time between publication of consecutive scans also defines the upper bound for the measurement time. In other words, the measurement time cannot be longer the time between publication of two scans. For many practical applications, these periods of time are short enough, to assume the resulting stream of data consists of a sequence of static snapshots of the environment.

One way to detect spatial configuration changes (dynamics/changes in the map caused by moving objects) is to build a sequence of occupancy grid maps, where each grid map is build using one of the consecutive quasi-instantaneous measurements. Once the grid maps are constructed, the goal is to build a model 


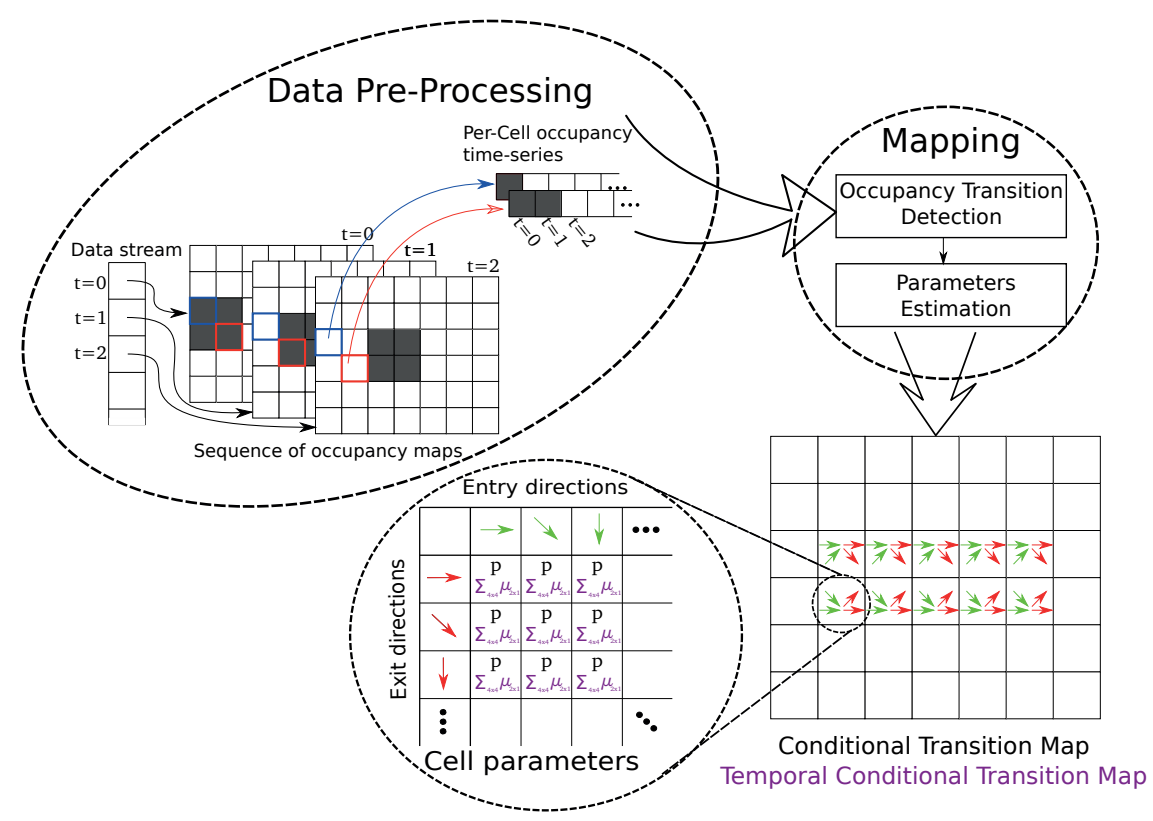

Figure 3.1: An overview of the Conditional-Transition Map (CT-map) and its extension Temporal Conditional-Transition Map (T-CT-map) (In the figure elements unique for T-CT-map are marked magenta).

In the data pre-processing step a discrete stream of data (e.g. a sequence of laser scans) is converted into a sequence of binary occupancy grid maps. The sequence of occupancy grid maps is used to provide a time series describing occupancy changes for each cell in the map. In the mapping step, dependencies between time series are detected and used to establish how the occupancy shifts between the adjacent cells. Based on these detected occupancy shifts parameters of CT-map or T-CT-map are computed.

The parameters of the model are sets of probabilities connecting entry of occupancy (green arrow) with an exit of the occupancy (red arrow). These probabilities describe the probability of a given entry-exit pair to occur $(p)$ - for both CT-map and T-CT-map. On top of that T-CT-map also stores the information about the relation between the duration of the entry and exit as a normal distribution (defined by its mean $\mu$ and covariance $\Sigma$ ).

of occupancy changes in each cell. However, the changes of occupancy are very often caused by objects moving along continuous trajectories through the environment. Therefore, models treating each cell independently, such as the ones reviewed in Sec. 2.2.1, lose a substantial amount of information about the dynamics in the environment. 
In this chapter I present Conditional-Transition Map (CT-map) and its temporal extension Temporal Conditional-Transition Map (T-CT-map). CT-map and T-CT-map are 2D representations which relax the assumption about cell independence that are common in alternative representations (see Sec . 2.2.1). CT-map and T-CT-map model the directions of spatial configuration changes by learning the dependency between the direction from which the occupancy arrived and the direction to which the occupancy is heading.

Fig. 3.1 shows in brief how to build CT-map and T-CT-map and also presents the parameters of the representations. In the initial step, a sequence of snapshots of the environment is converted into a sequence of binary occupancy grid maps. Then, from the sequence of occupancy grid maps time series describing occupancy changes per cell are extracted. Afterwards, the time series are evaluated in order to provide information how the occupancy is shifting between adjacent cells. Finally, the detected occupancy shifts are used to estimate the parameters of CT-map and T-CT-map.

The CT-map associates to each cell a set of conditional probabilities. These probabilities describe the probability of occupancy shift in a direction conditioned on the occupancy arriving from the given direction. T-CT-map also adds a distribution modelling the duration of the occupancy shift.

\subsection{Dynamics Extraction for T-CT-map and CT-map}

\subsubsection{Assumptions}

As mentioned in Chapter 2 an underlying assumption for building MoDs is that the dynamics follow patterns. However, the methods assuming cell independence acknowledge only the existence of temporal patterns. In the real world we observe not only temporal but also spatial patterns (e.g. cars following traffic rules). Therefore, the methods discussed in this chapter rely on the following implication: if motion of dynamic objects is following spatial patterns, then the occupancy changes caused by a motion of such objects will also follow spatial patterns. In order to build a map representing dependencies among adjacent cells, the stream of input data has to satisfy the following requirements.

2D Information The CT-map and T-CT-map are build based on sequences of 2D occupancy grid maps.

Resolution Limits CT-map and T-CT-map models the spatial dependencies only among the adjacent cells. Therefore, the spatial resolution should be such that the fastest moving object is never going to move further than one cell at a time. Otherwise, it will be impossible to identify the correct the dependencies between occupancy changes of adjacent cells. Therefore, the spatial resolution of the input grid maps, and in consequence the resolution of CT-map and 
T-CT-map, should depend on the temporal resolution of the used sensor and the expected maximum velocity of dynamic objects to be modelled.

\subsubsection{Data Pre-Processing}

The process of building CT-map and T-CT-map starts with converting a sequence of sensor readings into a sequence of binary occupancy grid maps, where each map corresponds to a single sensor reading. Afterwards, from the sequence of binary occupancy grid maps time-dependent occupancy signals $\left(G_{X, Y}(T)\right.$ where $(X, Y)$ denotes the spatial index of a cell) for each cell in the map are extracted:

$$
G_{X, Y}(T)=\left\{\begin{array}{cc}
1 & \text { if a part of an object is in the cell } \\
0 & \text { if free } \\
-1 & \text { if unobserved }
\end{array} .\right.
$$

After this step, each cell in the map has an associated binary value describing its state in discrete moments in time. According to Fig. 3.1 the next two steps in building CT-map and T-CT-map are Occupancy Transition Detection and Parameters Estimation.

\subsubsection{Occupancy Transition Detection}

In Fig. 3.2 we can see a sequence of time-stamped maps of a toy example. In the toy example a one-dimensional object, of size equal to two cells, is passing through a $1 \mathrm{D}$ environment from left to right. Each map in the sequence corresponds to a snapshot of the $1 \mathrm{D}$ environment while the object is moving.

Assume that we have observed this environment for an extended period and have seen the object passing from left to right and collected the corresponding series of maps multiple times. Relying on the collected observations, we can expect that if occupancy enters a cell from left, it will leave it to the right. This same statement can also be expressed as dependency among adjacent cells. Let us consider cell 4 (shown in Fig. 3.2 with a solid red border) a central cell of a 2-neighbourhood build out of cells 3 and 5. In such defined 2-neighbourhood in combination with the central cell the previous statement can be expressed as if occupancy shifts from cell 3 to 4 then it is very likely (according to past observations) that the occupancy will further shift from 4 to 5.

Let us now consider a more complex two-dimensional environment. Fig. 3.3 shows a model motion pattern at a roundabout. In the figure two transitions are marked, which can be described in the following way: if occupancy shifts from a lower middle cell it is very likely it will further shift to the left middle cell or the upper left cell.

As we can see based on these two examples occupancy changes in one cell are affected by occupancy changes in its neighbours. Intuitively, the occupancy 


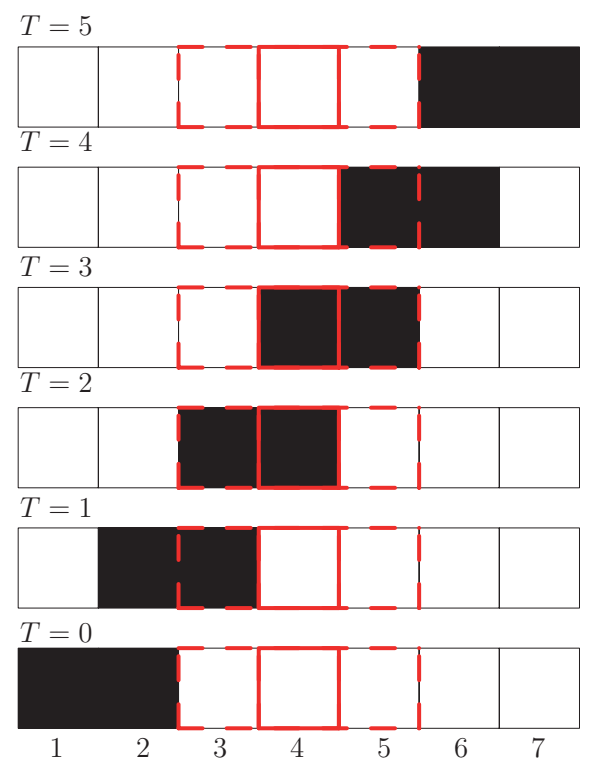

Figure 3.2: A sequence of maps representing spatial changes in a pure $1 \mathrm{D}$ environment caused by an object moving from left to right. The object occupies two cells at each time step. The solid red line marks edges of a central cell, and the red dashed line marks adjacent cells.

shifts from one cell to another. However, this is not the only dependency occurring in the examples; there is also the dependency between the shifts themselves. In Fig. 3.2 there is a dependency between the shift of occupancy from cell 4 to 5 and 3 to 4 . Similarly, in Fig. 3.3 there is shown a conditional dependency between the occupancy shifts marked with the green arrow and the yellow arrows.

Until now I have been discussing the problem of cell dependency relying on an intuitive understanding of such terms as a central cell, neighbour, transition and shift. Below, I will define these terms as they will be used in the context of CT-map and T-CT-map.

Neighbourhood (8-Neighbourhood) 8 -Neighbourhood is composed out of 8 cells (neighbours) surrounding one common central cell (all of the neighbours are directly adjacent to the central cell). In the remainder of this chapter, I will use only 8-neighbourhoods, thus for brevity, I will use term neighbourhood instead. In Fig. 3.2 and Fig. 3.3 the neighbourhood is marked with a red dashed line, and the central cell has a solid red edge. 


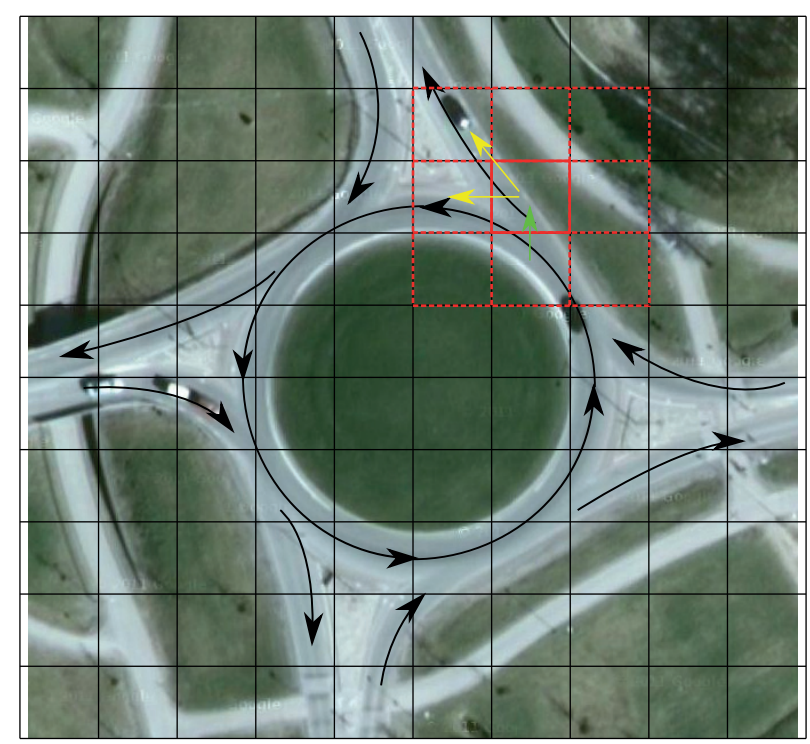

Figure 3.3: Roundabout in front of Örebro University. The black arrows represent the actual direction of motion. The red lines mark a neighbourhood. The green arrow represents an example entry direction to a central cell. The yellow arrows represent the associated directions.

Shift Let us consider two adjacent cells sharing a side or a corner. A shift occurs when one of the cells changes state from 0 to 1 (see Eq. (3.1)) and afterwards, the second cell becomes occupied as well. Furthermore, we can say the shift was from the first to the second cell. The shift occurs only if there is an interval when both cells are occupied or if the first cell changes state from 1 to 0 , and simultaneously the second cell changes state from 0 to 1 .

Entry Shift When the shift is from a neighbour to the central cell.

Exit Shift When the shift is from the central cell to a neighbour.

Transition A transition is two temporally and spatially dependent entry and exit shift. The entry and exit shift are spatially and temporally dependent when the occupancy shift of a central cell being a part of entry shift is simultaneously a part of an exit shift.

Onset and Offset In my work, I assume that the occupancy signal $\left(G_{i}(T)\right)$ of an $i$-th cell is binary. Therefore the onset $\left(s_{i}(T)=1\right)$ at time step $T$ occurs when 
the cell was free in the previous time step and became occupied, while offset $\left(s_{\mathfrak{i}}(T)=-1\right)$ occurs when the cell changes state from occupied to free:

$$
s_{i}(T)= \begin{cases}1 & \text { if }\left(G_{i}(T-1)=0\right) \wedge\left(G_{i}(T)=1\right) \\ -1 & \text { if }\left(G_{i}(T-1)=1\right) \wedge\left(G_{i}(T)=0\right) \\ 0 & \text { otherwise }\end{cases}
$$

\section{Onset Detection for Transition Detection}

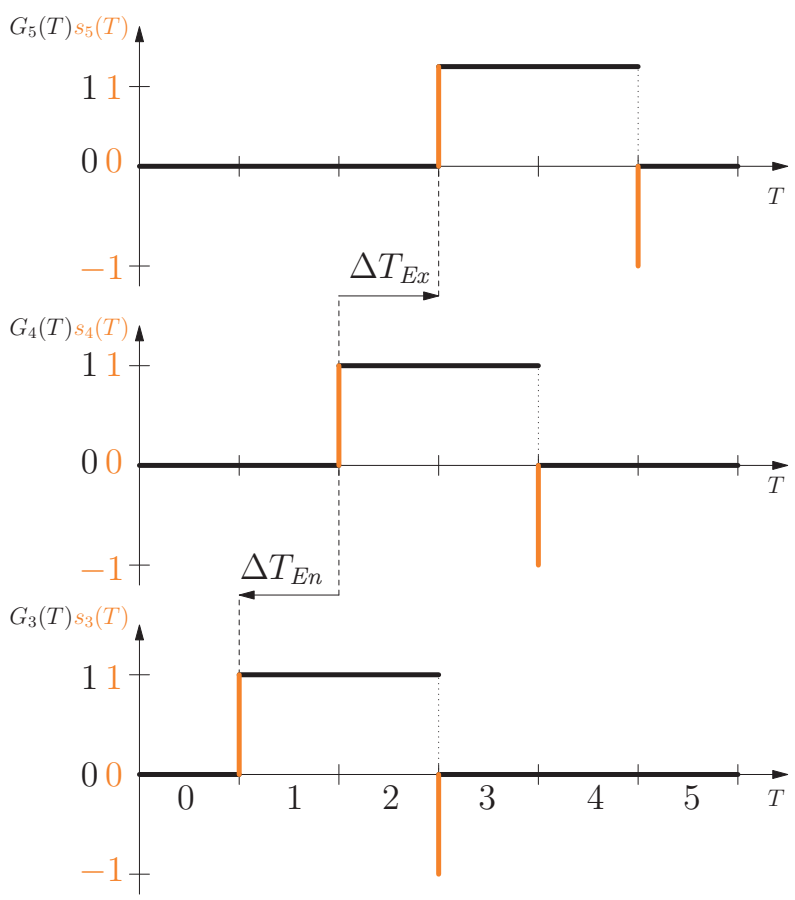

Figure 3.4: Example of transition detection with onset detection approach. The three plots present the occupancy signal $\left(G_{i}\right)$ in time for the cells in the neighbourhood marked in Fig. 3.2. The orange line marks the position of the detected onsets $\left(s_{i}\right)$ according to Eq. (3.2). Finally, $\Delta T_{E n}$ is a duration of an entry shift, namely a time between the onset of an adjacent cell $\left(\mathrm{G}_{3}\right)$ and the central cell $\left(\mathrm{G}_{4}\right)$, while $\Delta \mathrm{T}_{E x}$, is a duration of an exit shift, namely the time between the onset of the central cell $\left(\mathrm{G}_{4}\right)$ and an adjacent cell $\left(\mathrm{G}_{5}\right)$.

The implementation of transition detection can be derived directly from its definition. In Fig. 3.4 we can see three plots representing changes of occupancy over time for three cells forming the red-marked neighbourhood in Fig. 3.2. 
The first step is to detect the onset for each signal as defined in Eq. (3.2). Fig. 3.4 shows detected onsets as orange lines.

The second step is to find the onset for a neighbouring cell which occurred earlier than the onset in the central cell. Afterwards, it is necessary to check if the neighbour was occupied when the central cell became occupied. If yes, then it is an entry shift. In order to detect the exit shift, it is necessary to check if the central cell was occupied when there was an onset in a neighbour, which experienced onset last.

The transition detection with onset detection is a fast and lightweight method. However, as it considers every onset as an element of a new transition, it makes this implementation vulnerable to noisy occupancy signals.

\section{Cross-Correlation Based Transition Detection}

Real-world occupancy signals will often suffer from noise, which adds false onsets in the occupancy signal. These faulty onsets will cause that the implementation presented in the previous section will signal false transitions. To address the issue of faulty onsets, at least partially, I suggest using cross-correlation for transition detection.

Cross-correlation is a measure of similarity of two series as a function of displacement. In other words, cross-correlation allows us to find the lag (temporal offset) for which the dot product of two time-series is the highest. However, this type of analysis, cannot be performed with complete occupancy signals but it should consider only intervals, which should be corresponding with occupancy caused by the same moving object. The robustness of cross-correlation based transition detection lies not in the cross-correlation itself but in the method to extract such intervals. In contrast to onset based transition detection, crosscorrelation based transition detection considers intervals, which may contain false onsets caused by noise, instead of checking each onset separately.

Cross-Correlation Intervals Extraction The extraction of cross-correlation interval is a process to find a starting moment in time, before which two cells in the neighbourhood and the central cell were free, and an ending moment in time, after which the two cells in the neighbourhood and the central cell are free again. The process is repeated for all the pairs of cells in the neighbourhood (two neighbours and the central cell).

In order to understand the procedure of building such intervals let us analyse the example of two objects moving through a one-dimensional environment: two objects moving with the same speed in opposite directions. One from left to right and another one from right to left. In Fig. 3.5 we can see the corresponding time series describing the occupancy changes in cells $3,4,5$ using the one-dimensional map shown in Fig. 3.2.

The dashed green and red lines correspond to onsets and offsets of each occupancy interval. In order to find the beginning of a cross-correlation interval, 
we check before which onset all the cells were free for at least one time-step. Similarly, we look for the end of a cross-correlation interval by checking after which offset all of the cells become free for at least one time-step. The beginning and end of cross-correlation intervals are marked with solid green and red line.

As mentioned above the extraction of the cross-correlation interval is the key for robustification of the process of transition detection. However, in highly congested environments it can also lead to merging occupancy corresponding to multiple objects following very closely each other into one cross-correlation interval. In my work I do not address this problem, mostly because did not affect the map building process; however, it should be addressed in order to increase the robustness of the algorithm.

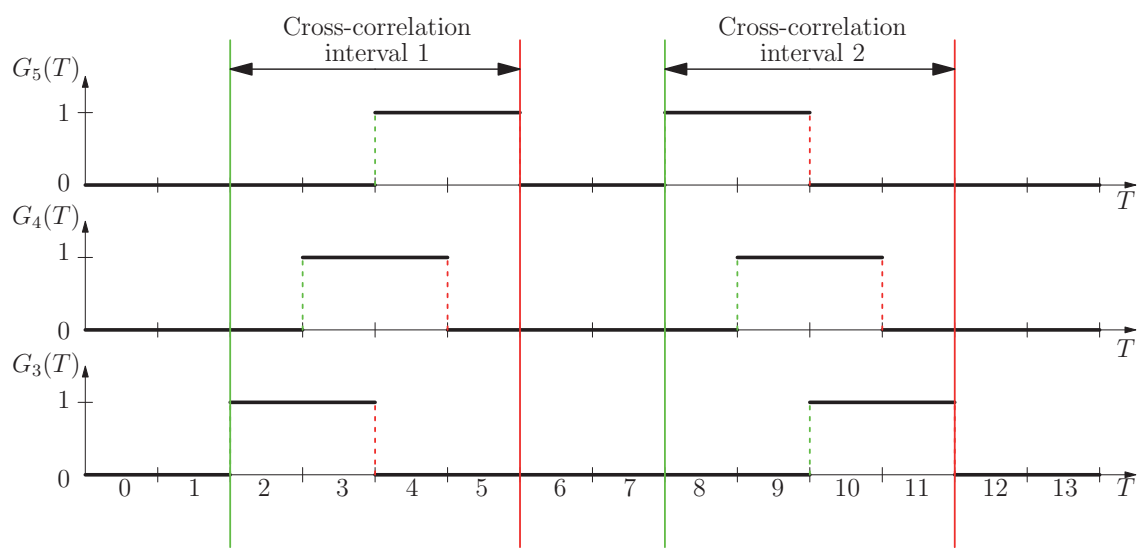

Figure 3.5: Example of cross-correlation interval separation. The two solid green lines mark beginning of two cross-correlation intervals, and the solid red lines mark the respective ends of the cross-correlation intervals. The beginning of the first cross-correlation interval is equivalent to the moment when occupancy signal of cell number $3\left(G_{3}(T)\right)$ changes from free to occupied. Before this moment all the cells were free for at least one time step. The beginning of the second cross-correlation interval is marked for this same reason by the change of occupancy for cell number $5\left(\mathrm{G}_{5}(\mathrm{~T})\right)$. The end of the cross-correlation intervals is marked by the moment in time after which all three cells are free for at least one time step.

Cross-Correlation Cross-correlation is a process, where the evaluated signals are shifted with respect to each other by all possible lags at the given discretisation of time. However, in work presented in this chapter the occupancy signals 


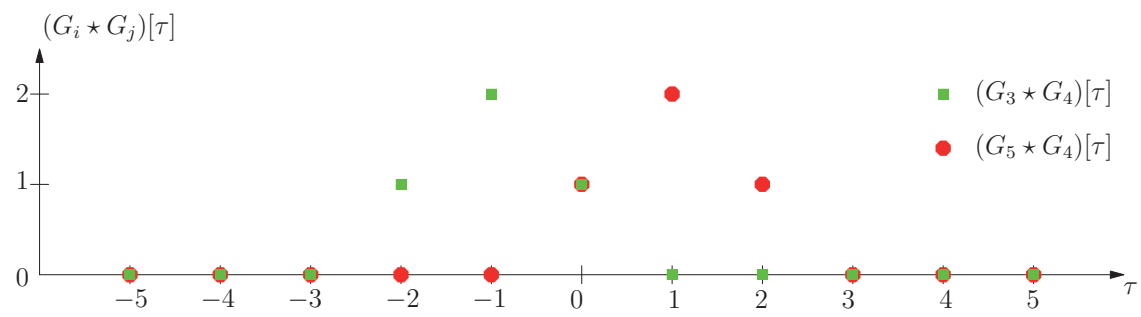

Figure 3.6: Plot of values of cross-correlation $\left(G_{i} \star G_{j}\right)$ for occupancy signals of cells 3 and 4, and 5 and 4 (as shown in Fig. 3.4) with respect to lag $(\tau)$. The green plot has its peak for $\tau_{\max }=-1$, which corresponds to entry shift, while the red plot has its peak for lag $\tau_{\max }=1$ which corresponds to an exit shift.

are finite, therefore the lag $\tau$ is bound to an interval defined by the length $\mathrm{N}$ of the signal:

$$
\tau \in[-\mathrm{N}, \mathrm{N}]
$$

For each lag $(\tau)$, an inner-product is computed for the two signals $\left(G_{i}\right.$ and $\left.G_{j}\right)$. Cross-correlation for two finite occupancy signals of equal length $N$ can be denoted as follow:

$$
\left(G_{i} \star G_{j}\right)[\tau]=\sum_{T=0}^{N-\tau-1} G_{i}[T] G_{j}[T+\tau] .
$$

Let us now consider occupancy signals (see Fig. 3.4) corresponding to cells 3, 4, and 5 for the environment shown in Fig. 3.2. To find the exit and entry shifts, we have to compute the cross-correlation of the occupancy signal of cells 3 and 4 and 5 and 4 . The plot of resulting values of cross-correlation is shown in Fig. 3.6. We can see peak-values for the cross-correlation corresponding to the lag resulting with the best overlap. The lag of cross-correlation corresponding to the peak for cells 3 and 4 is negative, indicating an entry shift, while the exit shift occurring between cells 5 and 4 is resulting in a positive lag.

The detection of a shift with the cross-correlation can be described in the following way. First step is to find a lag $\left(\tau_{\max }\right)$ corresponding to a global maximum of value of cross-correlation between occupancy signals $G_{i}$ and $G_{j}$ of $i$-th and $j$-th adjacent cells.

$$
\Delta \mathrm{T}_{i, j}=\underset{\tau}{\arg \max }\left(\left(\mathrm{G}_{\mathrm{i}} \star \mathrm{G}_{\mathrm{j}}\right)[\tau]\right)
$$

The second step is to check if the lag $\left(\Delta \mathrm{T}_{i, j}\right)$ corresponds to entry shift $(E n)$ or exit shift $(E x)$ :

$$
S h_{i, j}= \begin{cases}E n & , \text { if } \Delta T_{i, j}<0 \\ E x & , \text { if } \Delta T_{i, j}>0\end{cases}
$$




\section{Temporal Aspect of Occupancy Shift}

In the majority of the cases, the occupancy shift will take more than a single time-step. This time is inversely proportional to the velocity of the object. The T-CT-map incorporates the information about the duration of an entry and an exit shift. Both onset based transition detection and cross-correlation based transition detection provides this information. In case of the onset transition detection, it is the time between the onset of the neighbouring cell and the central cell. In case of the cross-correlation transition detection, it is the lag corresponding to the peak value of cross-correlation between the neighbour and the central cell.

\subsubsection{Transitions}

As mentioned above the transition is a spatially and temporally connected entry and an exit shift. Considering the one-dimensional examples presented in Sec. 3.2.3, the importance of the concept of transition might not be clear at first glance. Therefore, let us consider a toy example in a two-dimensional case.

Fig. 3.7a shows an intersection where an equal number of objects follow one of two paths, either from left upwards or from right downwards. If the representation only provides the information about the exit shifts, then the resulting map, at least in some parts, would be ambiguous (i.e. the middle of the intersection). Fig $3.7 \mathrm{~b}$ shows an example of such an ambiguity.

The ambiguity can cause a problem in the following example. Let us assume that to the cell with solid orange border occupancy has shifted from the right. Our goal is to predict in which direction the occupancy will shift further. If the used representation is only considering exit shifts, then it is impossible to predict in which of four possible directions the occupancy will move.

To address the problem of the ambiguity, I suggest incorporating in the map not only the information about the exit but also the information about the entry shifts. Fig. 3.7c shows all the possible transitions (entry and corresponding exit shifts) for the central cell (solid orange border).

Let us now consider the solution for our problem of predicting the further shift of the occupancy. Thanks to the information that occupancy arrived from right it is now reasonable to assume that it is more likely that the occupancy will shift either directly downwards or diagonally leftwards. This prediction is not free from ambiguity; however, this time at least the result is more coherent.

We can conclude the toy example saying that, if transitions (sequences of entry and exit shifts) are considered it is possible to model entry-dependent exit directions - otherwise the model would have to marginalise overall entry directions and would be less expressive.

Finally, it is important to emphasise that one entry shift can have multiple exit shifts associated with it, as shown in Fig. 3.7c. This can be caused by one of two facts. First, the dynamics might be following such a pattern in this 


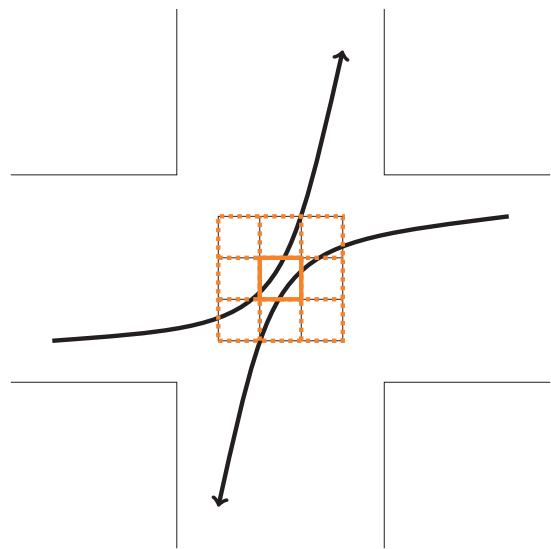

(a) General patterns followed by objects in the intersection with an overlaid neighbourhood.

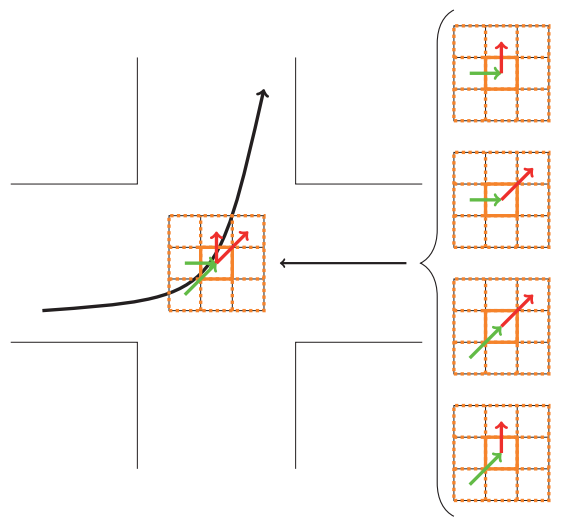

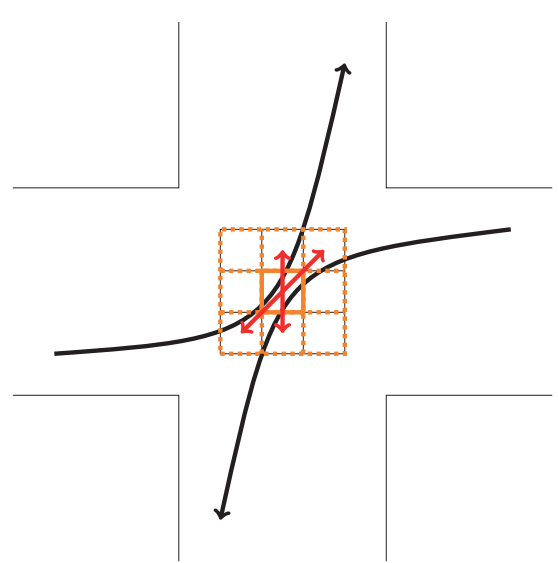

(b) The red arrows represent the exit shifts for the central cell. The exit shifts are pairwise opposite what results in an ambiguous information regarding the possible transitions.

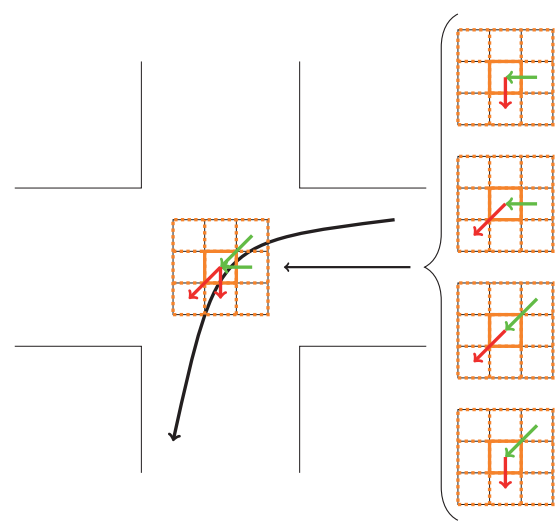

(c) A set of eight transitions. The green arrows represent entry shifts, and the red arrows represent the exit shifts. In order to increase the transparency of the representation, the transitions were split. On the left, there are transitions associated with the leftupwards pattern, and on the right, they are associated with the right-downward pattern. Moreover, on the right of each map each transition is presented on separated sub-map.

Figure 3.7: An example of motion pattern for a simple intersection. The additional information regarding the entry directions, even though that increase the size of the map, allows to encode the motion patterns present in the environment properly. 
location (i.e. T-shaped intersection). Second, objects usually occupy multiple cells at once, therefore also the shift of occupancy occurs into multiple cells at once.

\subsection{The Representations of Dynamics}

\subsubsection{Conditional Models}

At the end of the previous section, I have presented a toy example showing the benefits of a map containing information not only about the exit shifts but also about the entry shifts. One of the ways to model the dependency between the entry and exit shift is to use conditional probabilities. Conditional probability is a measure of the probability of an event given that another event has occurred. In the case of the previously presented example, the conditional probability expresses the probability of the exit downwards if the occupancy arrived from the right.

The conditional models described in this section focus on building model of the local behaviour of occupancy changes. Such models have two advantages compared to models assuming cell indepence (Sec. 3.2.3). First, conditional models contain information about the direction and speed of occupancy shifts across cells borders and do not just model state changes per cell. Second, conditional models have more predictive capabilities in contrast to models which focus on storing the past observations.

\section{The Conditional Transition Map}

The CT-map is a grid-based representation, which models the probability of transition as a conditional probability of an exit shift $(E x)$ given an entry shift (En). Fig. 3.8 shows a set of exit shifts (red arrows) with associated conditional probabilities conditioned on occupancy having entered the central cell from cell $\mathrm{D}$ (e.g. the probability of the occupancy exiting from central cell to cell $\mathrm{E}$ if the occupancy arrived from cell $\mathrm{D}$ is equal to $\left.\mathrm{P}\left(E x_{\mathrm{E}} \mid E n_{\mathrm{D}}\right)\right)$.

The complete CT-map associates to each cell in the map a set of eight conditional probabilities for each one of eight possible entry directions. This means that the representation has to maintain 64 parameters per cell.

\section{The Temporal Conditional Transition Map}

The major shortcoming of CT-map is that it ignores the temporal aspect of transitions. To address this shortcoming, I propose to include in the model the information about the duration of entry and exit shift. The duration of the exit and the entry shift provides not only information about the speed but also about the acceleration or deceleration. The speed can be computed as the ratio between the spatial resolution of the grid map and the duration of exit 


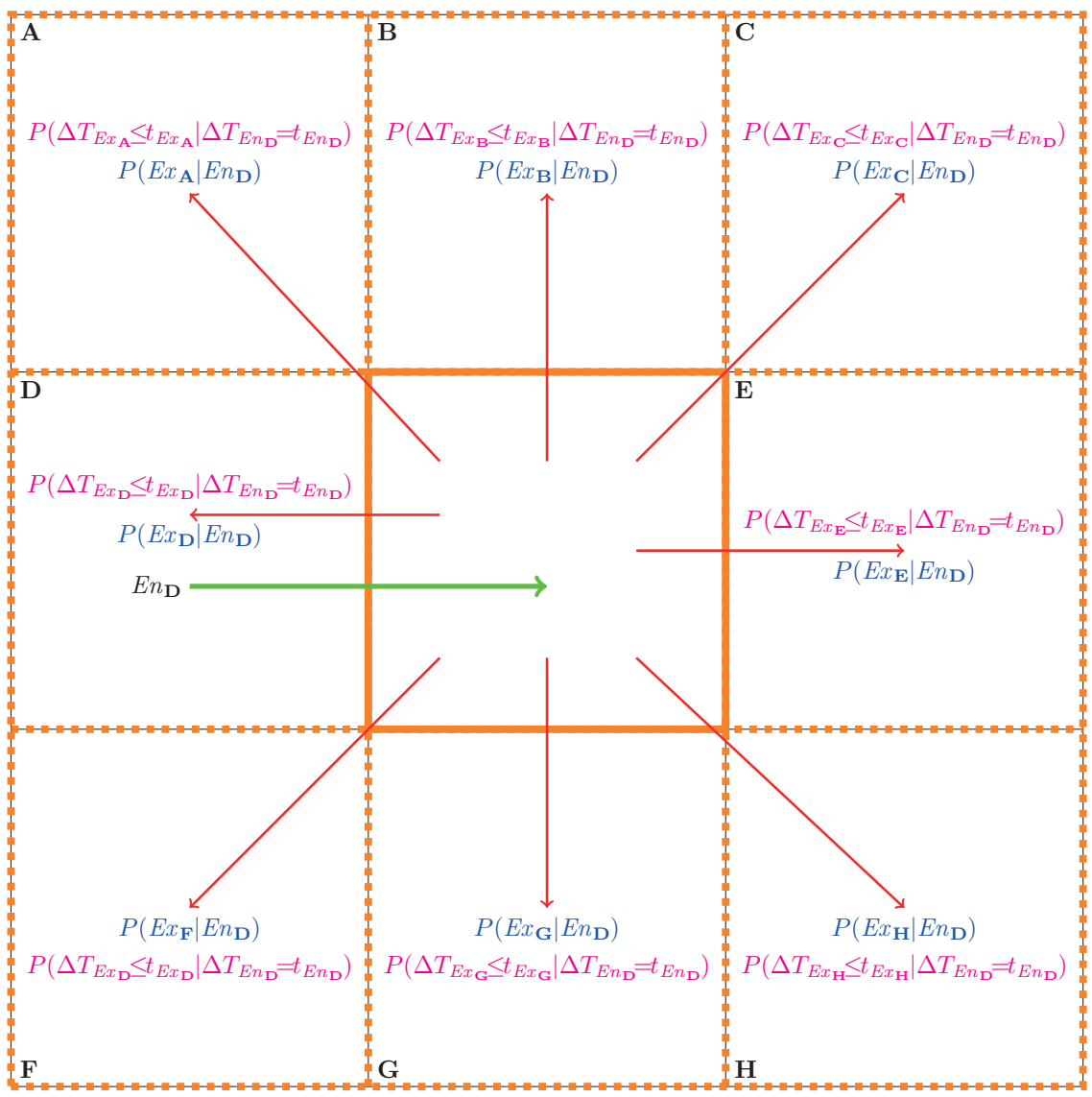

Figure 3.8: A set of conditional probabilities of a T-CT-map binding an entry shift (green arrow) from cell D $\left(E n_{\mathrm{D}}\right)$ with exit shifts $E x$ (red arrows). The T-CT-map is an extension of CT-map, both representations share the conditional probability of transition (expressions marked blue); however, T-CT-map also carries information about the dependency between the entry and exit shift duration (expressions marked magenta). 
and entry shift, while the acceleration can be estimated based on the difference between the speed of the entry and exit shift.

To address the temporal aspect of transitions, I propose to extend the set of parameters describing a cell in a CT-map with a conditional probability describing the dependency between the duration of exit shifts based on the duration of an entry shift. This extended version of CT-map is called T-CT-map. Fig. 3.8 shows a set of exit shifts with associated conditional probabilities considering the entry direction and the entry shift duration.

The entry duration $\left(\Delta T_{E n_{\mathrm{D}}}\right)$ is the interval between the change from free to occupied in the cell $\mathrm{D}$ and the central cell. It can be interpreted as the time necessary for the front of the object to travel from the border of the cell $\mathrm{D}$ to the border of the central cell. The exit duration to cell $\mathrm{E}\left(\Delta \mathrm{T}_{E n_{\mathrm{E}}}\right)$ is defined similarly and corresponds to the interval between the change from free to occupied in the central cell and the cell E.

The distribution of the transition time is modelled as a bivariate normal distribution representing the joint distribution of the entry and the exit durations, where $\mu$ denotes the distribution's mean and $\Sigma$ denotes distribution's covariance matrix. Furthermore, $\mu_{\mathrm{T}_{E n}}$ and $\sigma_{\mathrm{T}_{E n}}$ denotes the mean and variance respectively of the entry duration, while $\mu_{\mathrm{T}_{E x}}$ and $\sigma_{\mathrm{T}_{E x}}$ denotes the mean and variance of the exit duration. Finally, $\rho$ is the correlation between exit end entry duration.

$$
\begin{gathered}
\boldsymbol{\mu}=\left[\begin{array}{c}
\mu_{\mathrm{T}_{E n}} \\
\mu_{\mathrm{T}_{E x}}
\end{array}\right] \\
\boldsymbol{\Sigma}=\left[\begin{array}{cc}
\sigma_{\mathrm{T}_{E n}}^{2} & \rho \sigma_{\mathrm{T}_{E n}} \sigma_{\mathrm{T}_{E x}} \\
\rho \sigma_{\mathrm{T}_{E n}} \sigma_{\mathrm{T}_{E x}} & \sigma_{\mathrm{T}_{E x}}^{2}
\end{array}\right]
\end{gathered}
$$

\subsubsection{Parameter Learning}

In the previous section I have emphasised that T-CT-map is an extension of CT-map and both representations share some parameters; therefore, in this section, I will discuss the learning procedure for CT-map and T-CT-map jointly and flag only the differences between these two representations.

\section{Off-Line Learning}

The offline procedure of building the CT-map and T-CT-map is as follows:

1. Creation of a sequence of occupancy grid-maps.

2. Extraction of binary occupancy time series for each cell.

3. Detection of occupancy transitions in the neighbourhood. (see Sec. 3.2.3)

4. Computation of set of conditional probabilities for each transition for each central cell. The conditional probability $\mathrm{P}\left(E x_{\mathrm{I}} \mid E n_{\mathrm{J}}\right)$ is computed as 
ratio between exit shifts to cell $\mathrm{I}$ if the entry was from cell $\mathrm{J}$ to all the entry shifts from cell J.:

$$
\mathrm{P}\left(E x_{\mathrm{I}} \mid E n_{\mathrm{J}}\right)=\frac{\# E x_{\mathrm{I} \mid \mathrm{J}}}{\# E n_{\mathrm{J}}}
$$

5. (Valid only for T-CT-map) Computation of the normal distribution parameters to model the duration of exit and entry shifts. The input data in this step are pairs of durations $(\mathbf{t})$ describing a corresponding entry $t_{E n_{J}}$ and exit shift $\mathrm{t}_{E x_{\mathrm{I}}}$ :

$$
\mathbf{t}=\left[\begin{array}{l}
\mathrm{t}_{E n_{\mathrm{J}}} \\
\mathrm{t}_{E x_{\mathrm{I}}}
\end{array}\right]
$$

In order to compute the mean value $\mu$ and covariance matrix $\Sigma$ of the joint distribution of the exit and entry duration based on $\mathrm{K}$ observations a maximum likelihood estimator is used:

$$
\begin{gathered}
\boldsymbol{\mu}=\frac{1}{\mathrm{~K}} \sum_{\mathrm{k}=1}^{\mathrm{K}} \mathbf{t}_{\mathrm{k}} \\
\boldsymbol{\Sigma}=\frac{1}{\mathrm{~K}} \sum_{\mathrm{k}=1}^{\mathrm{K}}\left(\mathbf{t}_{\mathrm{k}}-\boldsymbol{\mu}\right)\left(\mathbf{t}_{\mathrm{k}}-\boldsymbol{\mu}\right)^{\mathrm{T}}
\end{gathered}
$$

\section{On-Line Learning}

The off-line procedure for parameters learning works well with both transition detection methods, the onset detection and the cross-correlation based one. However, the primary drawback is that it does not account for the possibility to update the existing model. Furthermore, it requires to store the whole history of observations until the parameter estimation takes place, it might bring a substantial burden especially for large data-sets.

Similarly to off-line learning procedure also online learning procedure has to address an issue of updating two sets of parameters per cell: the conditional transition probability and the distribution of entry and exit durations.

In case of a probability of transition, the online process of parameters estimation can be expressed as an update of counters corresponding to each transition and computation of the probability when needed (see Eq. (3.9)).

To compute the normal distribution describing transition times, it would not be feasible to store all observations. Therefore, I will use a method for recursive update of mean and covariance, introduced by Chan et al. [17] for onedimensional data and extended to a multivariate case by Saarinen et al. [67]. However, in contrast to work of Saarinen et al. [67] in recursive computation of T-CT-map parameters, at each step only one sample is added. 
In this procedure it is necessary to collect $m$ initial samples and estimate their mean $\boldsymbol{\mu}_{m}$ and matrix $\boldsymbol{S}_{m}$, which is defined as $\boldsymbol{S}_{m}=\sum_{i=1}^{m}\left(\mathbf{t}_{i}-\boldsymbol{\mu}_{m+1}\right)\left(\mathbf{t}_{i}-\right.$ $\left.\mu_{m+1}\right)^{\top}$. Once, initial values of $\mu_{m}$ and $S_{m}$ are computed the updated procedure consists of two elements.

First, a new mean value is computed $\mu_{m+1}$.

$$
\mu_{m+1}=\frac{1}{m+1}\left(m \mu_{m}+t\right)
$$

Second, a new value of $\mathbf{S}_{\mathrm{m}+1}$ is computed, which later on is used to compute an update covariance matrix $\Sigma_{m+1}$

$$
\begin{aligned}
& S_{m+1}=S_{m}+m\left(\mu_{m}-\mu_{m+1}\right)\left(\mu_{m}-\mu_{m+1}\right)^{\top} \\
& +\left(\mathbf{t}-\boldsymbol{\mu}_{\mathrm{m}+1}\right)\left(\mathbf{t}-\boldsymbol{\mu}_{\mathrm{m}+1}\right)^{\top} \\
& \Sigma_{\mathrm{m}+1}=\frac{1}{\mathrm{~m}} \mathbf{S}_{\mathrm{m}+1}
\end{aligned}
$$

\subsubsection{Conditional Probability Propagation Tree}

The Conditional Probability Propagation Tree (CPP-tree) is a tool to extract global motion patterns stored in a CT-map. It is a tree representing all executable transitions (with probability greater than 0 ) connected to the initial entry shift.

Fig. 3.9 shows the procedure of building a CPP-tree: each leaf of the tree is treated as a possible entry shift. Once all possible entry shifts are detected, a CT-map or T-CT-map is queried in order to find all corresponding exit shifts. Once all exit shifts are detected, they are added to CPP-tree.

Information provided by CPP-tree can be used either to estimate the future paths of tracked objects or to extract reachable areas for path planning. Depending on the desired outcome different picking strategies can be pursued. One of the picking strategies is to choose all possible transitions. This builds a map of all reachable cells. Another picking strategy is to always pick the most likely transition, in this way a single branch presenting a most likely path will be obtained.

To lower memory consumption I do not store every possible path from the root to leaves separately. Instead of this, I store the information about the set of immediate successors of every exit, that is the following exit.

It is also possible to compute the likelihood for each path in the CPP-tree. The likelihood of a path is computed as a joint probability of all the transitions constituting the path.

$$
\mathrm{P}(\text { path })=\prod_{i=1}^{\mathrm{N}} \mathrm{P}_{\mathrm{i}}(E x \mid E n)
$$




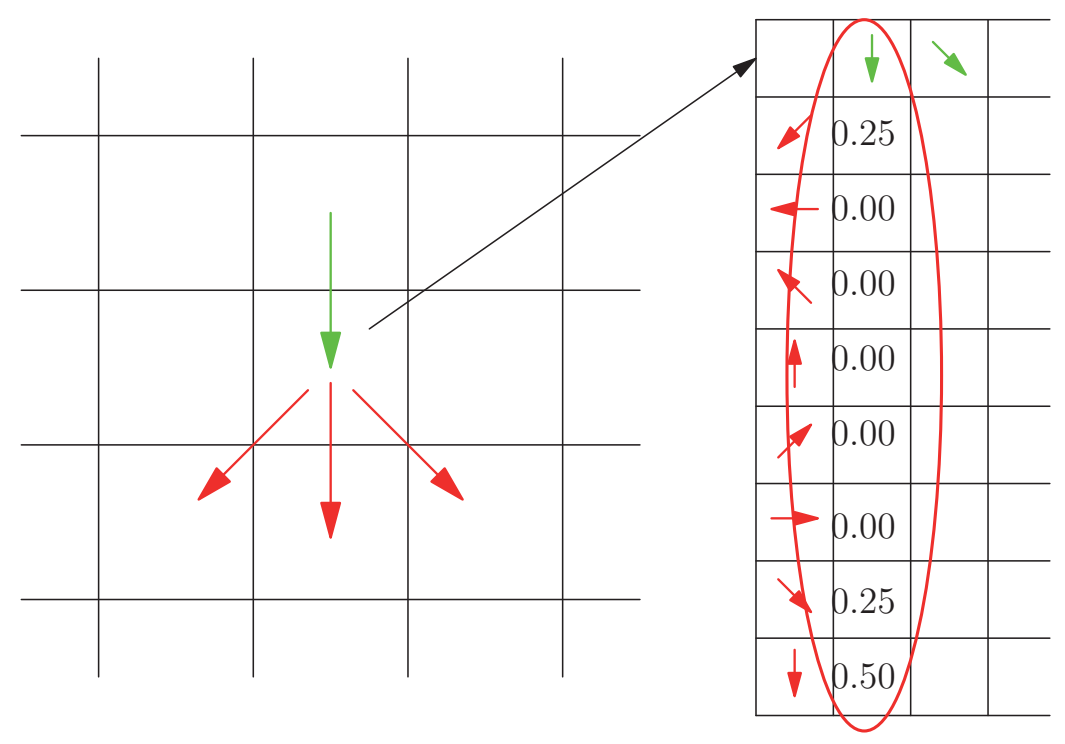

Figure 3.9: In CT-map and T-CT-map an exit shift of one cell is an entry shift of its neighbour. Following this idea, the way to build a CPP-tree is to find all exit shifts in the tree that do not have any following shifts. Then we assume that this exit shift is an entry shift to a next cell. Based on this entry shift (green arrow) we query the conditional probabilities to find all possible exit shifts (red arrows). Then these exit shifts are added to CPP-tree, and in next step, they will be treated as entry shifts for a new layer.

where $\mathrm{N}$ is the number of considered transitions in the path.

\subsection{Mapping Results}

The focus of this section is to present results of building CT-map and T-CT-map for synthetic and real-world data.

\subsubsection{Toy Example}

Fig. 3.10 shows a toy example. In the figure, we can see a test environment, where a purple rectangle marks the starting position for an object travelling through the environment left to right. The environment is overlaid with a grid map. Fig. 3.10 shows a grid map corresponding to a configuration, where an object is in a start position (the occupied cells are marked with a wavy pattern). In the run of the simulation, a dataset containing observations of an object 


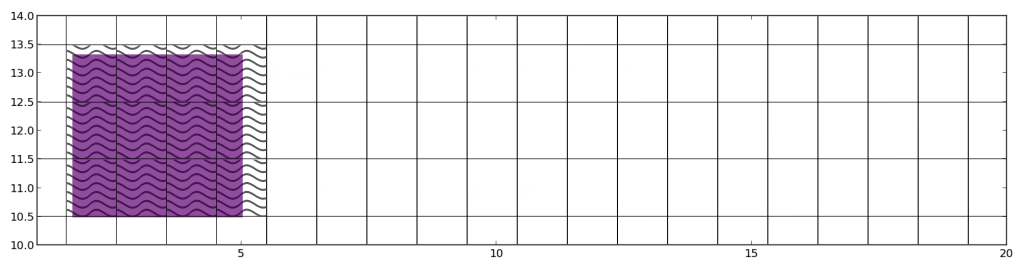

Figure 3.10: A setup for a toy example. The purple square marks a starting position of object travelling from left to right through the environment. In this toy example, object will travel for times with four different constant speeds. Furthermore, the environment is overlaid with a grid map. In the figure, we can see a grid map corresponding to a configuration where an object is in the start position. The wavy pattern marks occupied cells.

passing through the environment four times with different constant velocities was collected. Based on the collected data a T-CT-map was constructed.

Fig. 3.11a shows exit shifts for transitions where the entry shift is from left. For the clarity of the visualisation, I am not showing the exit shifts associated with the entry shifts form the other directions. In Fig. 3.11a, we can see that for each entry shift there are associated two or three exit shifts. The reason for such a behaviour is that the objects in the toy example occupy multiple cells at once. Therefore, when the object moves, it enters multiple cells along its front edge simultaneously. From a neighbourhood perspective, this looks like the occupancy has moved from a central cell to multiple neighbouring cells.

The four objects were moving with four different, constant velocities. These differences in speed are affecting the temporal part of the discussed model (T-CT-map). The entry and the associated exit time measurements are marked in Fig. 3.11b as black dots. The elongated shape of the distribution and the $45^{\circ}$ tilt are caused by the fact that the objects were travelling with constant but different speeds. This means that exit and entry time per object were similar, almost equal, but they were different for each object. If the velocities of the objects were changing in time, then the tilt and the shape of the distribution would also be affected, reflecting the observed accelerations.

In Fig. 3.12 we can see two Gaussians corresponding to two different motion patterns. First shows the distribution representing the duration of exit and entry shift of objects moving with constant, but different, speeds. Second shows the distribution representing the duration of exit and entry shift of objects slowing down with different ratios.

The more vertical and broader Gaussian represents a case where objects are moving relatively fast in the first place and slows down afterwards. The shape of the Gaussian is inferred from the entry and exit times. For this distribution we can see that the entry times are relatively short, approximately one second, 


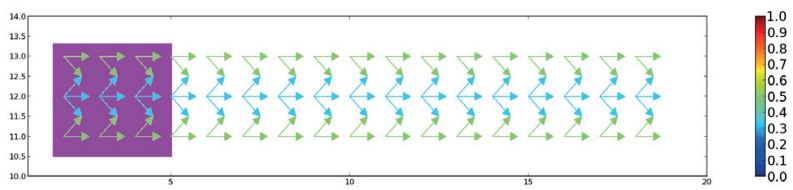

(a) Visualisation of the T-CT-map for data collected with the example presented in Fig. 3.10. The arrows represent exit directions, and their colour coding corresponds to conditional probabilities of exit shifts if entry shift was from left, the other shifts were omitted for clarity. (The explanation for such behaviour of the map can be found on page 43).

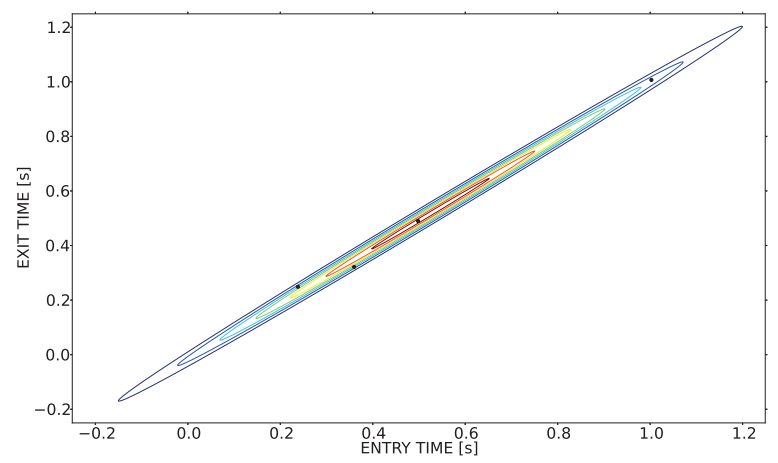

(b) A bivariate distribution of durations of exit and entry shifts for a single transition in the map. The black dots mark the measured combinations of entry and exit times.

Figure 3.11: The resulting T-CT-map for the toy example presented in Fig. 3.10.

while the exit times are longer; indicating lower speed (deceleration). The thin and diagonal Gaussian represents a case where objects are moving with different velocities; however, they tend to maintain the velocity.

In this toy example, we can see that the proposed mapping method can model the direction of occupancy shift on a cell level. This is a substantial advancement with respect to methods assuming cell independence, which would only mark a part of the environment as dynamic. However, the resulting map may be perceived as ambiguous because the occupancy shift is not always parallel to a direction of motion of the object (there are diagonal exit shifts). This ambiguity is limiting if the application is to perform object tracking; however, it should not be an issue if aiming at predicting the future occupancy.

Fig. 3.11b shows an estimated distribution of duration of entry and exit shifts. The primary advantage of such a representation is that it represents information about velocity and acceleration or deceleration of moving objects at 


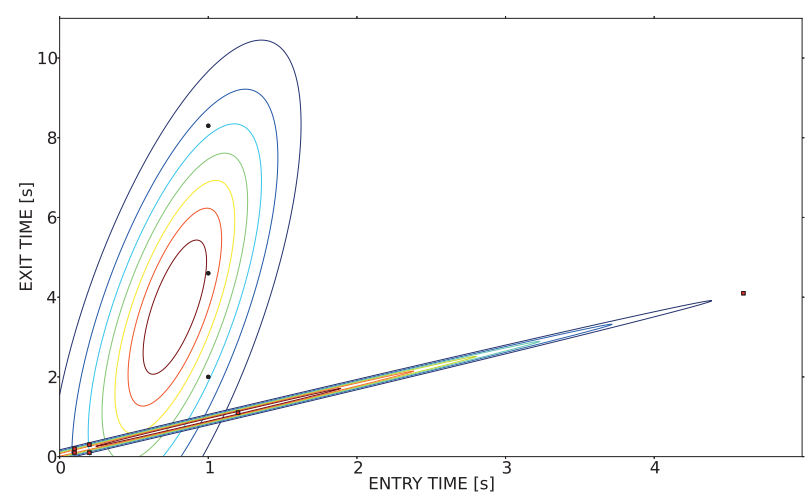

Figure 3.12: Gaussians representing bivariate distributions of two different motion patterns. The black dots are the data points used to compute the distribution parameters. The Gaussian distribution on the left represents a case where objects are slowing down; therefore the exit time tends to be much longer than the entry time. The thin Gaussian is describing a case where objects tend to maintain their speed in between entering and exiting the considered cell.

a particular location. If the velocity is constant, then the mean value of the velocity distribution will be situated along the diagonal of the plot. Acceleration and deceleration will cause the main axis to be shifted towards the Y-axis and $\mathrm{X}$-axis respectively.

However, the proposed exit and entry shift duration model has two significant limitations. First, the model assumes unimodality of the collected data. In some cases this assumption is justified (e.g. for cars on a highway that tend to maintain a constant speed). However, in multiple real-world scenarios, the environment will contain areas of heterogeneous dynamics (e.g. mixed bike paths and pedestrian's ways). In such areas, a uni-modal distribution is not an appropriate way to model the joint distribution of entry- and exit durations.

The second issue of the described representation is the assumption that the data is following a normal distribution. The normal distribution is symmetric and its mode and mean are overlapping. However, in real-world cases, it might happen that the measurements are following a skewed distribution, in such a case the mean and mode do not overlap anymore. In such a case the estimation of a dominant entry and exit shift durations as a mean value is not valid. Furthermore, the predictions obtained with normal distribution are faulty i.e. it might predict transitions with negative durations.

It is especially prominent for asymmetric distributions where the highest probability of durations will be shifted away from the true one. Furthermore, if the durations are short, it might lead non-zero probabilities for negative durations. 
A third issue is that the increased expressiveness leads to increased memory consumption. Saarinen et al. [66] requires only five parameters to be stored per cell, what is substantially fewer in comparison to 64 parameters needed by CT-map and 384 parameters required by T-CT-map. However, for both CT-map and T-CT-map the amount of used memory depends only on the size of the map expressed as a number of cells $(n)$ and will remain constant independently from the number of observations. Therefore, the memory complexity for the worst case scenario is expressed as $\mathrm{O}(n)$. However, in the average case (map also contains some static areas) the memory consumption can be reduced using dynamical allocation of memory and maintaining the variables only for the locations where the changes of occupancy were observed.

\subsubsection{Mapping with CT-map}

\section{Data Set Description}

To demonstrate the capabilities of CT-map I have used a data set collected with a Velodyne-HDL64 3D laser scanner situated at the centre of the roundabout shown in the Fig. 3.3. The considered environment consists of a typical urban environment with typically dynamic regions (roads, sidewalks) typically static regions (lawns), static obstacles (trees, fences) and dynamic obstacles (cars, humans). Velodyne-HDL64 provides measurements at $10 \mathrm{~Hz}$ and the 3D data is projected to $2 \mathrm{D}$ by using the ROS node velodyne_obstacles from the package velodyne_height_map ${ }^{1}$.

\section{Qualitative Analysis of an Example of CT-map}

Fig. 3.3 shows a top view of the test environment. The considered environment contains very few obstacles that can effectively limit the motion of cars and humans. However, there is a set of rules that affect the behaviour of a dynamic object within the environment. Such rules, if known during the design of the robotic system, can be included in the robots code. In the case when these rules are not known in the implementation phase, they can be learned during the runtime. One of the methods to learn these rules is to build a MoD. The MoD can extract the spatial structure of the dynamics in the environment.

In Sec. 2.2.1 I discuss two types of methods mapping dynamics in grid map. The maps of type 1 can provide information about the shape of the dynamic areas and also provide information about the intensity of the motion. However, they fail to provide information about the direction of objects motions. In the case of the roundabout (see Fig. 3.3) map providing information only about the intensity of motion (e.g. Saarinen et al. [66]) motion will provide only information about the shape of the roundabout and the roads and pavements around it. In contrast CT-map provides information that (at least in some parts) the

\footnotetext{
${ }^{1}$ http://www.ros.org/wiki/velodyne_height_map, used version 0.3.0.
} 


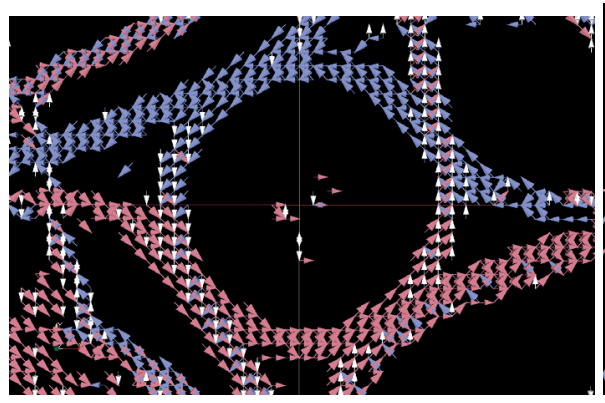

(a) Pattern of motion in the roundabout environment shown in Fig. 3.3.

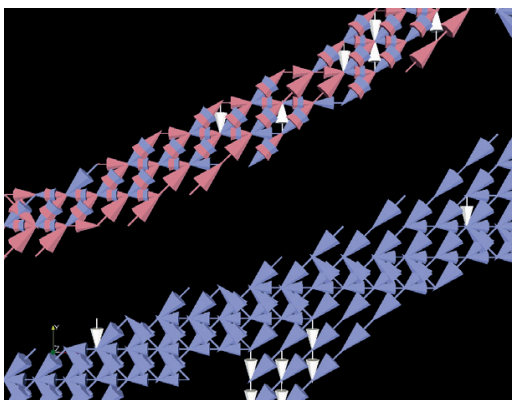

(b) A pattern of motion for sidewalk (upper one) and road (lower one). On the sidewalk, we can see two streams with opposite directions. On the road, we can see a split in a stream.

Figure 3.13: A visualisation of CT-map revealing motion patterns on a roundabout. For better visualisation, the edges with less than 10 exit shifts were removed, and entry directions are not shown. (The cell size is $2 \times 2 \mathrm{~m}$. The colours refer to the orientations of vectors.) In the centre of Fig. 3.13a visible transitions are corresponding to the motion of the experimenters mounting the sensor. Further, it is visible the structure of the roundabout consisting of the central ring and the approach roads. Finally, in the top left corner, one can see a sidewalk where people go in two opposite directions. In the bottom left corner, we can see noisy transitions caused by moving trees. Fig. $3.13 \mathrm{~b}$ shows a closeup on a pavement and the road. The CT-map is preserving not only a coherent flow of cars, going in this same direction but also the motion of pedestrians which does not follow such rigid motion patterns.

dynamic objects are following a right-hand traffic (see Fig. 3.13a). This information has a substantial impact on robots behaviour and also can improve the quality of motion predictions.

Fig. 3.13b shows one more advantage of CT-map, the multimodality. CT-map is a representation, which can assign to each of the considered locations in the map. That allows the map to maintain the information motion patterns that are overlapping (e.g. the motion patterns on the sidewalk) or intersect.

The process of building a MoD relies on accumulating observations over time, in contrast to the process of building a regular spatial map. This requirement can substantially extend the time of map preparation and in consequence, delay the deployment. However, in many cases, it is possible to combine the map building process with robot's operation. To fully benefit from such combination it is necessary that map building algorithm can work with streaming data. CT-map provides such capabilities for both methods of learning re- 


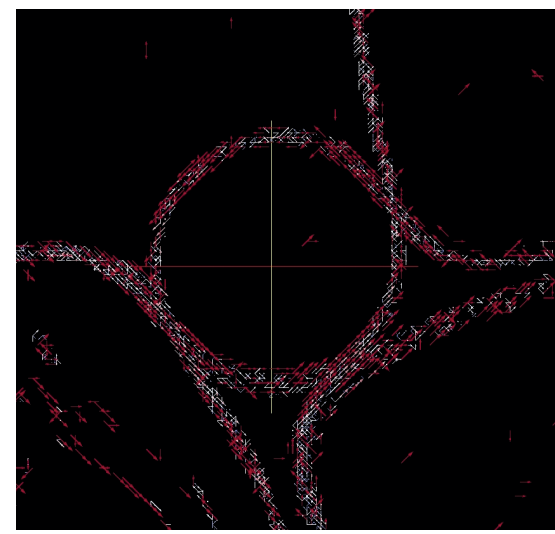

(a) CT-map after 0.5 minute of observation.

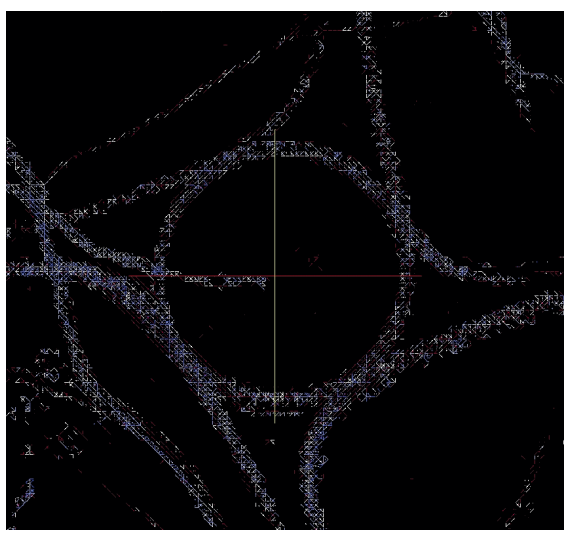

(b) CT-map after 2.5 minutes of observation.

Figure 3.14: The progress in a mapping process. The colours are corresponding to the conditional transitions. Very likely transitions appear in red, while less likely transitions appear in white-blue. By comparing Fig. 3.14a with Fig. 3.14b we can see that even from a small amount of data it is possible to build a meaningful MoD, which later on can be extended. This example shows the importance of online learning. In the presented example already after 30 seconds, the robot has some knowledge about motion patterns in the environment.

lies only on a short buffer which contains the most recent occupancy changes. Fig. 3.14 shows that already after a short observation time as thirty seconds it is possible to build an informative map of motion patterns in an urban scenario. However, the proposed learning methods do not include the way to forget past motion patterns. Therefore, whenever a change of motion pattern occurs, it is necessary to restart the mapping process.

\section{Qualitative Analysis of an Example of CPP-tree}

In Sec. 3.3.3 I have introduce so called CPP-tree. CPP-tree is a tool allowing to extract global motion patterns from CT-map, which is a local representation. CPP-tree extract global motion patterns by building a set of reachable transitions between adjacent cells, starting from a given initial transition. Fig. 3.15 shows an example of CPP-tree which depth is 500 . The resulting tree contains transitions around the roundabout and also on the exit roads, but, as desired, does not include the entry roads as unreachable regions. 


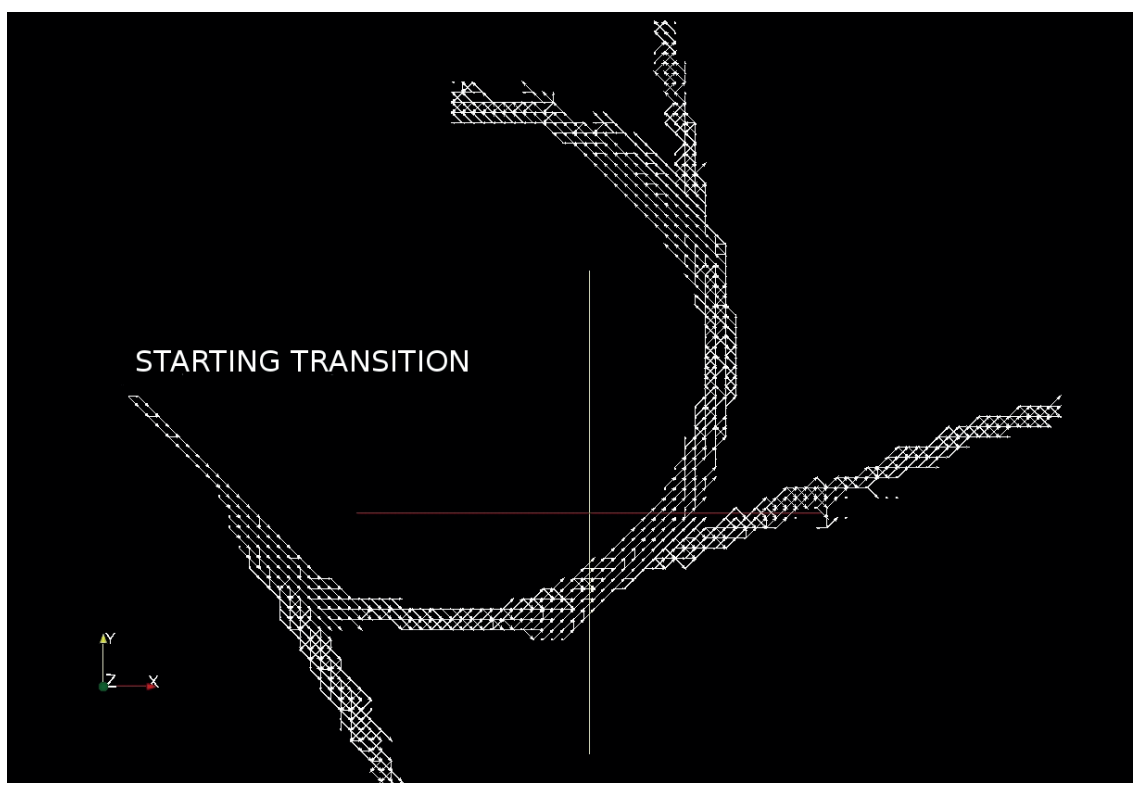

Figure 3.15: CPP-tree for a roundabout (cell size $0.8 \times 0.8 \mathrm{~m}$ ). It is a CPP-tree of depth 500 showing all reachable cells from the starting cell. We can see that the resulting areas are corresponding to the parts of the roundabout. Furthermore, the CPP-tree extended only on the exit roads but not on the pavement or entry roads. That is cause by the fact that there were no transitions from road to pavement.

\section{Summary}

In this section, I presented and discussed a CT-map built for a roundabout. The core idea behind CT-map is to model the dependencies between the occupancy changes of adjacent cells.

The qualitative study presented in this section highlights the benefits of using CT-map. In the first place, the CT-map not only contains information about dynamic areas but also, retains the information about the direction of motion. Furthermore, because CT-map is a multimodal representation it can retain the information about intersecting, overlapping and splitting flows. Additionally, the conditional probabilities help to separate intersecting or overlapping flows as shown with the extraction of a CPP-tree.

However, CT-map also has limitations. CT-map does not provide information about the time transitions take. Furthermore, the entry and exit shifts can only appear in one of eight directions, that might lead to ambiguity if there are motion patterns not aligned with these directions. Moreover, the model does 
not provide information about its uncertainty. Finally, to store the information about the occupancy flow each cell requires 64 parameters, which is more than some of the method assuming cell independence (i.e. independent Markov chain by Saarinen et al. [66] requires only five parameters per cell).

\subsubsection{Mapping with T-CT-map}

\section{Test data-set}

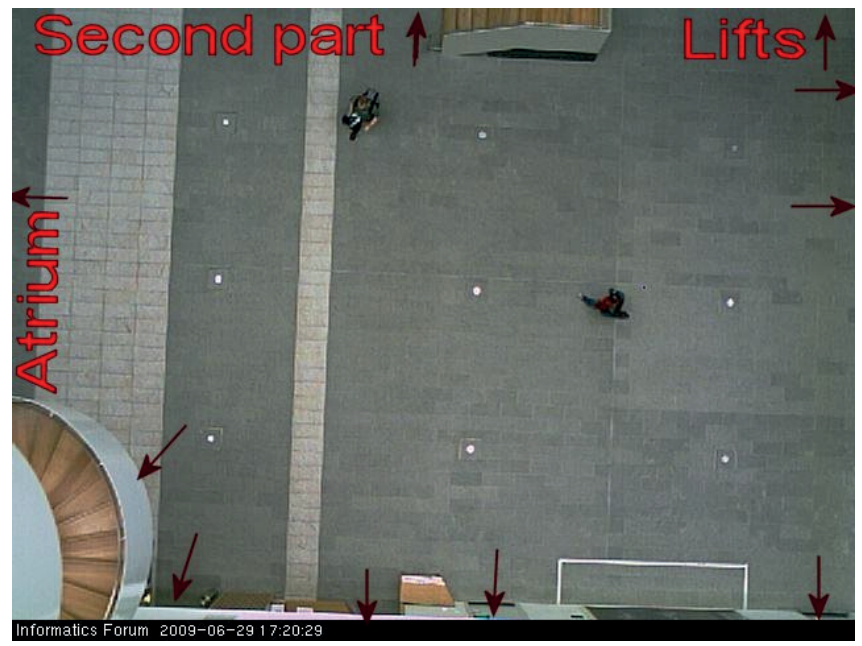

By courtesy of: Majecka et al. [45]

Figure 3.16: An image of a test environment - the Informatics Forum in the main building of the School of Informatics at the University of Edinburgh. The main entry/exit points (marked) are at the bottom left (front door), top left (cafe), top centre (stairs), top right (elevator and night exit), bottom right (labs).

In this section, I present a T-CT-map built from a data set collected by Majecka [45] using an overhead camera providing images with $640 \times 480$ resolution. The camera was fixed $23 \mathrm{~m}$ above the floor and covered an area of size 16 by 11 meters (see Fig. 3.16). The dataset provides information about the position and the size of the bounding box for each of tracked people for each frame.

In order to build T-CT-map, the input data need to be converted into a sequence of timestamped binary occupancy maps. To obtain such a sequence, I have used the information about the position of bounding boxes for each frame. Because the dataset contains all the parameters of the bounding boxes expressed as pixels, it is necessary to convert them to the floor coordinate frame. The conversation is based on the fact that each pixel (horizontally and verti- 


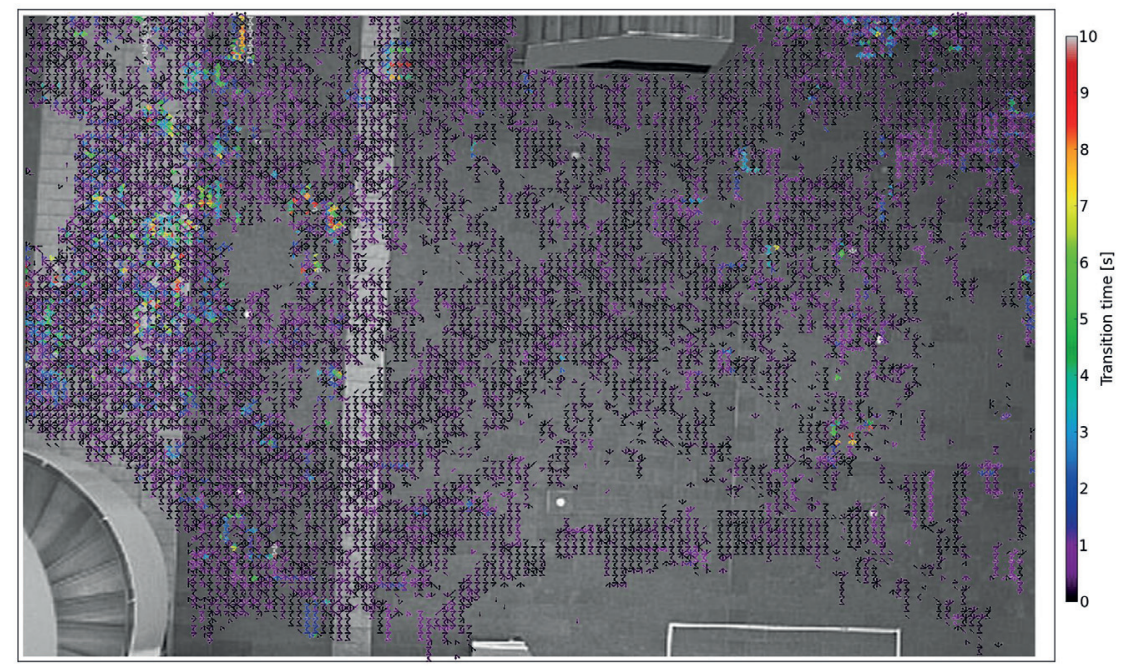

Figure 3.17: An image of the test environment with an overlaid T-CT-map. Arrows are indicating exit directions for entry from the right. The colour coding corresponds to the mean time measured to exit from a given cell to a neighbouring cell.

cally) corresponds to $24.7 \mathrm{~mm}$ on the ground. In the map building process, the bounding boxes are treated as if they are completely occupied. In the result, each frame is converted into a binary occupancy grid map with a cell of size $0.125 \mathrm{~m}$ by $0.125 \mathrm{~m}$. In this experiment, I have used a T-CT-map using the first $1.5 \mathrm{~h}$ of measurements from the 25th of August.

\section{Qualitative Analysis of an Example of T-CT-map}

Fig. 3.17 shows the image of the test environment overlayed with the exit shifts for entry shifts from the right; furthermore Fig. 3.18 shows four maps containing exit shifts for entry shifts from the top, bottom, left and right respectively. In Fig. 3.17 and Fig. 3.18 we can see that the motion patterns do not appear inside the obstacles (stairs on the top of the map) or in the occluded area (beneath a round staircase in the left bottom corner). Furthermore, it is also visible that some areas are free from dynamics even though they do not contain permanent obstacles (i.e. area in the bottom right of the map).

Fig. 3.18 also shows that depending on the direction of the entry shift the structure of the spatial motion patterns changes. The first visible difference is that if the entry direction is from top or bottom, the motion patterns in the right part of the map are diagonal. On the other hand for entry direction from the left or right the motion patterns in the right part of the map are horizon- 


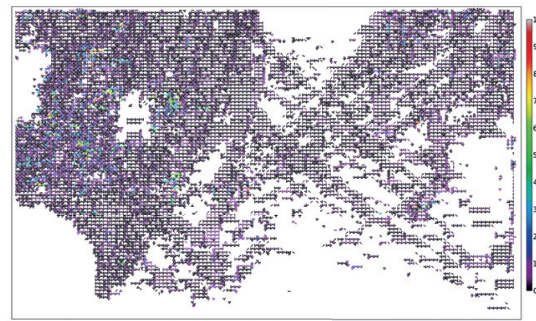

(a) Upwards

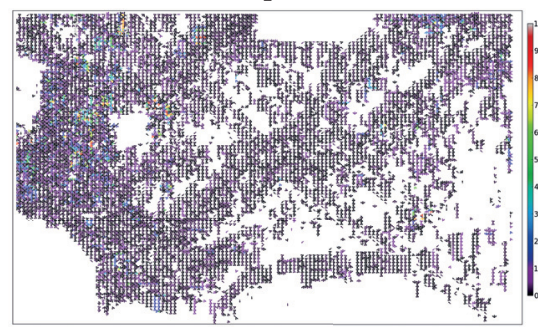

(c) Leftwards

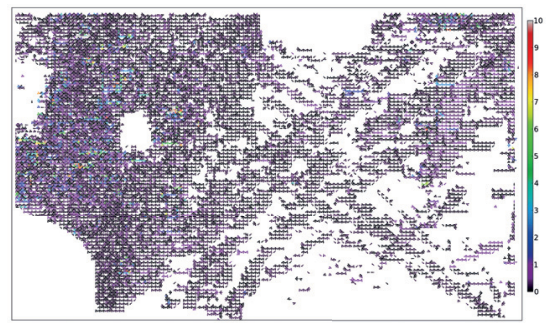

(b) Downwards

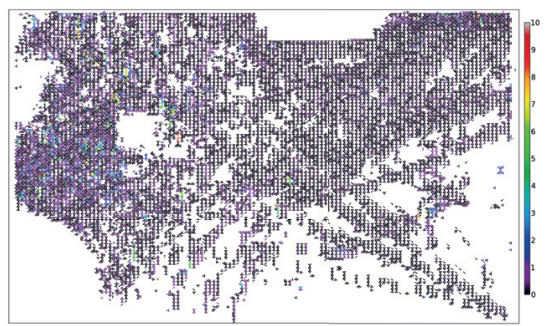

(d) Rightwards

Figure 3.18: Comparison of motion patterns depending on entry shifts. The colour coding corresponds to mean exit shift time as in Fig. 3.17. The figures show that depending on the entry direction different spatial motion patterns can be observed. For instance in Fig. 3.18c there is a visible pattern connecting left bottom corner with the middle of the bottom edge of the map, which is not visible on any other of the maps. A similar pattern is visible in the top part of the map where the spatial motion pattern is mostly from left to right or from right to left (Fig. 3.18c and Fig. 3.18d).

tal. Furthermore, in Fig. 3.18c there is visible a motion pattern connecting the centre of the bottom edge of the map with the right corner of the map. This motion pattern is not visible for any other entry direction.

Moreover, in Fig. 3.17 we can see how the time of exit from a cell depends on the location on the map. We can see that for the most of the area objects are moving relatively fast (they need approx. $0.1 \mathrm{~s}$ to cross a cell); however, we can observe areas where objects are more likely to slow down or even stop for some time. Such areas are visible near to some exits especially on the left side of the figure and in the top right corner, near to those areas are exits and lifts.

\section{Summary}

In this section, I have presented the capabilities of T-CT-map. T-CT-map extends the capabilities of CT-map to express the temporal aspect of transitions. 
The major limitation of this method is the fact that the transition probability and transition time are modelled independently. Furthermore, the representation is bounded to the grid map, sub-cell velocity changes cannot be modelled.

\subsection{Conclusions}

The work presented in this chapter concentrates on building representations able to model occupancy changes in a grid map caused by dynamic objects. However in contrast to methods of type 1 (see page 19), which assume that occupancy changes in adjacent cells are spatially independent, the representations discussed in this chapter model the dependencies between adjacent cells. Conditional-Transition Map and its temporal extension Temporal ConditionalTransition Map, are multimodal, parametric, conditional representations, which model the dynamics in the environment as flow of occupancy over a grid map.

\subsubsection{Contributions}

The first advantage of using CT-map and T-CT-map is an increased expressiveness of the model. In contrast to methods of type 1, which are only able to model the changes in cell occupancy, CT-map and T-CT-map are explicitly modelling the direction of occupancy shift. The direction information can be a substantial advantage in the planning process. A motion planner can build trajectories complying to implicit traffic patterns and minimise (or maximise) the interference with the dynamics in the area.

The second key advantage of using CT-map and T-CT-map is the conditional character of these representations. These two representations do not only provide information to where the occupancy can shift from a current cell, but they also consider the direction from which the occupancy has arrived. The importance of this information is especially visible in areas where the flow of occupancy overlaps (i.e.sidewalk).

Furthermore, T-CT-map also contains the temporal information. The information about the duration of entry and exit shifts implicitly denotes not only velocity but also velocity changes. T-CT-map to each of the shifts in the transition associates also the statistics describing their durations. If the durations are equal, that means the object tend to maintain their speed. However, any discrepancies between durations of entry and exit shift indicate also changes in the speed of the object. It is important to remember that these durations are computed based on the way the front of the object is moving across the map. Therefore, it is necessary to account for the size of the object while estimating when the cells will become free again.

Finally, the proposed methods are local, it means, that maps can be built out of incomplete data and easily updated without retraining the whole map. 


\subsubsection{Limitations and Future Work}

In the previous section, I have listed advantages of CT-map and T-CT-map. However, these methods also have some limitations and can undergo further development.

CT-map and T-CT-map are local methods, this, on the one hand, allows to build partial models from incomplete data, in contrast to global methods where incomplete data may make building model more difficult. However, it also causes that the global motion patterns (reaching beyond a neighbourhood) are not explicitly modelled. In order to extract them it is necessary use other techniques (i.e. CPP-tree).

Furthermore, CT-map and T-CT-map are parametric models limited by the underlying grid map. In the first place, they are not able to differentiate subpixel orientation differences neither sub-pixel velocity changes. T-CT-map also assumes, that the durations for entry and exit shift are unimodal and normally distributed. These assumptions can limit the quality of the predictions generated with this model.

CT-map and T-CT-map are limited to what type of dynamics they can represent. These representations are tailored to model motion patterns of discrete dynamic objects. That means, that motion of contains media or changes of the environment caused by semi-static objects can be modelled with neither of the representations.

In the first part of this section, I have presented limitations of the expressiveness of the representation. Another type of limitations is related to the procedure of parameter estimation of CT-map and T-CT-map.

CT-map and T-CT-map use as an input sequences of binary occupancy grid maps. The process of binarization can introduce additional noise, which can substantially affect both representations and the temporal part of T-CT-map especially. One of the ways to avoid this issue is to use the continuous occupancy grid map as input.

However, binarization is just one of the sources of noise. Therefore, a key research direction is robustification of the transition detection process. This task can be approached in at least to ways. The first approach is to detect the edges between occupied and free areas in the maps using an edge detector [11]. Once the edges in the maps are reliably detected this information can be used for detecting the shifts over the cell borders. The second approach focuses on processing the cells occupancy signals. This can include filtering the signal and application of robust step detection methods.

The further research is not only limited to improving the representations but also relates to possible applications. The two primary areas of application of CT-map and T-CT-map are motion prediction and motion planning.

In the case of motion prediction CT-map and T-CT-map can be used as an enhancement for Bayesian occupancy filter (BOF) [28]. BOF aims at predicting the future state of occupancy map based on the most recent changes in 
occupancy. In this case CT-map and T-CT-map can provide information about the motion patterns present in the environment. The information about motion patterns in the environment will help to improve the quality of the prediction and also enable BOF to make long time predictions.

The application of CT-map and T-CT-map is two fold. First, these representations can be used for estimation of a future shape of the environment in the presence of dynamic objects. Second, the information about the direction of flow of occupancy can help building flow compliant trajectories. Such trajectories are beneficial for two reasons. First, following already existing motion patterns in the environment may simplify and speed up motion planning process. Second, the information about motion patterns can help in building less obtrusive trajectories, which does not disrupt the usual motion patterns of objects in the environment. 



\section{Chapter 4 Modelling Motion Patterns with Circular-Linear Flow Field Maps}

\subsection{Introduction}

In Chapter 2 I presented and discussed different methods of perceiving and mapping dynamics (maps of spatial occupancy changes, velocity maps and trajectory maps); however, not all of these methods have received equal attention from the robotics community. In the robotics community, methods related to the mapping of spatial configuration changes attracted the greatest attention, while methods related to trajectory mapping were developed in the computer vision community. Velocity mapping was for a long time neglected and only recently received attention from the Mobile Robot Olfaction (MRO) community, where it is used to build airflow models. However, velocity mapping can be advantageous also for other tasks than Air Flow Mapping (AFM), for example, when building a MoD for human motion patterns.

In this chapter I introduce a representation called Circular Linear Flow Field map (CLiFF-map). CLiFF-map is a Gaussian mixture flow field, where to each location there is associated a multimodal distribution describing flow patterns around the given location. The idea of modeling a flow is shared by CT-map, T-CT-map and CLiFF-map. However, CLiFF-map is a substantial advancement, in that it is a continuous representation, over the work presented in the previous chapter.

Fig. 4.1 shows a bird's-eye view on the components of CLiFF-map and also the mapping process itself. In the figure, we can see that, CLiFF-map does not the model flow of occupancy changes in an underlying grid map but instead relies on velocity measurements. In the Data Collection frame, a robot is col- 


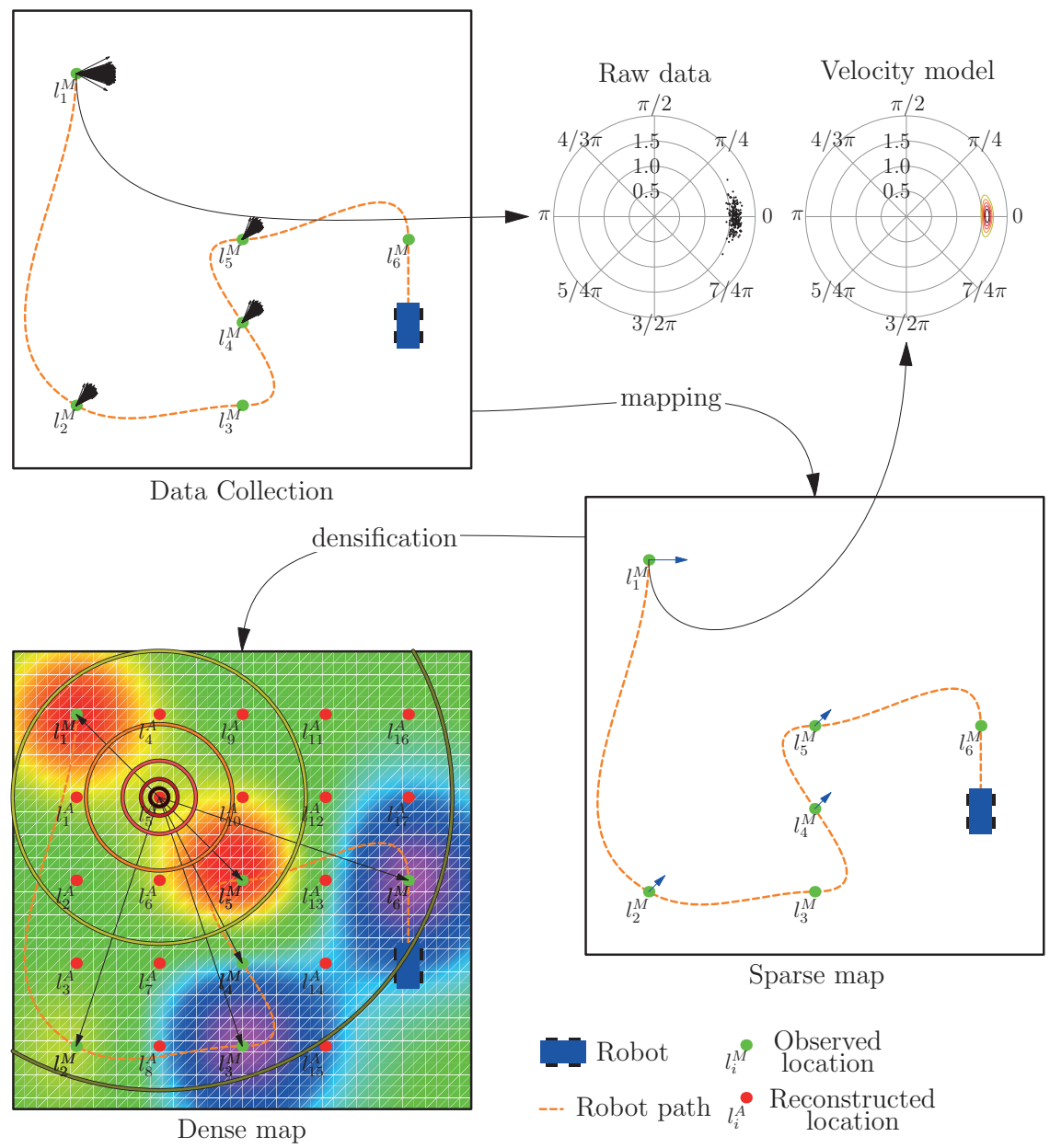

Figure 4.1: CLiFF-map mapping procedure. Data collection - A robot is travelling through an environment and collect velocity measurements. In four locations dynamics were observed (black arrows), in two locations there was no motion. Sparse map - Based on Raw data to each observed location in the map a probabilistic Velocity model is associated. Based on the amount of collected data a Gaussian Process in constructed, estimating the trust in the estimated velocity models in unobserved locations. Dense map - To obtain a dense map, information from observed locations are combined to estimate the velocity model in unobserved location. The influence of observed location is proportional to the distance and intensity of motion. 
lecting velocity measurements in the environment. These velocity measurements can be describing airflow or motion of people or cars.

During mapping, based on raw measurements, to each location there is associated a probabilistic velocity model. In the Sparse Map frame a resulting map is visible, each of the blue arrows represents the mean velocity of each cluster. CLiFF-map retains the information about a multimodal characteristic of the flow using Gaussian Mixture Models. The provided model of dynamics is not confined to a grid map with fixed discretisation and can express arbitrary orientations and speeds. CLiFF-map is also more expressive then CT-map and T-CT-map because it cannot only model the direction of motion but also explicitly include information about the relative intensity of motion and also provides a measure of trust in the model.

It is also important to emphasise that CLiFF-map allows building maps of motion patterns from incomplete or spatially sparse data (see Sec. 4.4). In the Dense Map frame, we can see how a dense map is built. From the distributions describing motion models in observed locations (green dots), a set of virtual observations is sampled. These virtual observations are then used to generate motion model for unobserved locations. Furthermore, the Gaussian Process is used to estimate the trust values for the unobserved locations.

One of the applications where it is beneficial is building maps of human motion patterns. The most common approach to model human motion patterns is to use trajectory maps. The process of building trajectory map starts with collecting tracks followed by people in the environment. However, the object tracking system is very often not able to provide complete trajectories between points of interest but tracklets (short trajectory fragments) instead. That substantially complicates the process of building a consistent trajectory map. In contrast, CLiFF-map does not require complete trajectory information and can build maps using only velocity estimates, provided either by the tracking system directly or estimated based on the timestamped trajectory points.

Finally, CLiFF-map allows also to build a dense map based on a sparse map. In the process of densification, the parameters of distribution in unobserved locations are estimated based on the observed locations. The idea is to use a kernel in order to scale the impact of observed locations in the densification process.

\subsection{Representation}

\subsubsection{Velocity}

A fundamental concept used in this chapter is velocity. Velocity is a vector quantity defined by direction and magnitude. In this chapter, velocity $\mathbf{V}$ will be described as a point in a polar coordinate frame, combining orientation $(\theta)$ and speed $(\rho)$ as in Eq. (4.1). In contrast to a representation using a $2 \mathrm{D}$ 
Euclidean vector $\left(\mathrm{V}_{\mathrm{x}}, \mathrm{V}_{\mathrm{y}}\right)$, in a polar representation each component has an explicit physical meaning and can be analysed independently.

$$
\mathbf{V}=(\theta, \rho)^{\top}, \rho \in \mathbb{R}^{+} \wedge \theta \in[0,2 \pi)
$$

\subsubsection{Semi-Wrapped Normal Distribution}

In order to model the variability of velocity, I use a Semi-Wrapped Normal Distribution (SWND), which combines direction - a circular variable, whose domain can be visualised as a circumference of the unit circle - with a speed which is a positive real number. We can imagine that such a distribution is a regular bivariate normal distribution wrapped around a cylinder, where one of the random variables is wrapped around the circumference of the cylinder while the other one runs along the length of it.

One of the methods to model probability density function (PDF) of a circular variable is to use a wrapped PDF. The wrapped PDF, in general, is a probability distribution describing data on an $n$-dimensional sphere. In one dimension (such as a planar motion's direction) a wrapped distribution consists of values on the unit circle and can be visualised as "wrapping" a continuous distribution around the circle. The "wrapping" of a distribution around a unit circle is defined as a periodic sum of period $2 \pi$. Thus, the PDF of a wrapped normal distribution $\left(\mathcal{N}_{\mu, \Sigma}^{\mathbb{W}}\right)$ is defined as:

$$
\mathcal{N}_{\mu, \Sigma}^{\mathbb{W}}(\theta)=\sum_{k \in \mathbb{Z}} \mathcal{N}_{\mu, \Sigma}(\theta+2 k \pi) \wedge \theta \in[0,2 \pi)
$$

where $\mu$ and $\Sigma$ denote mean value and variance of the distribution respectively. The winding number $k$ is an integer that represents the total number of times that the function has been wrapped around the circle.

The support of the normal distribution is all real numbers. Therefore in principle, it is necessary to wrap the distribution an infinite number of times around the unit circle, thus in Eq. (4.2) $k \in \mathbb{Z}$. However, Mardia and Jupp [47] comment that, for practical purposes, the density can be approximated by truncation to $k \in\{-1,0,1\}$ for cases where $\Sigma>2 \pi$, and $k=0$ for $\Sigma<2 \pi$. I will use this approximation to make the computations tractable.

The second component of velocity is speed $(\rho)$, that is a non-wrapped variable. A PDF of a SWND describing velocity, can be denoted as:

$$
\mathcal{N}_{\mu, \Sigma}^{\mathrm{SW}}(\mathbf{V})=\sum_{k \in \mathbb{Z}} \mathcal{N}_{\mu, \Sigma}\left(\left[\begin{array}{l}
\theta \\
\rho
\end{array}\right]+2 \pi\left[\begin{array}{l}
k \\
0
\end{array}\right]\right)
$$

where $\boldsymbol{\mu}$ and $\boldsymbol{\Sigma}$ denote mean value and covariance matrix of a heterogeneous quantity, which combines speed $(\rho)$ and orientation $(\theta)$. 


\subsubsection{Semi-Wrapped Gaussian Mixture Model}

In many cases dynamic phenomena such as wind or pedestrian flow are multimodal, namely, in any one location, it is possible to observe different motion patterns (i.e. different orientations, different speeds or both). In order to retain the multimodal characteristic of the flow, I use a SWGMM[65], which can be pictured as Gaussian Mixture Model (GMM) on a cylinder. Fig. 4.2 shows a visualisation of such a wrapped distribution with five modes.

Similarly to a GMM an SWGMM is a PDF represented as a weighted sum of J SWNDs

$$
p(\mathbf{V} \mid \boldsymbol{\xi})=\sum_{j=1}^{J} \pi_{j} \mathcal{N}_{\boldsymbol{\mu}_{j}, \Sigma_{j}}^{S W}(\mathbf{V}),
$$

where $\xi$ denotes a finite set of components of the SWGMM:

$$
\xi=\left\{\xi_{j}=\left(\boldsymbol{\Sigma}_{j}, \boldsymbol{\mu}_{j}, \pi_{j}\right) \mid j \in \mathbb{Z}^{+}\right\} .
$$

Each component $\xi_{j}$ is defined by its mean $\boldsymbol{\mu}_{j}$, covariance $\boldsymbol{\Sigma}_{j}$ and mixing factor $\pi_{j}$. To each component, there can be associated a physical interpretation. The means $\boldsymbol{\mu}_{j}$ define the dominant directions in a given location. The covariances $\Sigma_{j}$ describes the variability of observations contributing to dominant directions observed in a given location. Finally, the mixing factor $\pi_{j}$ represents how common that mode of motion is (relative to the other modes at the location).

\subsubsection{Motion Ratio and Observation Ratio}

The goal of the Circular Linear Flow Field map is to model motion patterns in space. In order to do that, CLiFF-map associates to locations in the map SWGMMs describing the motion patterns in them. However, using only SWGMMs to describe the motion patterns has two significant shortcomings.

First, they do not describe the intensity of the motion. Let us assume that there are two locations $l_{1}$ and $\boldsymbol{l}_{2}$ in the environment observed by the robot, both of which were observed for an hour each $\left(T_{0}^{1}=1 \mathrm{~h}\right.$ and $\left.\mathrm{T}_{\mathrm{o}}^{2}=1 \mathrm{~h}\right)$. The resulting SWGMMs are identical, but dynamics was observed for ten minutes $\left(\mathrm{T}_{\mathrm{m}}^{1}=10 \mathrm{~min}\right)$ in the first location while for fifty minutes $\left(\mathrm{T}_{\mathrm{m}}^{2}=50 \mathrm{~min}\right)$ in the second location. The difference in the intensity of dynamics is very useful information, which is not preserved in the SWGMM itself. In order to incorporate this information, CLiFF-map associates a motion ratio $\bar{p}_{k}$ to each location $l_{k}$. The motion ratio is the ratio of the duration during which motion has been observed $T_{m}$ and the total observation duration $T_{o}$ for location $l_{k}$ :

$$
\overline{\mathrm{p}}_{\mathrm{k}}=\frac{\mathrm{T}_{\mathrm{m}}}{\mathrm{T}_{\mathrm{o}}} \wedge\left(\mathrm{T}_{\mathrm{m}} \leqslant \mathrm{T}_{\mathrm{o}}\right) .
$$

The value of $\bar{p}$ depends on both the observation time $T_{0}$ and duration of observed motion $T_{m}$. 


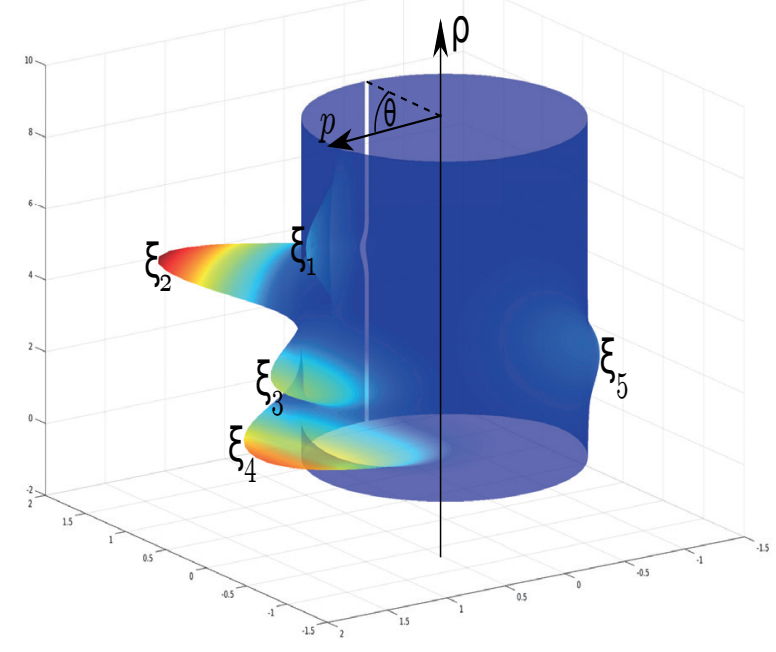

Figure 4.2: An example of SWGMM wrapped on a unit cylinder. In the figure, we can see a distribution with five modes $\left(\xi_{j}\right)$. The position along rho axis represents the velocity; furthermore, the orientation is denoted as $\theta$. Finally, the distance from the surface of the cylinder corresponds to the probability $p$.

In case of dynamic maps, the time spent on observation of a location can substantially affect the quality of a map. In order to quantify the relative amount of information obtained for each location I introduce observation ratio $\overline{\mathrm{q}}_{k}$, which is the ratio between the total observation duration $T_{0}$ of a given location and maximum observation duration for any location $T_{t}$ :

$$
\overline{\mathrm{q}}_{\mathrm{k}}=\frac{\mathrm{T}_{\mathrm{o}}}{\mathrm{T}_{\mathrm{t}}} .
$$

A high $\bar{q}_{k}$ value means that given location was observed for a substantial amount of time. Therefore, the model for such a location should be more reliable than for a location with a lower observation ratio.

In summary, the CLiFF-map is defined as a set of SWGMMs coupled with their locations $\left(\boldsymbol{l}_{k}\right)$, motion ratio $\left(\overline{\mathrm{p}}_{\mathrm{k}}\right)$ and observation ratio $\left(\overline{\mathrm{q}}_{\mathrm{k}}\right)$, denoted as

$$
\Xi=\left\{\left(\boldsymbol{\xi}_{k}, \overline{\mathrm{p}}_{\mathrm{k}}, \overline{\mathrm{q}}_{\mathrm{k}}, \boldsymbol{l}_{\mathrm{k}}\right) \mid \mathrm{k} \in \mathbb{Z}^{+} \wedge \boldsymbol{l}_{\mathrm{k}} \in \mathbb{R}^{2}\right\} .
$$




\subsection{Map Building}

In this section, I will describe the process of building a CLiFF-map. In the map building process (see Fig. 4.1), to each of the observed locations based on the collected measurements there is associated a GMM describing the local velocity model. The building of a CLiFF-map can be described as an unsupervised model fitting for multiple data sets.

The core of the method is to use Expectation Maximisation (EM) (see Sec. 4.3.4) to estimate the parameters of the SWGMM for each location. In order to use EM it is necessary to provide a set of initial estimates of the model parameters. That is the number of clusters, and initially estimated means, covariances and mixing factors (see Sec. 4.3.3). I estimate the initial conditions using clustering algorithms.

\subsubsection{Data Discretisation}

CLiFF-map builds a map of a flow field as if all the observations were obtained at a discrete set of measurement locations $\left(\mathbf{l}_{1 . . \mathrm{K}}\right)$. For wind mapping, where the velocities are measured in situ, such locations would be places where the robot stopped to acquire data. In contrast, measures of people's velocities etc., where measurements are obtained with range sensor: each measurement is associated with a different position in the map. When creating a CLiFF-map, measurements are aggregated at a discrete set of locations, by assuming that each measurement within radius $r$ of $\boldsymbol{l}_{k}$ was in fact obtained at $\boldsymbol{l}_{k}$.

In Fig. 4.3 we can see a visualisation of the way this procedure works. All velocity observations inside the blue circle are associated with its centre. This procedure is repeated for each location.

In Fig. 4.3 locations are placed on a regular grid. However, the method is not limited only to grid-based spatial discretisation. The discretisation can also be achieved by dividing the area into arbitrary sub-areas. In this case, the dynamics of each sub-area, despite the shape and size, will be described by a single SWGMM.

\subsubsection{Mathematical Operations in Circular-Linear Space}

As I have mentioned in Sec 4.2.1 velocity is a heterogeneous quantity combining circular and linear values. In this section, I present how to account for this heterogeneity in basic mathematical operations, used in the following part of this chapter. 


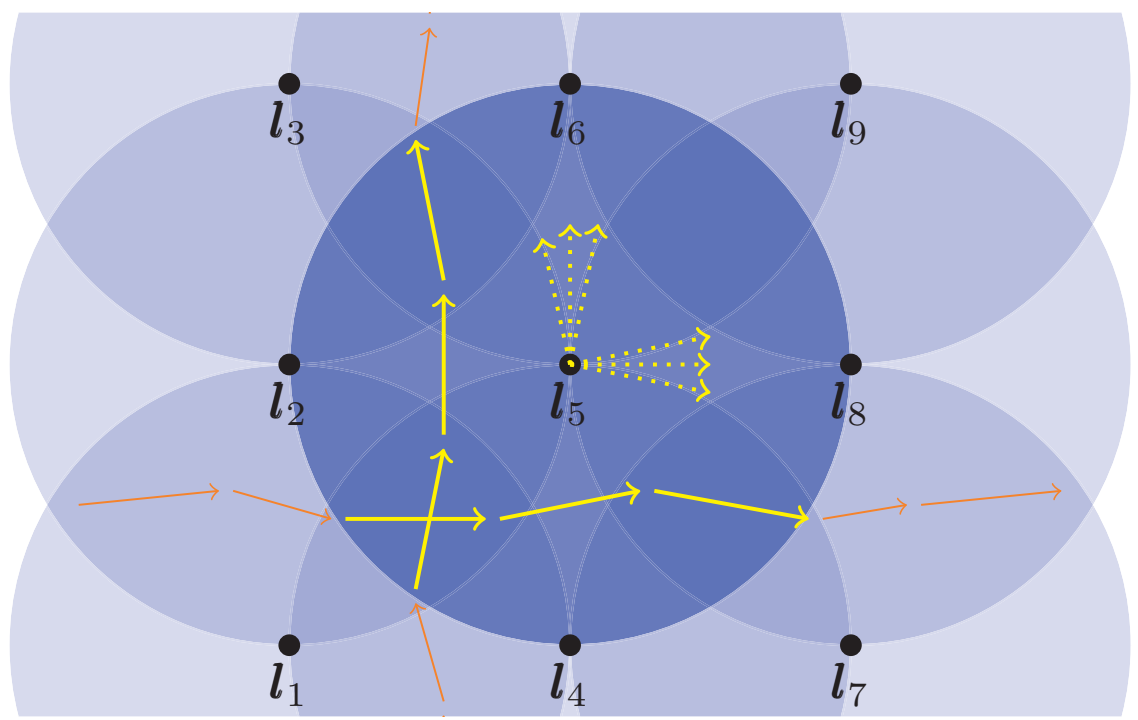

Figure 4.3: Measurement discretisation procedure. Each blue circle represents an area with radius $r$ from which measurements are associated with the location in its centre. Yellow arrows represent the measurements that are associated with location $\mathfrak{l}_{5}$. The orange arrows represent measurements not taken into account for $\mathbf{l}_{5}$.

\section{Circular-Linear distance}

The first problem to be addressed is a computation of the distance between two points in Circular-Linear (C-L) space. Roy and Puri [65] suggest to use a distance defined as follow:

$$
\mathrm{d}^{\mathrm{c}}\left(\mathbf{V}_{1}, \mathbf{V}_{2}\right)=1-\cos \left(\theta_{1}-\theta_{2}\right)+\left|\rho_{1}-\rho_{2}\right|
$$

However, the formula presented in Eq. (4.9) leads to a situation when the difference in orientation has a lower impact than the difference in speed. In Fig. 4.4a we can see a visualisation of the distance function.

To increase the impact of orientation difference on the distance metrics, I suggest using a modified version of Euclidean distance $d^{e}$ in C-L space.

$$
\mathrm{d}^{e}\left(\mathbf{V}_{1}, \mathbf{V}_{2}\right)=\sqrt{w\left(\theta_{1}-\theta_{2}\right)^{2}+\left(\rho_{1}-\rho_{2}\right)^{2}}
$$

In Eq. $4.10 \mathrm{I}$ assume that the angular distance between two points in C-L space is equal to the smallest angle between them and is limited to the interval from 0 to $\pi$ : 


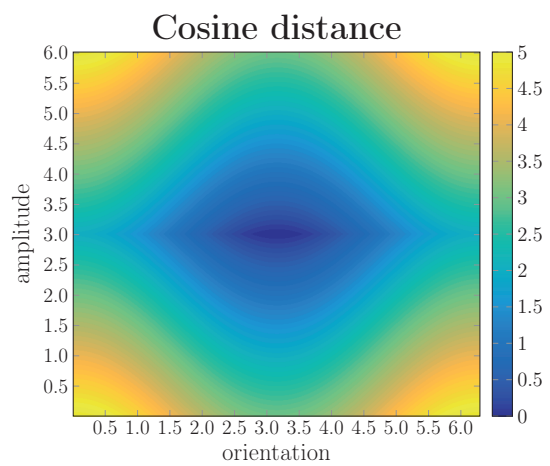

(a) Visualisation of cosine distance between the point in the centre $(3,3)$ and all the points within an interval $\{(\theta, \rho) \mid 0 \leqslant$ $\theta \leqslant 2 \pi \wedge 0 \leqslant \rho \leqslant 6\}$. We can see that the angular differences are affecting the overall metrics less then the differences in amplitude.

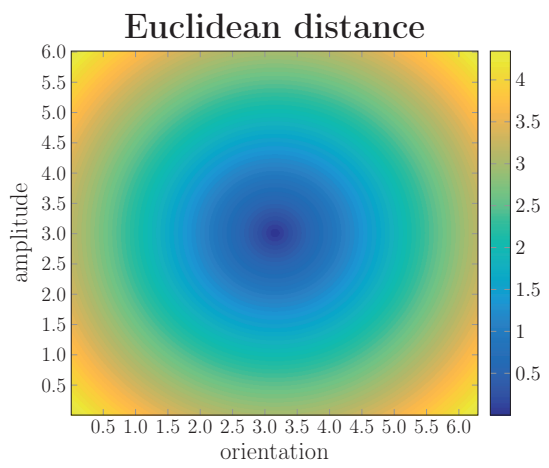

(b) Visualisation of Euclidean distance between the point in the centre $(3,3)$ and all the points within an interval $\{(\theta, \rho) \mid 0 \leqslant$ $\theta \leqslant 2 \pi \wedge 0 \leqslant \rho \leqslant 6\}$. We can see that the angular distances are affecting the overall metrics more than in the case of cosine distance (see Fig. 4.4a).

Figure 4.4: Comparison of different distance metrics.

$$
\mathcal{w}(\theta)= \begin{cases}|(\theta+\pi)(\bmod 2 \pi)-\pi| & \text { if }(\theta<-\pi) \vee(\theta>\pi) \\ |\theta| & \text { otherwise }\end{cases}
$$

In $4.4 \mathrm{~b}$ we can see that the proposed modification of Euclidean distance results in a higher overall impact in distance measurements for small angular changes. The proposed distance metrics also has limitations, namely, for substantial differences in the speed, the orientation differences become negligible.

\section{Circular-Linear mean}

Another fundamental computation used through the remainder of this chapter is a C-L mean In this work, I am combining a linear mean with angular mean as defined by Mardia and Jupp in [47].

Let us denote the matrix containing all observations $\left(\mathbf{V}_{n}\right)$ as $\boldsymbol{A}_{\mathbf{N} \times 2}$.

$$
\boldsymbol{A}_{\mathrm{N} \times 2}=\left[\begin{array}{cc}
\theta_{1} & \rho_{1} \\
\theta_{2} & \rho_{2} \\
\vdots \\
\theta_{\mathrm{N}} & \rho_{\mathrm{N}}
\end{array}\right]
$$

The size of the matrix is $\mathrm{N} \times 2$, where $\mathrm{N}$ is the number of observations. 
The mean value $\mathbf{G}$ of the set of observations consist of mean orientation $(\bar{\theta})$ and mean speed $(\bar{\rho})$ :

$$
\mathrm{G}=\left[\begin{array}{l}
\bar{\theta} \\
\bar{\rho}
\end{array}\right]
$$

The computation of a mean orientation $(\bar{\theta})$ comprises two steps. In the first step, a mean of sine values $\bar{S}$ and mean of cosine values $\bar{C}$ for the orientations $\theta_{\mathrm{n}}$ is computed. In the second step, the arctangent of $\bar{S}$ and $\bar{C}$ is computed in order to retrieve mean orientation.

$$
\begin{gathered}
\bar{S}=\frac{1}{N} \sum_{n=1}^{N} \sin \theta_{n} \\
\bar{C}=\frac{1}{N} \sum_{n=1}^{N} \cos \theta_{n} \\
\bar{\theta}= \begin{cases}\operatorname{atan}(\bar{S}, \bar{C}) & \text { if } \bar{C} \geqslant 0 \\
\operatorname{atan}(\bar{S}, \bar{C})+\pi & \text { if } \bar{C}<0\end{cases}
\end{gathered}
$$

The mean speed $\bar{\rho}$ is computed as the arithmetic means of all the observed speeds $\rho_{n}$ :

$$
\bar{\rho}=\frac{1}{N} \sum_{n=1}^{N} \rho_{n}
$$

\subsubsection{Clustering}

The core element of the mapping system is the EM algorithm used for estimation of parameters of SWGMM in each of the locations. However EM requires a set of initial parameters. One of the methods to provide such an initial guess is to use a clustering algorithm to obtain a set of clusters. Further, the parameters of each cluster are used as a prior. In this section, I will discuss three different clustering algorithms (Mean Shift (MS), k-means, Ordering points to identify the clustering structure (OPTICS)), and discuss their qualities when used to obtain the initial guess of the parameters for EM.

Let $\mathbf{P}_{\mathrm{N} \times 1}$ denote a partition of a data set. A partition is a vector containing numeric labels for each data entry in $\boldsymbol{A}_{\mathrm{N} \times 2}$. Therefore cluster $C_{k}$ is a submatrix $\boldsymbol{A}_{N_{k} \times 2}^{\{k\}}$, where $N_{k}$ denotes the number of rows in the submatrix and also the number of elements in a cluster $C_{k}$ and $k$ denotes cluster's label.

$$
\mathrm{C}_{\mathrm{k}}=\left\{\left(\theta_{i}, \rho_{\mathrm{i}}\right) \mid \mathrm{P}_{\mathrm{i}}=\mathrm{k}\right\}
$$

The centroid of the cluster $C_{k}$ can be then computed as shown in Eq. (4.16) and is denoted as:

$$
G_{k}=\left[\begin{array}{l}
\bar{\theta}_{k} \\
\bar{\rho}_{k}
\end{array}\right]
$$




\section{Mean Shift}

Mean Shift (MS) [19] is a mode seeking algorithm that can be used to obtain the number and initial positions of modes and corresponding covariances used by the EM. MS is a hill-climbing algorithm. The hill-climbing algorithms are iterative algorithms that start with an arbitrary solution which later on is improved by changing the solution. The procedure continues until the further improvement is not possible. The intuition behind MS is to "shift" the data points to the location with the highest density.

Let an initial estimate $\mathbf{V}$ and a set of velocity measurements $\boldsymbol{A}=\left\{\mathbf{V}_{1}, \ldots, \mathbf{V}_{\mathbf{N}}\right\}$ be given. Let a kernel function $\mathrm{K}\left(\mathbf{V}_{i}-\mathbf{V}\right)$ be given. The kernel function determines the weight of nearby points for re-estimation of the mean. The weighted mean is then computed as follows:

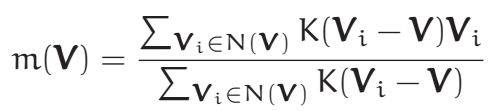

where $\mathbf{N}(\mathbf{V})$ is the neighbourhood of $\mathbf{V}$. The MS in each iteration sets $\mathbf{V} \leftarrow$ $\mathrm{m}(\mathbf{V})$ until $\mathrm{m}(\mathbf{V})$ converges.

The key element of MS is the kernel which shape and size determine the behaviour of the algorithm. In this work, I use two types of the kernel: isotropic and non-isotropic Gaussian kernel. The isotropic kernel treats the angular and linear distance jointly, while the non-isotropic kernel is considering the angular and linear distance separately.

Isotropic Kernel I define the window as an isotropic Gaussian whose bandwidth is estimated with Silverman's rule [70]:

$$
\sigma=\left(\frac{4 \hat{\sigma}^{5}}{3 \mathrm{~N}}\right)^{\frac{1}{5}}
$$

where $\hat{\sigma}$ is the standard deviation of the samples and $N$ is the number of samples for the whole data set. Silverman's rule was originally defined for uni-variate data [69]; however, velocity measurements are inherently bi-variate data. In order to apply Silverman's rule, I express each point as its distance to the origin of the coordinate frame. In Eq. (4.10) I have defined a distance between two points in the CL-space. In this case, the distance from the origin of the coordinate frame Eq. (4.10) will look as follows:

$$
\mathrm{d}_{e}(\mathbf{V})=\sqrt{w(\theta)^{2}+(v)^{2}}
$$

In this way we can project the two dimensional data to one dimension, and compute the kernel size in the distance space. 
Non-Isotropic Kernel In order to reflect the heterogeneity of velocity data, I will also use a non-isotropic kernel in the evaluation process. To build a nonisotropic kernel, I employ Silverman's rule twice, along each of the dimensions separately $\left(\sigma_{\theta}\right.$ for orientation and $\sigma_{\rho}$ for speed), and then combine the result in the covariance matrix:

$$
\Sigma=\left[\begin{array}{cc}
\sigma_{\theta} & 0 \\
0 & \sigma_{\rho}
\end{array}\right]
$$

\section{k-means}

Another considered clustering method is k-means introduced by Macqueen [44]. This algorithm aims to break an $\mathrm{N}$-element population into $\mathrm{k}$ sets. The algorithm can be defined as minimisation of the sum of the distances from the centroid $\mathrm{G}_{\mathrm{k}}$ to the cluster members.

To perform clustering with $\mathrm{k}$-means it is required to have a set of initial centroids $G_{k}$. In original work, those centroids are randomly picked points from the dataset. Arthur and Vassilvitskii in [4] presented an improved method for seeding the initial positions of the cluster centres called k-means++, which improves not only the speed but also the accuracy of the clustering. Even though $\mathrm{k}$-means++ provides an improved method to pick the initial location of clusters, it does not tell how many clusters there should be.

In order to estimate the number of clusters in the considered data, I run the clustering procedure with a different number of clusters. Each of the results is evaluated with Davies-Bouldin Index (DBI) [20]. Out of the resulting set, I pick the one with the best DBI score.

In the section considering MS, I have discussed how two ways of tackling the problem of heterogeneity of the data. For k-means, a similar modification can be included. Namely, the shape of the kernel in MS can be translated to a modification of the distance function in k-means. The isotropic kernel is equivalent to the unmodified distance function as shown in Eq. (4.10), while the non-isotropic kernel is equivalent to a weighted distance:

$$
\mathrm{d}_{\mathrm{e}}^{w}\left(\mathbf{V}_{1}, \mathbf{V}_{2}\right)=\sqrt{\frac{\sigma_{\theta}}{\sigma_{\rho}} \mathcal{w}\left(\theta_{i}-\theta_{j}\right)^{2}+\left(\rho_{i}-\rho_{j}\right)^{2}}
$$

In Eq. (4.24) the weight is applied to the angular distance. The weight is computed as the ratio between the standard deviation of orientation $\sigma_{\theta}$ standard deviation of speed $\sigma_{\rho}$. Both quantities computed according to Eq. (4.21).

\section{OPTICS}

OPTICS (Ordering points to identify the clustering structure) developed by Ankerst et al. in [2] is a method for density clustering. In principle, this method can be treated as a generalisation of DBSCAN (Density-based spatial clustering 


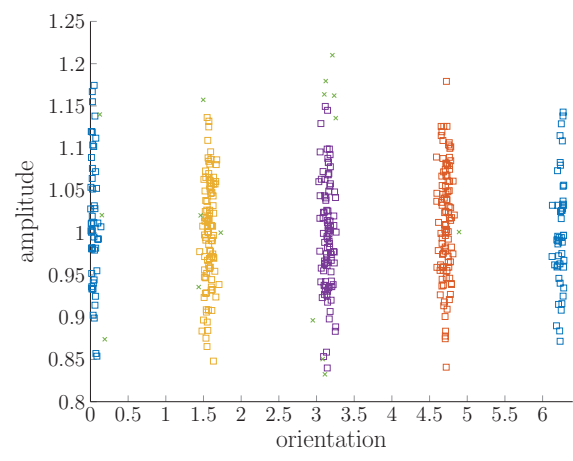

(a) Plot of clusters.

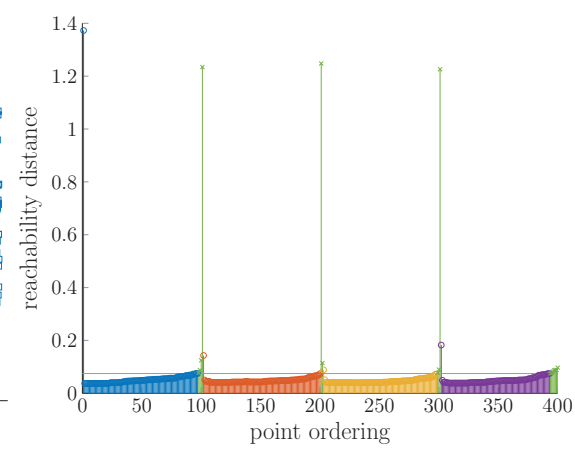

(b) Reachability plot.

Figure 4.5: Example of clustering of a simple simulated data set with OPTICS. The colours on the cluster plot are corresponding to the colour on reachability plot.

of applications with noise) propose by Ester et al. in [24]. OPTICS linearly orders points in the data set such that the points that are spatially closest become neighbours in the ordering (see Fig. 4.5b). Furthermore, to each point in the ordering, there is associated a reachability distance. Intuitively, the reachability distance can be understood as a measure of density around the considered point.

Once the reachability plot is obtained clusters can be extracted from it. The density clustering means that the algorithm should look for groups of points close to each other (with low reachability distance). Such groups of points close to each other are visible as dents. Therefore, the cluster extraction is formulated as detection of such dents and identifying points belonging to each one of them. Finally, if the reachability distance for a point is too exceeds the maximum radius of the neighbourhood, then such a point is treated as noise (green points in Fig. 4.5a and Fig. 4.5b) and not assigned to any cluster.

Similarly as for MS and k-means $(\mathrm{km})$, I have also evaluated the impact of different distance metrics on OPTICS clustering. In the evaluation section I analyse how (4.10) and (4.24) affect the quality of fitting.

\subsubsection{Fitting with Expectation Maximisation algorithm}

To estimate the parameters of the SWGMM, after initialising the parameters using one of the methods discussed in Sec. 4.3.3, the EM algorithm is used [22]. The general derivation of the update rules for circular-linear data was presented in the work of Roy and Puri [65]. They address a more general problem for the $n$-dimensional case. In my work, I focus on the 2D case for which the update rules look as follows. 


\section{Expectation Step}

$$
\eta_{i j k}^{\mathrm{t}}=\frac{\pi_{j}^{\mathrm{t}-1} \mathcal{N}\left(\mathbf{v}_{i} ; \boldsymbol{\mu}_{j}^{\mathrm{t}-1}+2 \pi\left[\begin{array}{l}
k \\
0
\end{array}\right], \Sigma_{j}^{\mathrm{t}-1}\right)}{\sum_{j=1}^{M} \sum_{k=-\infty}^{\infty} \pi_{j}^{\mathrm{t}-1} \mathcal{N}\left(\mathbf{v}_{i} ; \boldsymbol{\mu}_{j}^{\mathrm{t}-1}+2 \pi\left[\begin{array}{l}
k \\
0
\end{array}\right], \Sigma_{j}^{\mathrm{t}-1}\right)}
$$

In the expectation step, I compute the responsibility $\eta$ that cluster $j$ takes for the ith data point for the kth round of wrapping, based on the parameters estimated in the previous iteration of the algorithm.

Maximisation step In the maximisation step, I compute the new set of parameters $\Xi$ using the following update rules.

$$
\begin{gathered}
\pi_{j}^{t}=\frac{1}{N} \sum_{i=1}^{N} \sum_{k=-\infty}^{\infty} \eta_{i j k}^{t} \\
\boldsymbol{\mu}_{j}^{t}=\frac{\sum_{i=1}^{N} \sum_{k=-\infty}^{\infty}\left(\mathbf{v}_{i}-2 \pi\left[\begin{array}{l}
k \\
0
\end{array}\right]\right) \eta_{i j k}^{t}}{\sum_{i=1}^{N} \sum_{k=-\infty}^{\infty} \eta_{i j k}^{t}} \\
\Sigma_{j}^{t}=\frac{\sum_{i=1}^{N} \sum_{k=-\infty}^{\infty}\left(\mathbf{v}_{i}-\mu_{j}-2 \pi\left[\begin{array}{l}
k \\
0
\end{array}\right]\right)\left(\mathbf{v}_{i}-\mu_{j}-2 \pi\left[\begin{array}{l}
k \\
0
\end{array}\right]\right)^{\top} \eta_{i j k}^{t}}{\sum_{i=1}^{N} \sum_{k=-\infty}^{\infty} \eta_{i j k}^{t}}
\end{gathered}
$$

\subsubsection{Ridgeline analysis}

One of the problems that might occur in the process of model fitting is a redundant number of clusters provided by the clustering algorithm. An example of such a case is in Fig. 4.6. In order to address this problem, I have implemented a cluster pruning method based on ridgeline analysis described by Ray and Lindsay in [61].

Ray and Lindsay proposed a method for analysing the shape and mutual relation between the components of the multimodal distribution, through ridge line analysis. A ridge-line is a manifold containing all the critical points (modes, antimodes and saddle-points) as well as the ridges of the PDF (red line in Fig. 4.6).

In my work, I propose to use ridge lines to evaluate if the modes are significant enough to contribute to the overall SWGMM. Based on this results the redundant clusters can be pruned.

The pruning procedure is composed of following four steps: 


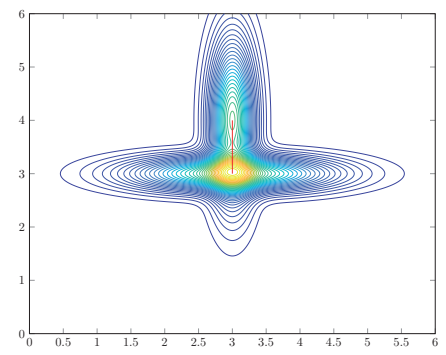

(a)

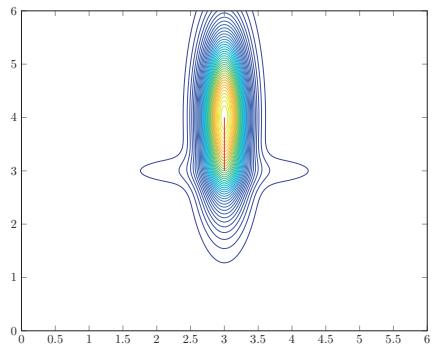

(c)

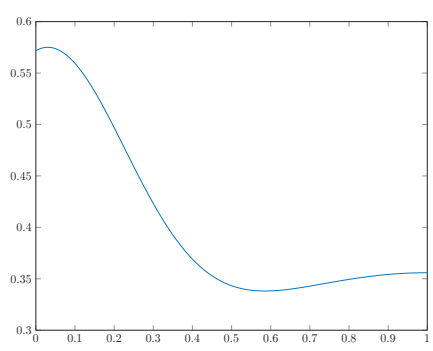

(b)

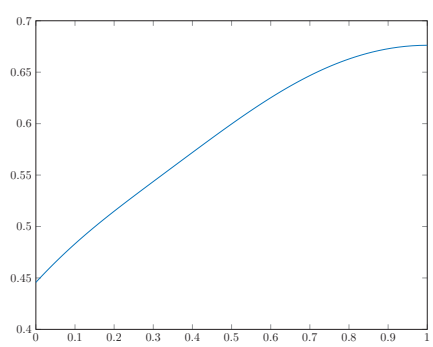

(d)

Figure 4.6: Figures a and c present two examples of the relation between two distributions constructing a SWGMM. In Figigure a the distributions are prominent, and the elevation plot (Fig. c) of the ridgeline (red line in Fig. a) has two maxima. In contrast, the elevation plot in Fig. $d$ shows that one of the distributions in Fig. $c$ is hidden with the other distribution. In the case shown in Fig. a the less prominent mode will not be removed in the case shown in Fig. $c$ the less prominent node will be removed, and the parameters of the distribution will be recomputed.

1. Compute the ridge-lines - In the first step I compute a pairwise ridge-lines for all the modes in the SWGMM.

$$
\boldsymbol{X}_{i, j}^{*}(\alpha)=\left[\bar{\alpha} \Sigma_{i}^{-1}+\alpha \Sigma_{j}^{-1}\right]^{-1}\left[\bar{\alpha} \Sigma_{i}^{-1} \boldsymbol{\mu}_{i}+\alpha \Sigma_{j}^{-1} \boldsymbol{\mu}_{j}\right],
$$

where

$$
\bar{\alpha}=1-\alpha, \alpha \in[0,1] .
$$

2. Analyse the ridge-lines - The basic tool to analyse the ridge-line is the ridge-line elevation plot.

$$
h(L(\alpha))=g\left(X_{i, j}^{*}(L(\alpha))\right),
$$

where $L(\alpha)$ is a length of an arch between the two modes and

$$
g(\mathbf{X})=\pi_{i} \mathcal{N}\left(\mathbf{X}: \boldsymbol{\mu}_{i}, \Sigma_{i}\right)+\pi_{j} \mathcal{N}\left(\mathbf{X}: \boldsymbol{\mu}_{j}, \Sigma_{j}\right) .
$$


By analysing the elevation plot we can compute how many extremes are present. This step is performed for each pair of the distributions. The general idea is to check if the elevation plot is monotonically descending from the higher to the lower peak. If the answer is yes, I assume that the lower peak is redundant and can be removed.

3. Remove redundant clusters In this step, a new set of the initial parameters for EM is computed.

If one of the distributions in the pair is found redundant in place of the pair a new single distribution is inserted. The mean of the new distribution is a weighted mean of the distributions in the pair. The new mixing factor is a sum of mixing factors of the two distributions of the pair.

$$
\begin{array}{r}
\mu_{i}^{n}=\frac{\pi_{i} \mu_{i}+\pi_{j} \mu_{j}}{\pi_{i}+\pi_{j}} \\
\pi_{i}^{n}=\pi_{i}+\pi_{j}
\end{array}
$$

The covariance matrix of a new distribution is equal to the covariance matrix of the more prominent distribution.

4. Recompute parameters of SWGMM Using the parameters obtained in the previous step, I restart the EM and repeat the procedure until no redundant clusters are detected.

It is important to remember the limitations of this method. The method is analysing each pair of modes separately; therefore it does not consider the influence of other modes during the analysis. This might lead to unnecessary removals of some modes. The result of an unnecessary removal of modes by ridgeline analysis can be observed as an increase of divergence between the underlying data and the model (see Sec. 4.6.2.)

\subsection{Map Densification}

In the previous section, I have discussed the problem of estimating parameters of SWGMM for locations where the measurements were collected. However, in some cases, the available data might be spatially too sparse to produce a dense map. In some cases, the available data might be spatially too sparse to produce a dense map.

In Fig. 4.1, I have shown that a CLiFF-map cannot only be built out of direct measurements but also it is possible to build a dense map out of sparse measurement locations. In such a case the parameters of SWGMM in unobserved location are estimated based on the observed locations.

Let us consider a scenario where a robot, equipped with an anemometer, is patrolling an environment and collecting wind measurements in arbitrary locations. In Fig. 4.7 we can see an example of a path followed by a robot 


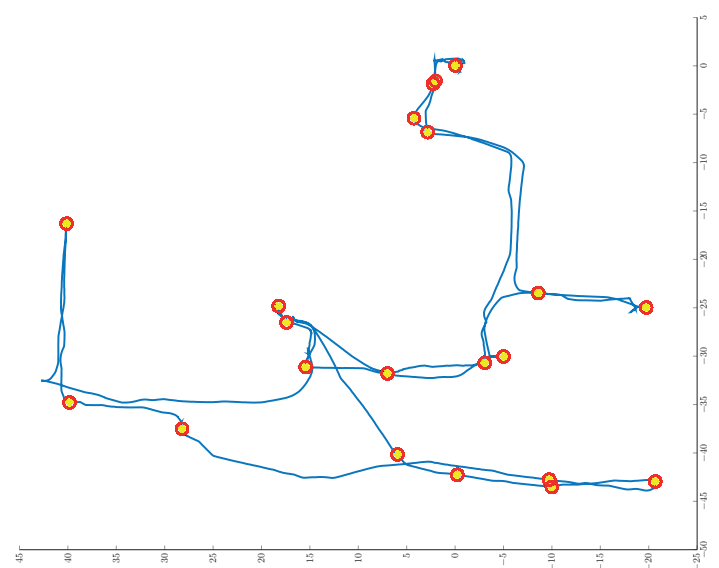

Figure 4.7: In this figure we can see a path of a robot during a data collection campaign. The red and yellow markers mark the locations in which the measurements were obtained.

while collecting the data. The red-yellow markers label the locations where the air flow measurements were obtained. In this scenario, a goal is to build a complete map of air flow based on just a few measurement locations.

The process of map densification can be referred to as missing-data imputation. The imputation is a process where based on the existing data missing data is generated. In my work I have compared two imputation methods: Monte Carlo (MC) Imputation and Nadaraya Watson (NW) Imputation.

MC imputation in each step draws one of the existing models with probability inversely proportional to the distance between the observed location and unobserved location. Then from the picked location, a virtual observation is sampled according to the SWGMM.

In contrast, NW imputation does not add a virtual observation drawn from one of the observed locations but instead, it computes a weighted mean of the samples drawn from all observed locations. Then, the computed mean is added as a new virtual observation in the unobserved location.

In both methods, once enough observations are collected parameters, the of SWGMM for the unobserved location are estimated using EM. Examples of estimated distributions with MC and NW imputation are shown in Fig. 4.8. Monte Carlo (MC) Imputation tends to preserve multimodal characteristics of the data and keep the sharp borders between different motion directions, while Nadaraya Watson (NW) Imputation smooths the data and introduces gradual changes. 


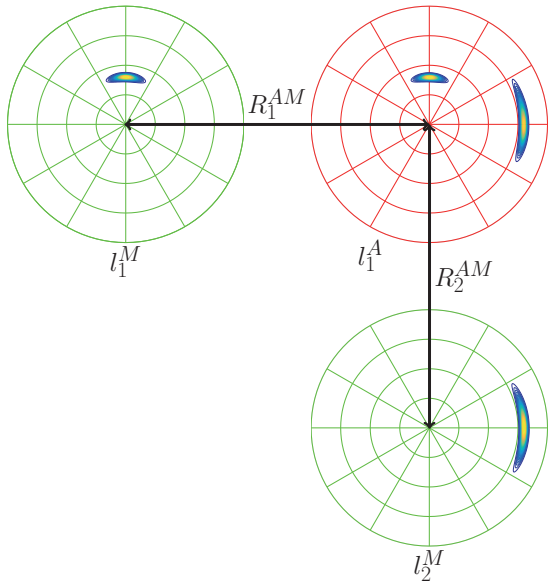

(a) MC imputation.

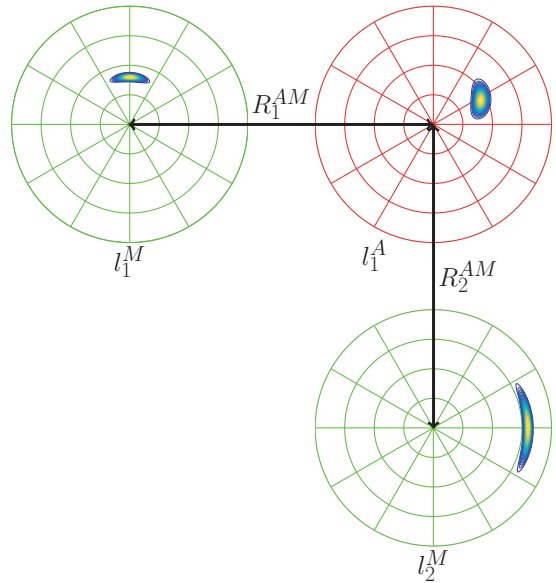

(b) NW imputation.

Figure 4.8: Comparison of imputation methods. Green plots describe the SWGMM for observed locations $\left(l_{1}^{M}, l_{2}^{M}\right)$, and red is an unobserved location $\left(l_{1}^{A}\right)$. The distances between the observed and unobserved locations are denoted as $R_{1}$ and $R_{2}$

\subsubsection{Monte Carlo Imputation}

This method relies on sampling virtual observations from the surrounding, already learned, distributions (Fig 4.8a). In order to obtain virtual observations, a hierarchical sampling is used.

The first step is to pick one of the existing SWGMMs $\left(\boldsymbol{\xi}_{i}\right)$. The likelihood of picking an SWGMM $\xi_{i}$ is proportional to the distance $\left(R_{i}^{A M}\right)$ between the location to be estimated $\left(\mathbf{l}_{1}^{A}\right)$ and the existing one $\left(\mathbf{l}_{i}^{M}\right)$ and also proportional to observation ratio $\overline{\mathrm{q}}_{i}$ :

$$
\begin{gathered}
R_{i}^{A M}=\left|\mathbf{l}_{1}^{A}-\mathbf{l}_{i}^{M}\right| \\
p\left(\xi=\xi_{i} \mid \bar{q}_{i}, K\left(R_{i}^{A M}\right)\right)=\bar{q}_{i} K\left(R_{i}^{A M}\right)
\end{gathered}
$$

In Eq. (4.36), $\mathrm{K}(\bullet)$ is a kernel function defining the size and shape of the sampling window. In this work, a zero-mean Gaussian kernel is used:

$$
K\left(R_{i}^{A M}\right)=\mathcal{N}\left(R_{i}^{A M} \mid 0, \sigma\right) .
$$

The second step is to sample a virtual observation from the SWGMM. There can be two results of sampling a virtual observation, depending on the value describing the motion ratio $\left(\bar{p}_{i}\right)$. It can be either a virtual velocity $(\hat{\mathbf{V}}=(\theta, \rho))$ or an empty observation. 
The virtual velocity is sampled from the SWGMM describing the motion pattern in a location picked in the previous step. The probability of sampling a virtual velocity $(\hat{\mathbf{V}})$ is equal to:

$$
p\left(\mathbf{V}=\hat{\mathbf{V}} \mid \bar{q}_{i}, \xi_{i}\right)=\bar{q}_{i} \sum_{j=1}^{J} \mathcal{N}^{S W}\left(\hat{\mathbf{V}} \mid \boldsymbol{\mu}_{j}, \Sigma_{j}\right) \pi_{j}
$$

The empty observations allow to on the one hand to propagate the information about the intensity of the motion and on the other hand lower the impact of locations with very few observations on the general estimation of the motion pattern.

\subsubsection{Nadaraya Watson Imputation}

NW imputation also relies on sampling virtual observations. However, in contrast to MC imputation, this time virtual observations are sampled from all observed locations on the map weighted by a kernel $\mathrm{K}(\bullet)$.

The realisation $\hat{\mathbf{V}}$ from each SWGMM is computed in this same way as in MC imputation (see Eq. (4.38)). Once virtual velocities are collected the weighted mean is computed $\overline{\mathbf{V}}$. The weights are proportional to the distance and the observation ratio as shown in Eq. (4.39).

$$
\overline{\mathbf{V}}=\frac{\sum_{i=1}^{N} K\left(R_{i}^{A M} \bar{q}_{i}^{-1}\right) \hat{\mathbf{V}}_{i}}{\sum_{i=1}^{N} K\left(R_{i}^{A M} \bar{q}_{i}^{-1}\right)}
$$

\subsubsection{Trust Estimation}

In Sec. 4.2.4 I have introduced motion ratio and observation ratio. These two factors in an implicit way describe our confidence in the estimated motion models. This concept is founded on the fact that for many practical applications, the greater number of observations help in improving the quality of the model parameters estimation and the predictive capabilities.

The observation ratio represent the relative time a given location was observed. The intuition of using this ratio to measure the confidence stems from the assumption that the longer a location is observed, the more information is collected thus the confidence in the quality of the model in the location is higher.

In CLiFF-map the focus is on modelling the motion patterns using a SWGMM to describe local velocity distribution. Therefore I assume that if the SWGMM describing the motion pattern in a given location is more trustworthy if it is supported by a greater number of observations.

In the densification process, the locations are not observed, but the desired number of samples is obtained from the surrounding, observed locations. In such a case the metrics introduced in Sec. 4.2.4 are not valid anymore. 
In order to address this problem, I introduce a trust factor $\hat{\mathrm{t}}$, which estimates the trust in the quality of the dense map. In the run of my work, I have devised two methods to estimate its value.

The first method used to estimate the trust factor builds on the idea that the trust factor should reflect the distance between the observed and estimated locations and the number of non-empty observations. The intuition behind this idea is that the shorter the distance to observed location and the more virtual observations supporting SWGMM, the higher the trust in the estimated distribution. However, this idea does not include the trust for already observed locations. Relying only on the distance to observed location can lead to the following situation. Let us consider two locations, the first one is near the observed location with low trust factor and the other one is equally close to the observed location with high trust. In this case, both of the new locations will get this same trust score. In order to address this issue, I have decided to use a Gaussian Process instead. Using Gaussian Process (GP) allows to incorporate the information about the trust of observed locations and also impact of a distance on the trust estimate for the unobserved location.

\section{Trust Estimation with Weighted Mean}

The first approach was to estimate the trust factor for each estimated location is based on the distance to the locations contributing to the estimated location. Depending on the chosen imputation method these factors are computed in different ways.

Trust for MC The intuition behind this factor is that we have more trust in estimated locations which are closer to observed ones.

$$
\hat{\mathrm{t}}=\frac{M}{\sum_{i=1}^{N}\left(R_{i}^{\text {AM }}+1\right)},
$$

where $\mathrm{N}$ is total number of virtual observations, including the empty observations, $M$ is the number of realisations of $\mathbf{V}$ and $R_{i}^{A M}$ are the distances between new location and the SWGMM selected in step 1.

Trust for NW For this imputation method, I compute the trust factor as the number of observations having a high impact on a new location, divided by the distance from $\mathbf{l}_{1}^{\mathrm{A}}$,

$$
\hat{\mathrm{t}}=\frac{\sum_{i=1}^{N} I\left(K\left(R_{i}^{\text {AM }}\right)\right)}{\sum_{i=1}^{N} I\left(K\left(R_{i}^{\text {AM }}\right)\right) R_{i}^{\text {AM }}+I\left(K\left(R_{i}^{\text {AM }}\right)\right)},
$$

where $\mathrm{I}(\bullet)$ is an indicator function

$$
I(d)= \begin{cases}1 & \text { if } d<3 \sigma \\ 0 & \text { if } d \geqslant 3 \sigma\end{cases}
$$




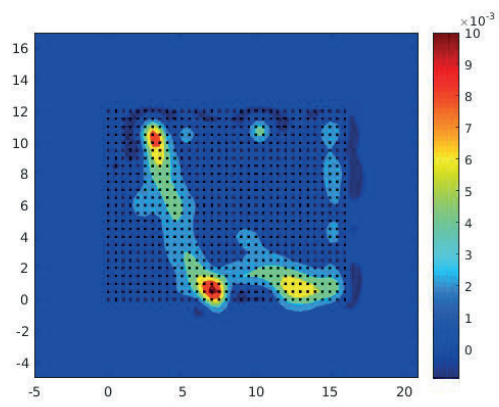

(a) Mean value of GP.

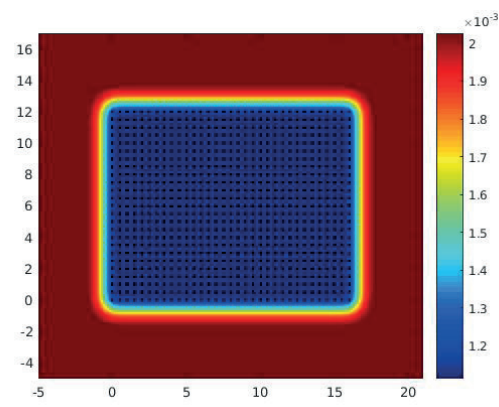

(b) Variance of GP

Figure 4.9: Visualisation of Gaussian Process representing trust factor based on complete set of observations. The black dots mark locations of SWGMM on the map.

\section{Trust estimation with Gaussian Process}

The method presented in the previous section compute the trust based only on distance; however in Sec. 4.2.4 I have introduced motion ratio $\overline{\mathrm{p}}_{j}$ and observation ratio $\overline{\mathrm{q}}_{j}$. These two parameters denote the trust in the existing distribution. Therefore in order to retain the information provided by these two parameters and also include the information about the distance between the estimated and observed locations I have introduced a method for trust factor estimation( $(\hat{\mathrm{t}})$ with a Gaussian Process.

First, to each observed location (at the position denoted as $(x, y)$ ) a trust coefficient is assigned:

$$
\mathrm{t}_{(x, y)}=\overline{\mathrm{p}}_{(x, y)} \overline{\mathrm{q}}_{(x, y)}
$$

These values are used as training data set for a GP.

Fig. 4.9 shows the mean values and variance for a complete CLiFF-map for one day in Edinburgh dataset ${ }^{1}$ [45]. In this scenario, all of the locations were observed for this same amount of time, and the value of the trust factor depends only on the intensity of the flow. In Fig. $4.9 \mathrm{~b}$ we can see the value of the variance for this GP. We can see that for the central area the value of the variance is low, while for the unmapped are the variance grows rapidly. Intuitively, the value of the variance corresponds to trust in the estimation of the trust factor.

Fig. 4.10 shows the mean values and variance for a subsampled map. In Fig. $4.10 \mathrm{~b}$ it is visible that the values of the variance are corresponding to distance to observed locations in CLiFF-map.

\footnotetext{
${ }^{1}$ This dataset covers human motion patterns in the Informatics Forum, the main building of the School of Informatics at the University of Edinburgh. Detailed description of the dataset is page 82.
} 


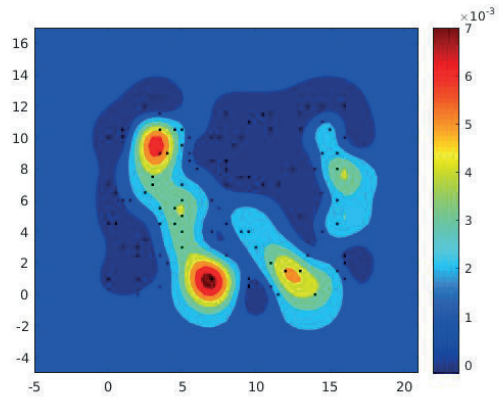

(a) Mean value of GP

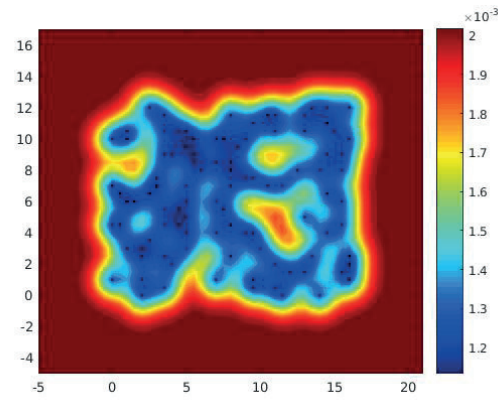

(b) Variance of GP.

Figure 4.10: Visualisation of Gaussian Process representing confidence factor based on sub-sampled set of observations. The black dots mark locations of SWGMM on the map.

This method of computation of trust factor has two major advantages over the method using weighted mean. It combines motion ratio, observation ratio and distance to observed locations, where each of them has an impact on the trust of the estimate. Furthermore, it also provides confidence information regarding the estimated trust values.

\subsection{Evaluation Methodology}

In the following sections I will evaluate two aspects of CLiFF-map:

Mapping - is a process of building a set of SWGMMs from observations.

Densification - is a process of building a set of SWGMMs using imputed data in unobserved locations.

For evaluation of mapping and densification I have used four methods:

Model Selection Criterion - In order to compare the quality of maps obtained with different initialisation methods a Bayesian Information Criterion (BIC) is used. BIC is a penalised log-likelihood which tries to find the best fitting model with the lowest number of degrees of freedom.

Divergence Estimation - In order to provide the quality of the map in absolute values, a divergence estimator proposed by Wang et al. [74] was used. The proposed estimator computes the differences between the estimated model and the underlying data.

$k$-fold cross validation - To evaluate the predictive capabilities of the model $\mathrm{k}-\mathrm{CV}$. 
Stability maps - The methods listed above relies on the access to ground truth. In order to provide insights on map quality in lack of access to such stability maps, were used. Stability maps compare the dense map of the flow for this same location, but for different time windows.

\section{Bayesian Information Criterion}

To measure the relative quality of the fit between different clustering methods BIC was used. BIC can be interpreted as penalised log-likelihood, where the penalty should prevent from preferring models with redundant clusters. The formal definition of BIC is:

$$
\mathrm{BIC}=\ln (\mathrm{n}) \mathrm{k}-2 \ln (\hat{\mathrm{L}}) .
$$

where:

- $\hat{\mathrm{L}}$ - the maximised value of the likelihood function of the model i.e. $\hat{\mathrm{L}}=$ $p(x \mid \hat{\theta}, M)$, where $\hat{\theta}$ are the parameter values that maximise the likelihood functions and $x$ is the the observed data

- $\mathrm{n}$ - number of data points

- $k$ - degrees of freedom of the model

\section{Divergence estimator}

To each location in the map, there is associated a true physical process which is generating observations from an unknown PDF denoted as $\xi^{\mathrm{t}}$. It is impossible to access this distribution directly. We can only access a set of observations $\left\{\mathbf{V}_{1}^{o}, \ldots, \mathbf{V}_{n}^{o}\right\}$. One method to estimate the divergence between two ddimensional distributions, while having access only to samples, was proposed by Wang et al. [74]. Wang et al. propose an estimator that employs only the samples coming from the two distributions. The estimator is computed as follows:

$$
\hat{D}_{n, m}\left(\xi^{t} \| \xi\right)=\frac{d}{n} \sum_{i=1}^{n} \log _{2} \frac{v_{k}(i)}{\rho_{k}(i)}+\log _{2} \frac{m}{n-1}
$$

The idea is to compare the distance $\rho_{k}(i)$ between $\mathbf{V}_{i}^{o}$ and its $k-N N$ in $\left\{\mathbf{V}_{j}^{o}\right\}_{j \neq i}$ to the distance $v_{k}(i)$ between $\mathbf{V}_{i}^{o}$ and its $k-N N$ in $\left\{\mathbf{V}_{j}^{s}\right\}$, where $\left\{\mathbf{V}_{j}^{s}\right\}$ denotes virtual observations sampled from the component of the CLiFF-map.

\section{$k$-fold cross validation}

To fully address the task of model validation I have employed $k$-fold cross validation ( $k-C V)$. $k-C V$ allows to accurately estimate how well the model will be able to predict future observations. In my work, I have split the measurements 
for each location into 10 subsets. Nine of them were used for training, and the tenth was used for validation. For each point from the test data set negative log-likelihood was computed as a measure of fit quality.

\section{Stability Map}

A stability map shows which regions contain a stable flow over multiple measurement sessions. The idea is to build a map of flow for each session and then compare them using Kullback-Leibler divergence (KLD). KLD was introduce by Kullback and Leibler in [40].

To evaluate stability I( $k$ ), I compute a summed pairwise symmetric KullbackLeibler divergence (sKLD) between all corresponding maps:

$$
I(k)=\sum_{i, j \in \mathfrak{n}} s \operatorname{KLD}(k)_{i, j}
$$

where, $n$ is number of maps, $k$ is a currently evaluated cell, and $i$ and $j$ are indices of currently evaluated maps.

The KLD is computed in a discretised state space $\Gamma_{\Theta, \mathrm{P}}$. Each state in $\Gamma_{\Theta, \mathrm{P}}$ is a $\gamma=(\Theta, P)$ tuple of direction and speed where $\Theta \in\left\{\Theta_{\min }, \Theta_{\min }+\Delta \Theta, \ldots, \Theta_{\max }\right\}$ and $\mathrm{P} \in\left\{\mathrm{P}_{\min }, \mathrm{P}_{\min }+\Delta \mathrm{P}, \ldots, \mathrm{P}_{\max }\right\}$. A discretised PDF for each distribution for each location is denoted as $\boldsymbol{x}=\left(x_{\gamma} \mid \gamma \in \Gamma\right)$. Therefore, we can define KLD for kth element in the map as

$$
\operatorname{KLD}\left(x^{i}(k) \| x^{j}(k)\right)=\sum_{\gamma \in \Gamma} x_{\gamma}^{i}(k) \log \frac{x_{\gamma}^{i}(k)}{x_{\gamma}^{j}(k)} .
$$

The sKLD for each cell $(k)$ is computed as

$$
\operatorname{sKLD}(k)_{i, j}=\operatorname{KLD}\left(\boldsymbol{x}^{i}(k) \| \boldsymbol{x}^{j}(k)\right)+\operatorname{KLD}\left(\boldsymbol{x}^{j}(k) \| \boldsymbol{x}^{i}(k)\right)
$$

\subsection{Evaluation}

The goal of this section is to present the CLiFF-maps for different data-sets and to evaluate the quality of maps built from dense and sparse data.

\subsubsection{CLiFF-map's Toy Examples}

The primary feature of CLiFF-map is to grasp the multimodal characteristics of flows. Fig. 4.11 demonstrates key characteristics of the CLiFF-map representation with two toy examples. 


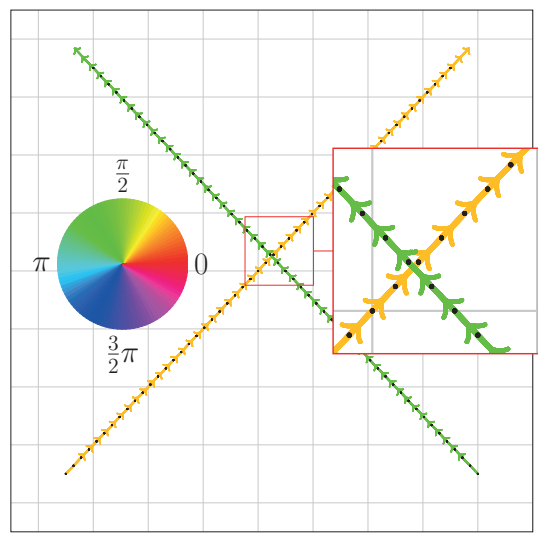

(a) Input: two tracks with the same velocity but different orientation.

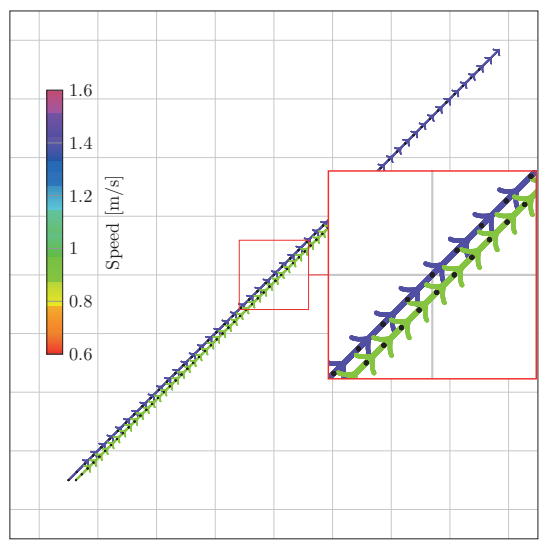

(c) Input: two tracks with different velocity along the same line.

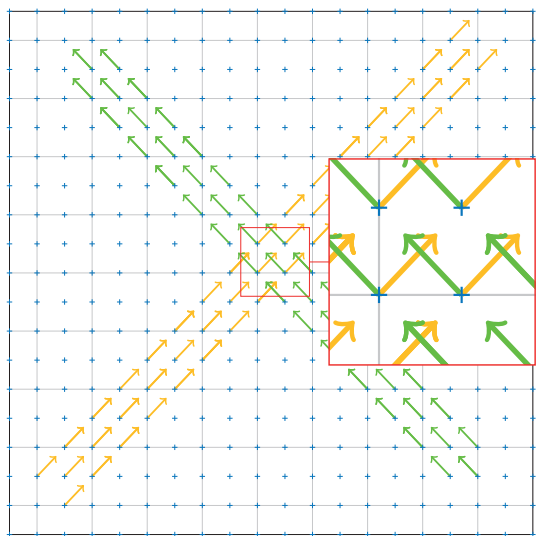

(b) CLiFF-map for 4.11a, with modes for different directions.

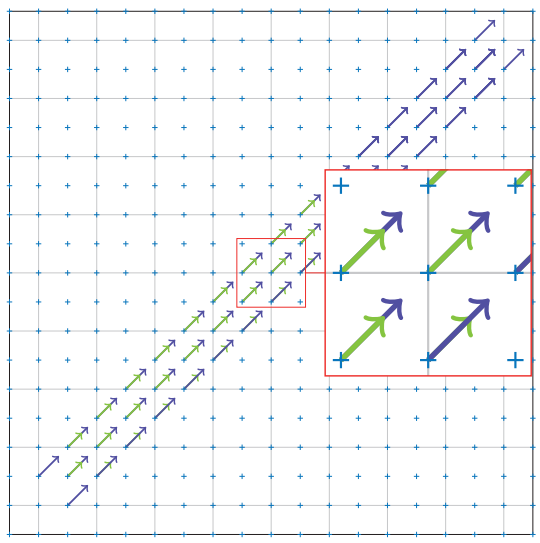

(d) CLiFF-map for $4.11 \mathrm{c}$, with modes for different speeds.

Figure 4.11: A toy example demonstrating how CLiFF-map addresses the problem of multiple modes.

\section{Separation in Orientation}

Fig. 4.11a shows two tracks, black dots represent the measurements locations and arrows represent the measured velocities. (Each track was built using over 1000 simulated measurements and the arrows are just representing general direction of motion.) To build the distributions shown in Fig. 4.11b I have discretised the data in Fig. 4.11a as described in the Sec. 4.3.1. 
We can see that the CLiFF-map preserves the correct orientation of the flows and the velocities. Fig. $4.11 \mathrm{~b}$ shows a zoomed image of the area of the intersection. It is visible that CLiFF-map preserved the information about two directions of motions even for the part where the flows intersect. However, because of the resolution of the map, we can observe an artefact. Namely, the track becomes wider than the original one.

\section{Separation in Speed}

The tracks presented in Fig. 4.11c were generated in the same way as the tracks in Fig. 4.11a. However, this example aims to visualise the capability of a CLiFFmap to preserve the information about differences in speed. Fig. $4.11 \mathrm{~d}$ shows that the CLiFF-map correctly models the two modes coming from different speeds at the same location. In this example, we also observe similar artefacts as in Fig. 4.11 b, caused by the map discretisation.

\subsubsection{Evaluation of Mapping}

The primary focus of this section is on the dependency between the different initialisation methods for EM and the quality of the resulting map. The evaluation is performed with two real-world data sets:

- Edinburgh Pedestrian Dataset (described next),

- Drone Wind Dataset (described on page 89).

\section{Edinburgh Pedestrian Data}

Dataset Description This data set was collected in the School of Informatics at the University of Edinburgh [45]. Between 24th of August 2009 and 1st of August 2010 more than 95998 trajectories of people walking through the Informatics Forum were observed. The camera is fixed overhead (although it might drift and vibrate a little over time) approximately $23 \mathrm{~m}$ above the floor. The images are $640 \times 480$, where each pixel (horizontally and vertically) corresponds to $24.7 \mathrm{~mm}$ on the ground, and the capture rate is about nine frames per second. Fig. 4.12 shows an example of registered detections (the colour coding corresponds to the direction of motion). The velocity measurements were estimated based on translations between the consecutive observations. Based on collected measurements s map representing a flow was built (see Fig. 4.12). Such maps were used for mapping evaluation.

Resulting Map Overview Fig. 4.12a shows the velocity measurements extracted from the data set, the colour corresponds to the direction of the motion. These measurements correspond to paths followed by the pedestrians. The dominant 


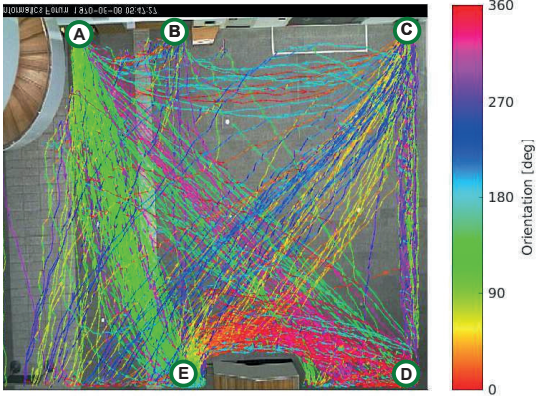

(a) Tracks extracted from the raw tracking data, used as input to CLiFF-map.

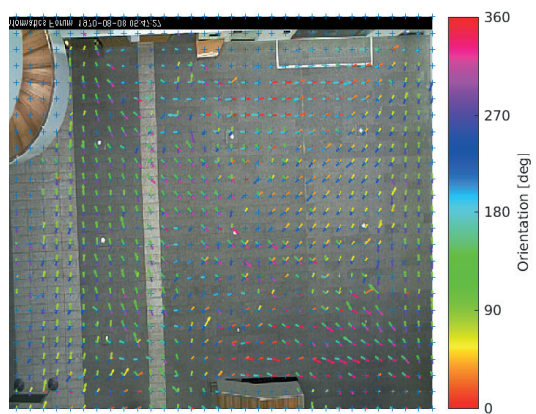

(c) Map including the learned motion directions whose mixing factor is higher than 0.1 . The distance between nodes is $0.5 \mathrm{~m}$.

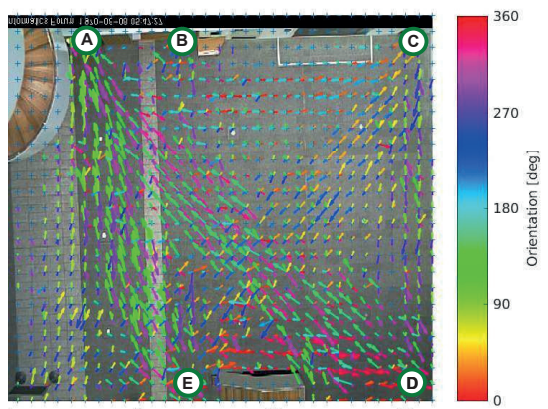

(b) Map including all the learned motion directions. The distance between nodes is $0.5 \mathrm{~m}$.

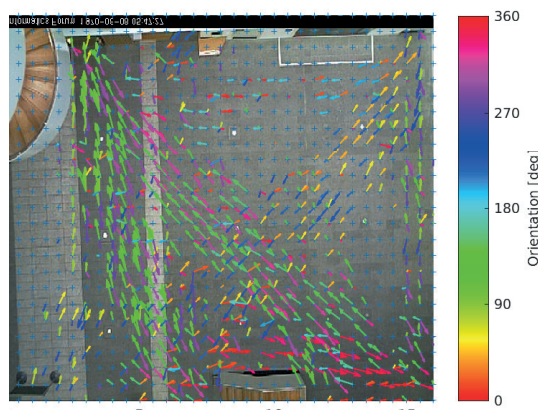

(d) Map including the learned motion directions whose mixing factor is lower than 0.1 . The distance between nodes is $0.5 \mathrm{~m}$.

Figure 4.12: Example of CLiFF-map for Edinburgh pedestrian dataset.

paths are A-E, A-D, B-C, E-D, E-C, C-D. If we compare the learned motion directions in Fig. 4.12b we can see that aforementioned patterns are still visible.

In Fig. $4.12 \mathrm{~b}$ we can see a high variance in the mapped velocities. It looks like people tend to run between points $\mathrm{A}$ and $\mathrm{E}$ and move rather slowly between the points $\mathrm{D}$ and $\mathrm{C}$. This impression comes from the fact that we are not visualising the mixing factors of the modes in the SWGMMs. However, if we split the modes based on the mixing factor, we can see an interesting phenomenon.

In Fig 4.12c we can see the modes whose mixing factor is higher than 0.1; that is, the modes that contribute most to the SWGMM. There is much less discrepancy in arrow length; therefore the speed in each direction looks homogeneous. 
In Fig $4.12 \mathrm{~d}$ we can see the modes with mixing factor lower than 0.1. These two figures show that by using this simple filtering approach, we can extract dominant motion patterns. These results also show that CLiFF-map can learn and represent rare events in an appropriate way.

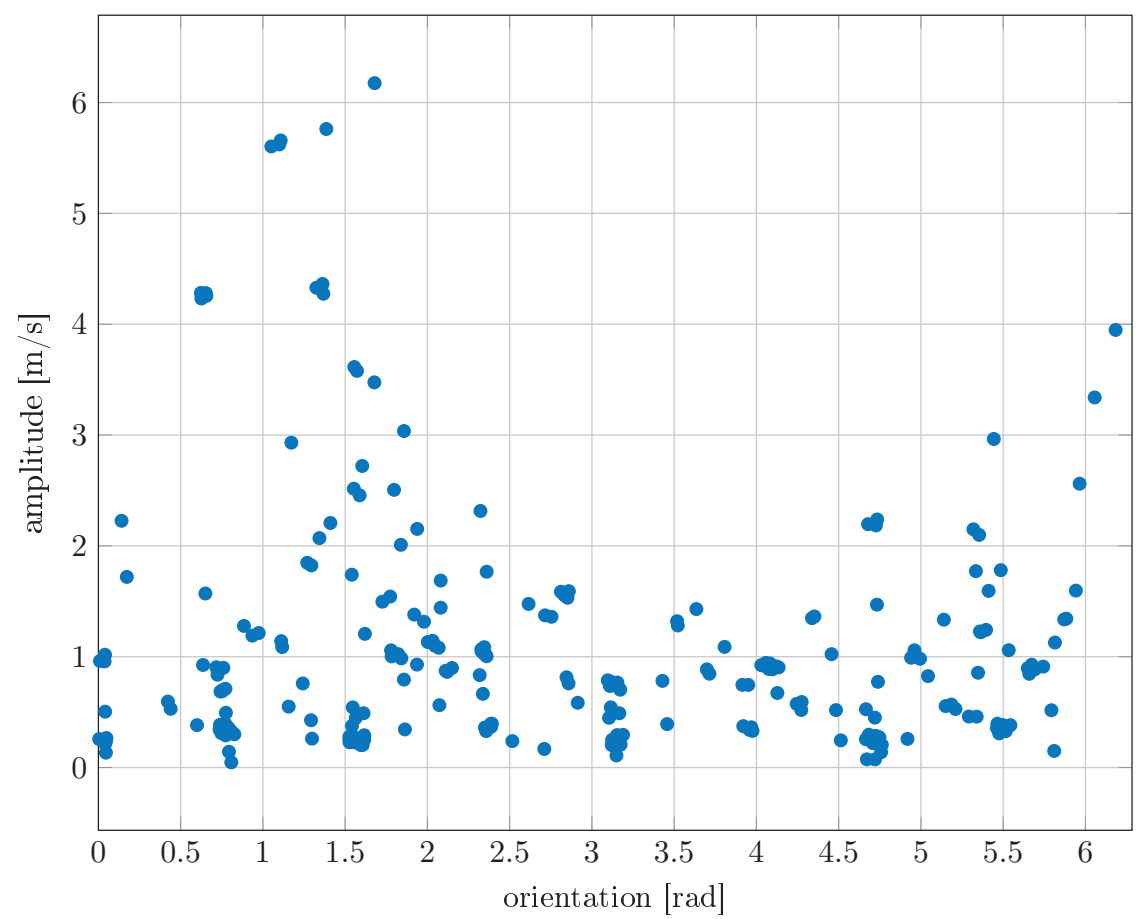

Figure 4.13: Visualisation of velocity measurements for a location in the map shown in Fig. 4.12b

Qualitative Example Let us have a closer look at a single SWGMM and analyse the way the parameters of it area are computed.

In Sec. 4.3.3 I discussed three clustering methods (MS, k-means, OPTICS). I have also pointed out that we can either neglect the fact that the velocity is a heterogeneous quantity (then the methods are called isotropic) or include this information in the clustering process (then the methods are called nonisotropic).

In Fig. 4.14 presents the results of the clustering algorithms. The results vary a lot between the methods. Fig. 4.14a and Fig. 4.14b shows that depending on the distance metrics we obtain two or three clusters. This phenomenon is caused by the fact that for the present case, the non-isotropic kernel penalises 


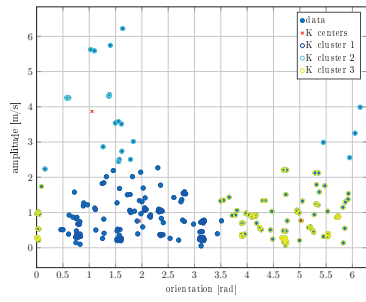

(a) Isotropic k-means.

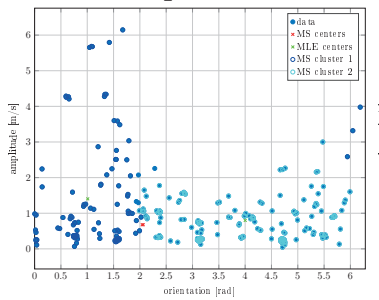

(d) Non-isotropic MS.

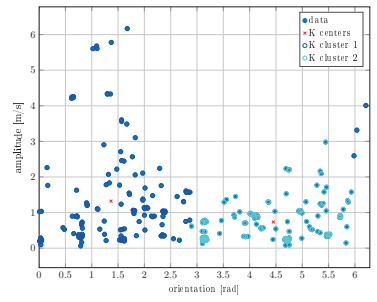

(b) Non-isotropic k-means.

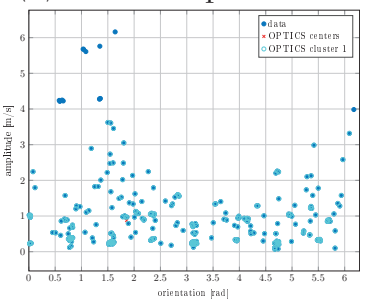

(e) Isotropic OPTICS.

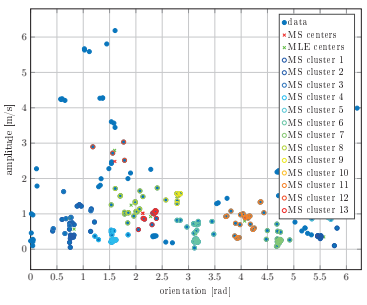

(c) Isotropic MS.

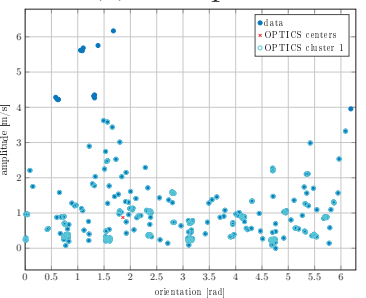

(f) Non-isotropic OPTICS.

Figure 4.14: Examples of initialisation with different clustering methods. Depending on the clustering method different number of clusters is obtained.

disparity in speed less than in velocity. In case of MS we can observe a similar behaviour. An isotropic kernel produces substantially more classes than a nonisotopic kernel. Finally, we can see that clustering with OPTICS results with very few clusters (usually one). This phenomenon is caused by the fact that in this data set, measurements are densely distributed, and there are always some points to connect. Furthermore, only the "remote" points on the very top of the plot are treated as outliers.

After obtaining the initial set of clusters (using any of the methods above) the SWGMM is fitted with EM. In Fig. 4.15 we can observe results of EM for each initialisation type. In all the plots we can see that the modes are in the lower part of the plot, which corresponds to the fact that majority of the objects moved slower than $2 \mathrm{~m} / \mathrm{s}$.

The first interesting observation is that the final position of the modes does not correspond to the position of the initial clusters. Let us compare Fig. 4.14a and Fig. 4.15a. In Fig. 4.14a the yellow and dark-blue cluster are dividing the data points in the lower part of the plot into two groups, which have opposite orientation. The cyan cluster, group points with higher speeds. In contrast in Fig. 4.15a the data points in the lower part of the plot are contributing to one single elongated mode, and the data points in the upper part of the plot are split into two clusters depending on the orientation. A similar shift can be observed in Fig. 4.14b and Fig. 4.15b, and Fig. 4.14d and Fig. 4.15d. In these cases, 


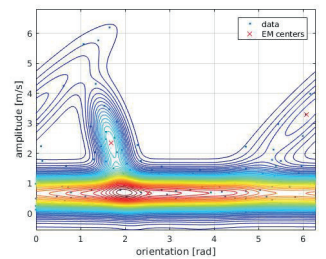

(a) Isotropic k-means.

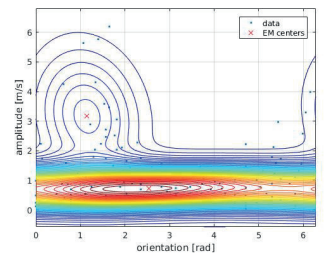

(d) Non-isotropic MS.

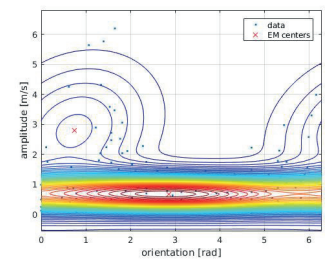

(b) Non-isotropic k-means.

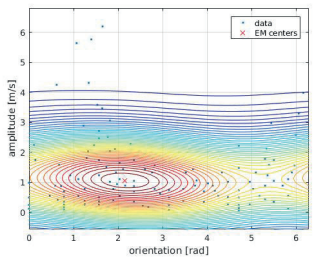

(e) Isotropic OPTICS.

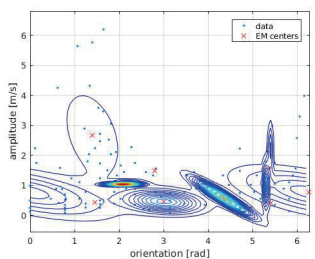

(c) Isotropic MS.

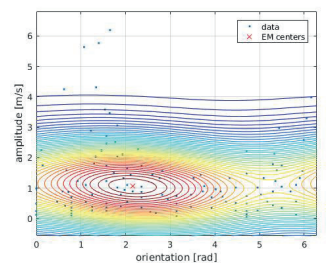

(f) Non-isotropic OPTICS.

Figure 4.15: SWGMM obtained with different initialisation methods.

we can see, that the clustering algorithm divided the data points according to orientation but in the final SWGMM we can observe two modes. The bottom one is covering data-points corresponding to slower objects, and the top one covers fast moving objects. The results for initialisation with OPTICS are the least interesting, one single cluster results in one single distribution with mean pointing near the dominant direction.

In contrast to the results discussed so far, the SWGMM obtained for initialisation with isotropic MS not only differentiate the modes because of the speed but also depending on the orientation.

Quantitative evaluation The quantitative evaluation was performed with the first 9 days of Edinburgh dataset.

The goal of quantitative evaluation is to assess the quality of the representation and measure the impact of different clustering methods on the representation quality. The quality of the map was evaluated using three different metrics Wang divergence, BIC and k-CV (see Sec. 4.5). Furthermore, I also evaluate the number of clusters generates by each initialisation method. This information allows estimating the memory consumption for each initialisation method. Finally in order to provide a better understanding of the quality of the model I provide a baseline, which is a divergence for a histogram. The sizes of the bins for the histogram were computed with Scot's rule [68]. The results of the evaluation are presented in Tab. 4.1 and Fig. 4.16. 


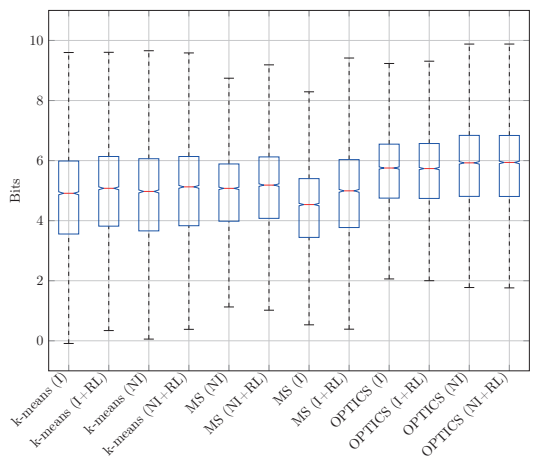

(a) Wang divergence

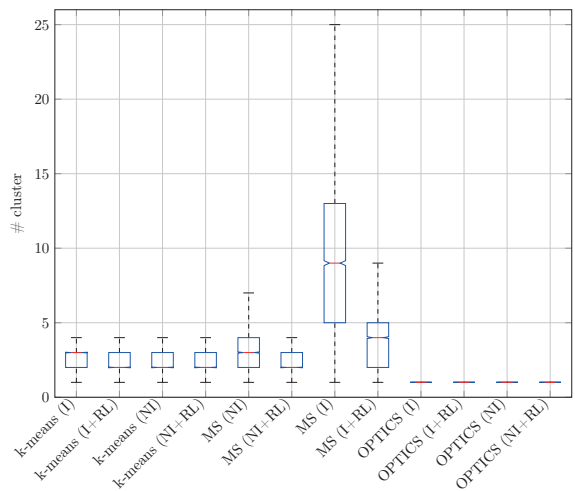

(c) Number of clusters

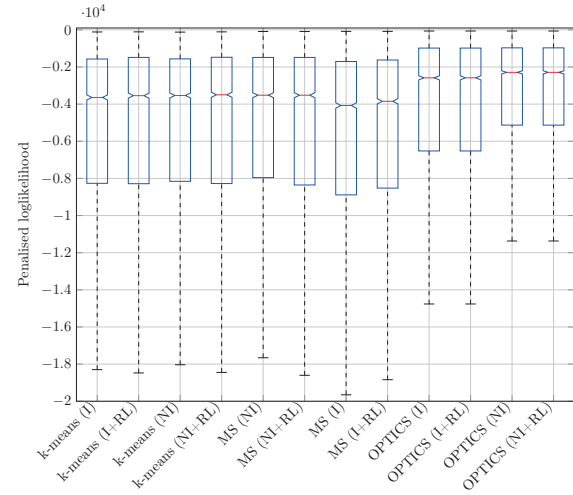

(b) BIC score

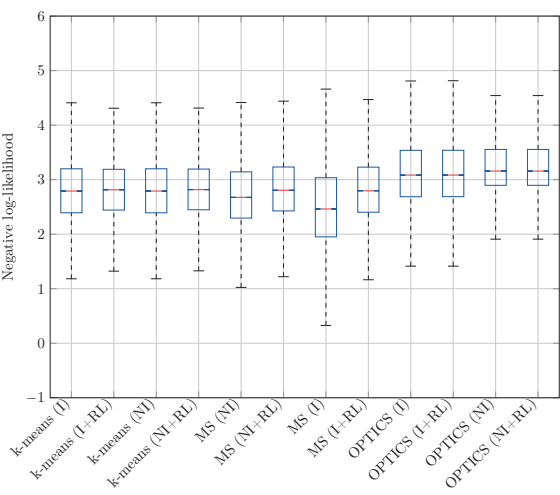

(d) Results for k-CV

Figure 4.16: Evaluation results for Edinburgh pedestrian data based on the first 9 days of the data set. (I) - Isotropic, (NI) - Non-isotropic, (RL) - ridge-line analysis.

In Tab. 4.1 in column 2 there are listed average values of divergence. Furthermore, in brackets, there is provided ordering of the methods according to the divergence. If we compare the score of divergence with the average number of clusters (Tab. 4.1 column 3) we can observe following dependency, the more clusters, the smaller divergence. This result is supporting an intuition that increasing number of modes should lower the divergence between the data and the model.

In contrast to divergence, cross-validation does not prefer too few or too many clusters. The lowest negative log-likelihood corresponding to best predictive capabilities of the model is obtained with a model with a medium number of clusters. Both extremes, too few and too many clusters correspond to a 


\begin{tabular}{|c|c|c|c|c|}
\hline Method & Div. [bit] & BIC & \# clust & $\mathrm{k}-\mathrm{CV}$ \\
\hline 1 & 2 & 3 & 4 & 5 \\
\hline k-means (I) & $\begin{array}{l}4.6638 \\
(2)\end{array}$ & -7122.0 & 3.9045 & 3.3669 \\
\hline k-means $(\mathrm{I}+\mathrm{RL})$ & $\begin{array}{l}4.9135 \\
(6)\end{array}$ & -7144.9 & 2.55 & 3.2564 \\
\hline k-means (NI) & $\begin{array}{l}4.7504 \\
(3)\end{array}$ & -7068.2 & 3.3957 & 3.3642 \\
\hline k-means (NI+RL) & $\begin{array}{l}4.9318 \\
(7)\end{array}$ & -7148.9 & 2.4382 & 3.2217 \\
\hline Mean Shift (NI) & $\begin{array}{l}4.8555 \\
(4)\end{array}$ & -6224.1 & 3.1084 & 2.96 \\
\hline Mean Shift (NI+RL) & $\begin{array}{l}5.0310 \\
(8) \\
\end{array}$ & -6927.0 & 2.3266 & 3.1185 \\
\hline Mean Shift (I) & $\begin{array}{l}4.4003 \\
(1)\end{array}$ & -6976.5 & 10.015 & 3.5887 \\
\hline Mean Shift (I+RL) & $\begin{array}{l}4.8835 \\
(5) \\
\end{array}$ & -6860.2 & 4.1516 & 3.9232 \\
\hline OPTICS (I) & $\begin{array}{l}5.5957 \\
(10)\end{array}$ & -5517.6 & 1.1779 & 3.1805 \\
\hline OPTICS (I+RL) & $\begin{array}{l}5.5883 \\
(9)\end{array}$ & -5516.8 & 1.1752 & 3.1848 \\
\hline OPTICS (NI) & $\begin{array}{l}5.7363 \\
(12)\end{array}$ & -4089.5 & 1 & 3.2661 \\
\hline OPTICS (NI+RL) & $\begin{array}{l}5.7327 \\
(11)\end{array}$ & -4089.5 & 1 & 3.2661 \\
\hline Histogram & 5.4313 & & - & \\
\hline
\end{tabular}

Table 4.1: Numerical results for quality of mapping estimation for Edinburgh pedestrian data set. (I) - Isotropic, (NI) - Non-isotropic, (RL) - ridgeline analysis. The values are mean scores. The numbers in brackets in the second column represent the ordering of the divergence values from the best (smallest divergence) to the worst (biggest divergence).

lower performance. Considering the qualitative results presented in the previous section, we can say that small number of clusters implies the clusters to be rather big, while the high number of clusters corresponds to smaller clusters. That leads to a conclusion that a high number of clusters lead to over-fitting and the model loses its predictive capabilities. On the other hand, just a few big clusters also lower the predictive capabilities, by under-fitting.

A comparison between the results in Tab. 4.1 and Fig. 4.16d shows a discrepancy between the mean and median of MS (I). This discrepancy is caused 
by the fact that scores obtained with k-CV follow a skewed distribution. MS (I) is suffering from outliers with very bad k-CV scores in contrast to the scores for MS (NI). Therefore, while building CLiFF-map it is necessary to make a design choice if we aim for a model which better behaves in extreme cases or are we looking for a better generative model for the major number of modelled locations.

Furthermore, BIC behaves indicates that the best are the models with only one distribution. The primary reason for that is BIC prefers models, which are more biased over models with high variance. It seems like for this type of data it is not the best criterion for model selection.

Finally, in order to provide a baseline comparison histograms were built for each of the locations in the maps. The sizes of the bins in the histogram were computed using Scott's rule [68]. Scott's rule is optimal for random samples of normally distributed data, in the sense that it minimises the integrated mean squared error of the density estimate. The histogram was outperformed (according to divergence) by all of the models that on average used more than one distribution (OPTICS in all four cases resulted almost always in only one cluster). That brings a conclusion that it is necessary to reach for multimodal models in order to not only efficiently but also accurately represent the flow of people.

\section{Drone Wind Data}

Data description In this section, I analyse the quality of maps built for wind data collected with a drone as described in work of Neumann et al. [54]. The data set was collected by a drone which has visited a number of predefined locations in an outdoor environment and collected wind measurements in each of the locations. In Fig. 4.17 we can see a visualisation of one of the data sets.

Quantitative evaluation The maps built with the drone dataset were evaluated in the same way as the previously discussed Edinburgh pedestrian dataset. The numeric results of evaluation are presented in Tab. 4.2 and Fig. 4.2.

The ordering of the initialisation methods according to the divergence score for the Edinburgh pedestrian dataset and drone dataset is almost identical (the only difference is ordering of OPTICS methods). This lead to a conclusion that there is an ordering of initialisation methods, which correspond to a quality of the final model (measured as divergence). Furthermore, the value of divergence also corresponds to the number of clusters. These two facts lead to a conclusion that, there is a connection between the initialisation methods, divergence and the number of modes in the model. This allows the user to chose such an initialisation method which produce a model appropriate to the user's needs (more clusters but smaller divergence or fewer cluster but bigger divergence). This choice can be intuitively understood as a choice between a precise model and more memory efficient model. 


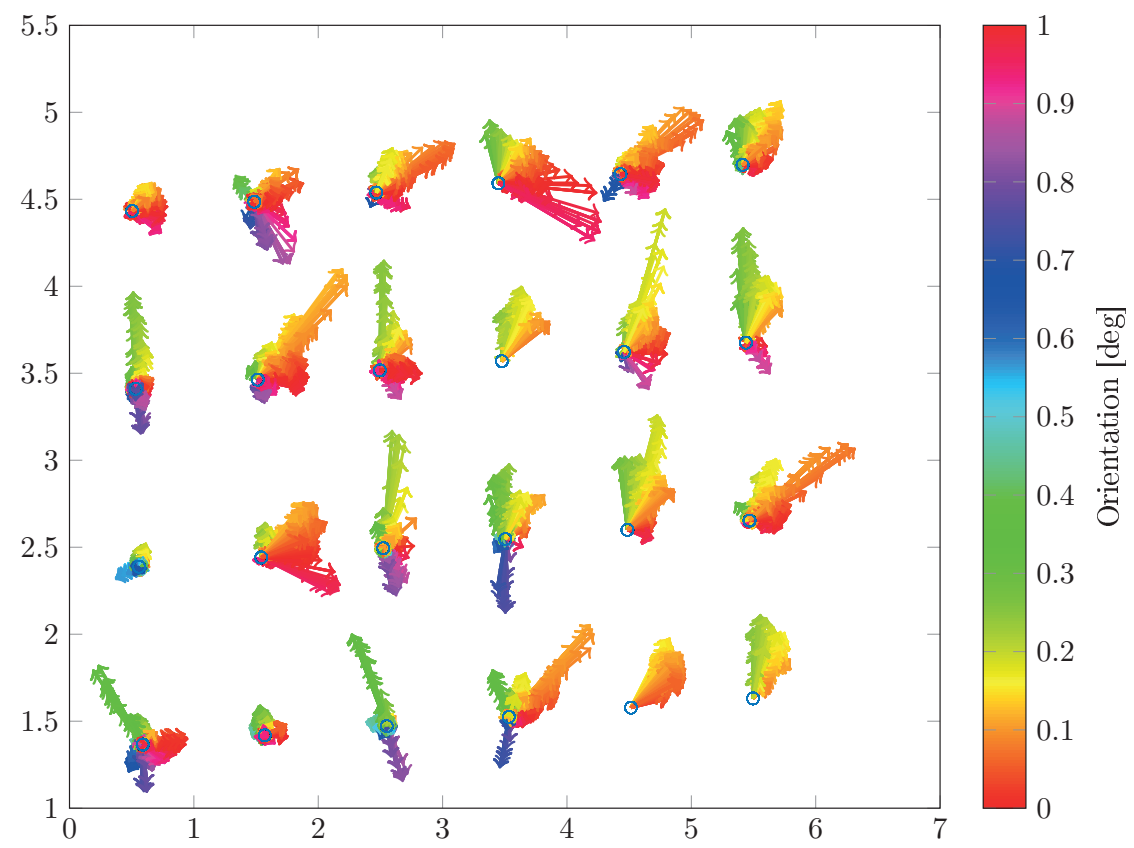

Figure 4.17: Measurement locations and wind measurements for a single run with a drone data. The measurements were collected for aprox. $20 \mathrm{~s}$ per location.

In contrast to results for Edinburgh pedestrian dataset in drone dataset, the good predictive capabilities of the models correspond to models with a higher number of modes. This is caused by the fact, that in the wind data set is not as smooth as the pedestrian dataset, and contains multiple small clusters of data points corresponding to wind velocity changes in turbulent flow (compare Fig. 4.15 with Fig 4.18).

Furthermore, the results for BIC are different between the pedestrian and the drone data set. For pedestrian dataset, BIC preferred models consist of fewer clusters in the drone dataset the models with more clusters are preferred. This comes from the fact that the wind data set is more turbulent and there are separate clusters for changes in wind, while in the pedestrian flow it is more difficult to extract such clusters.

Finally, also for drone dataset, in the majority of the cases, the divergence between the data and the obtained model is smaller than for the histogram. Similarly to Edinburgh pedestrian dataset, also for drone dataset, a low number of clusters corresponds to the low quality of the model. This observation further 


\begin{tabular}{|c|c|c|c|c|}
\hline Method & $\begin{array}{l}\text { Div. } \\
\text { [bit] }\end{array}$ & $\mathrm{BIC}$ & \# clust & $\mathrm{k}-\mathrm{CV}$ \\
\hline 1 & 2 & 3 & & \\
\hline k-means (I) & $\begin{array}{l}2.8699 \\
(2)\end{array}$ & -12288 & 11.273 & 1.5256 \\
\hline k-means (I+RL) & $\begin{array}{l}3.376 \\
(6)\end{array}$ & -12423 & 4.3333 & 1.7156 \\
\hline k-means (NI) & $\begin{array}{l}3.0112 \\
(3)\end{array}$ & -12162 & 9.2933 & 1.5256 \\
\hline k-means (NI+RL) & $\begin{array}{l}3.4171 \\
(7)\end{array}$ & -12456 & 3.8833 & 1.7257 \\
\hline Mean Shift (NI) & $\begin{array}{l}3.3097 \\
(4)\end{array}$ & -12423 & 3.8067 & 1.5984 \\
\hline Mean Shift (NI+RL) & $\begin{array}{l}3.521 \\
(8)\end{array}$ & -12759 & 2.3867 & 1.7295 \\
\hline Mean Shift (I) & $\frac{2.2968}{(1)}$ & -12497 & 24.317 & 1.3034 \\
\hline Mean Shift (I+RL) & $\begin{array}{l}3.3725 \\
(5)\end{array}$ & -12686 & 5.5367 & 1.7277 \\
\hline OPTICS (I) & $\begin{array}{l}3.8361 \\
(10)\end{array}$ & -12983 & 1.1867 & 1.9113 \\
\hline OPTICS (I+RL) & $\begin{array}{l}3.8273 \\
(9)\end{array}$ & -12980 & 1.1633 & 1.9164 \\
\hline OPTICS (NI) & $\begin{array}{l}3.8782 \\
(11)\end{array}$ & -13033 & 1 & 1.9507 \\
\hline OPTICS (NI+RL) & $\begin{array}{l}3.8828 \\
(12)\end{array}$ & -13033 & 1 & 1.9507 \\
\hline "Histogram & 3.8563 & - & - & - \\
\hline
\end{tabular}

Table 4.2: Numerical results for quality of mapping estimation for drone data set. (I) - Isotropic, (NI) - Non-isotropic, (RL) - ridgeline analysis. The values are mean scores. The numbers in brackets in the second column represent the ordering of the divergence values from the best (smallest divergence) to the worst (biggest divergence).

reinforces the conclusion that multimodal models are better suited to model a flow related real-world phenomena.

\section{Conclusions}

The aim of this section was on the one hand to present the capabilities of CLiFF-map for different datasets and on the other hand to evaluate the quality of the obtained map. 


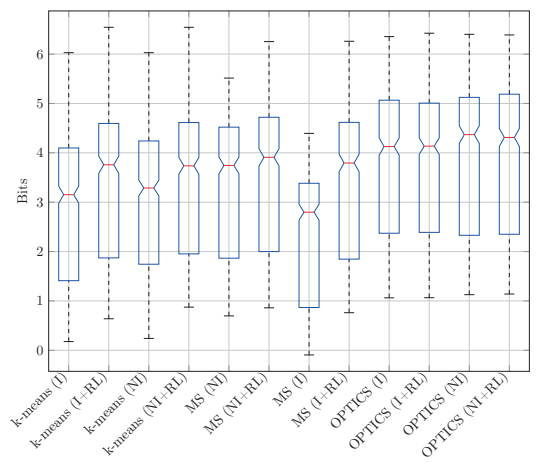

(a) Wang divergence.

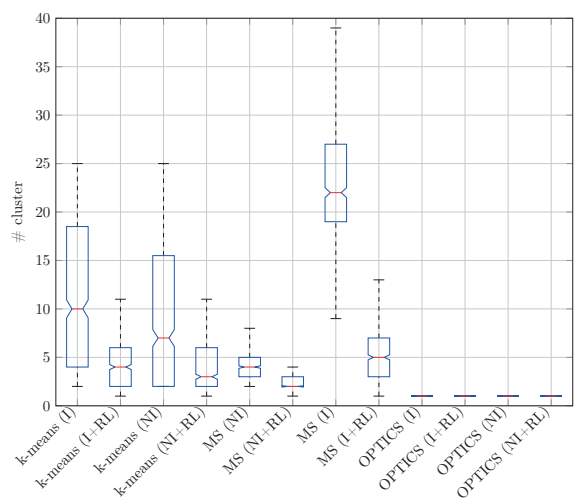

(c) Number of clusters.

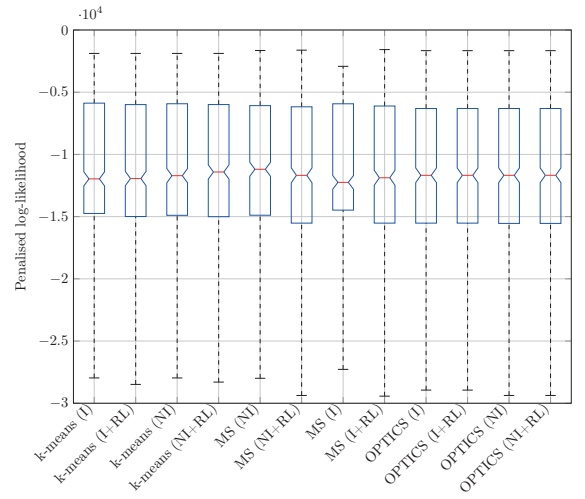

(b) BIC score.

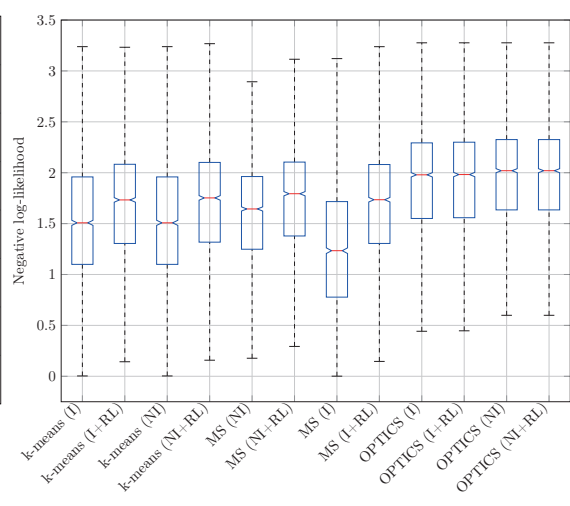

(d) Results for k-CV.

Figure 4.18: Evaluation results for Drone data. (I) - Isotropic, (NI) - Nonisotropic, $(\mathrm{RL})$ - ridgeline analysis.

In Sec. 4.6.1 it was shown, that CLiFF-map is able to preserve the information about multimodal motion patterns both in orientation and velocity.

Furthermore, I have discussed the impact of different initialisation methods on the quality of the resulting maps. First, it is important to emphasise there is a clear dependency between the initialisation method and the average number of modes describing flow in the location. Moreover, there is a clear relation between the number of clusters, type of initialisation and the quality of the model (measured as divergence). From the application point of view, this information allows picking a type of initialisation according to the desired level of detail in the model. 


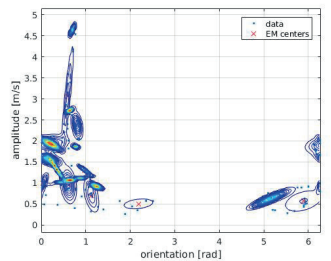

(a) Isotropic MS.

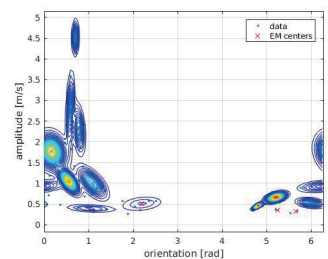

(b) Isotropic k-means.

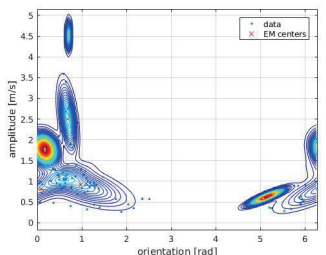

(c) Non-isotropic MS.

Figure 4.19: SWGMM obtained with different initialisation for a location in a drone datset.

The conducted experiments also show that removing clusters with ridgeline analysis is in most of the cases is decreasing the quality of the obtained model according to all three measures. The only exception is OPTICS, where the quality of resulting map is not changed.

The comparison between the Tab. 4.1 and Tab. 4.2 shows that in all cases independently from the initialisation method the quality of mapping is better for the drone data set. This also applies to the base-line method using the histogram. A closer look at the data shows that these datasets have different levels of dispersion. Intuitively dispersion is a measure of how much the data is stretched or squeezed. I have computed average dispersion for both data sets along each dimension separately. The dispersion of angular values was computed according to the definition provided by Mardia and Jupp in [47]. Using notation introduced in Eq. (4.15) and Eq. (4.14) the dispersion is defined as:

$$
\overline{\mathrm{R}}=(\overline{\mathrm{C}}+\overline{\mathrm{S}})^{1 / 2} \text {. }
$$

The dispersion of the angular data is bounded to the following interval:

$$
0 \leqslant \bar{R} \leqslant 1
$$

It is important to emphasise that tightly clustered data will be almost 1 while for a widely spread data set dispersion will be close to 0 . While for the speed data as a measure of dispersion variance was used.

\begin{tabular}{|l|c|c|}
\cline { 2 - 3 } \multicolumn{1}{c|}{} & Orientation & Speed \\
\hline Drone data & 0.6869 & 0.0512 \\
\hline Pedestrian data & 0.1606 & 0.0223 \\
\hline
\end{tabular}

Table 4.3: Comparison of dispersion for pedestrian and drone data.

In Tab. 4.3 it is shown that the drone data are more concentrated than the pedestrian data. In case of pedestrian data, we can see that on average the 
data is distributed more evenly over the unit cylinder. The difference in the angular dispersion might be one of the reasons why the quality of the model estimation differs so much. Furthermore, a closer look at the pedestrian data shows that the velocity measurements are following an asymmetric distribution. Namely, the highest concentration of observations is around $1 \mathrm{~m} / \mathrm{s}$ speed with a minimum speed of $0 \mathrm{~m} / \mathrm{s}$ and relatively long tail of high speeds. Such shaped distribution is not well modelled with a symmetric normal distribution.

\subsubsection{Evaluation of Densification}

In the previous section I have analysed the quality of mapping with CLiFF-map. Namely, I was evaluating how well the proposed model describes the underlying data. As I have mentioned at the beginning of the Sec. 4.6 I will also evaluate the quality of the densification. The goal of the densification process (see. 4.4) is to estimate the measurements in the unobserved locations and based on them compute the models of patterns of dynamics.

I have evaluated the densification using three data sets: pedestrian dataset, drone dataset and foundry data set. The first two data sets were already discussed in the previous section (see Sec. 4.6.2 and Sec. 4.6.2 respectively) the third one is a data set collected with a robot in a foundry. The details of this data set will be discussed in the following part of the section.

\section{Pedestrian Data}

In order to evaluate the quality of densification based on data imputation, I have sub-sampled the original maps and used such inputs to build the complete map. In Fig. 4.20 we can see an example of a map where $25 \%$ of locations remained in the map followed by two maps densified with MC and NW imputation. While in Fig. 4.21 we can see a result of densification where only $3 \%$ of original observed locations were used.

Fig. 4.20b shows a denisifed map built using MC with a kernel size $0.25 \mathrm{~m}$. The dominant diagonal direction is preserved, and the motionless area on the top-left corner of the map was estimated correctly. Also, the vertical and horizontal motion patterns along the bottom and the left edge of the map remained visible.

Fig. 4.20c shows a desnification of the map with NW, also with kernel size $0.25 \mathrm{~m}$. The quality of the resulting map is much lower. There is a clear line dividing the map into two areas, and the motion patterns are averaged over a big area.

In Fig. 4.21 a more challenging example is presented, where only $3 \%$ of the locations remained and were used for estimation of a dense map. The result of densification with Monte Carlo methods (see Fig. 4.21b) still appear to be qualitatively correct. The motion patterns on the left and in the upper right corner are still visible, but the information about the motion in the lower right 
corner is lost. In Fig. 4.21c, the results of densification with Nadaraya-Watson looks similar to the previous one. We can see a clear-cut into two areas and over smoothing.

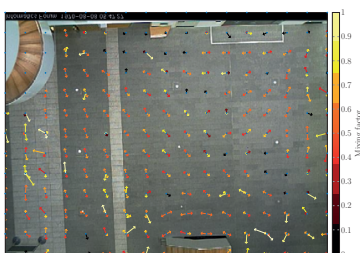

(a) Sub-sampled map used as an input.

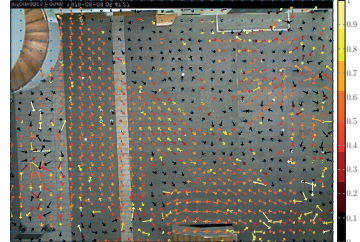

(b) Map densified with MC imputation, kernel size $0.25 \mathrm{~m}$.

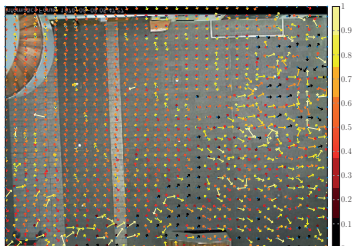

(c) Map densified with NW imputation, kernel size $0.25 \mathrm{~m}$.

Figure 4.20: Densification of a map based on 221 locations ( $1 \mathrm{~m}$ between input locations), shown as blue crosses in (a). (I show only means of modes with mixing factor higher than 0.05.)

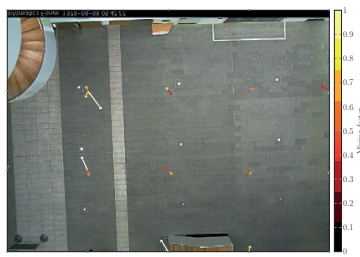

(a) Sub-sampled map used as input.

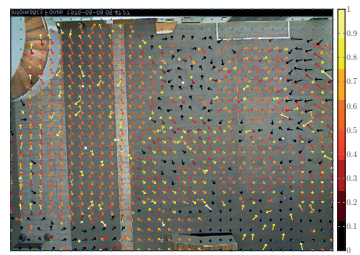

(b) Map interpolated with MC, kernel size $1 \mathrm{~m}$.

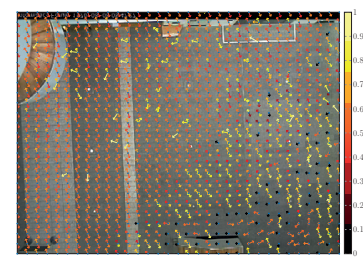

(c) Map interpolated with NW, kernel size $1 \mathrm{~m}$.

Figure 4.21: Densification of a map based on 20 locations ( $4 \mathrm{~m}$ between input locations), shown as blue crosses in (a). (I show only means of modes with mixing factor higher than 0.05.)

To quantitatively measure the quality of the densification, I compute the divergence between the learned SWGMMs and the original data using the estimator of Wang et al. [74].

Fig. 4.22 shows a map of divergence accompanied by the histogram of the computed distances. The distances are concentrated around a value of 5 bits. Fig. 4.23 shows quality of map densification based on Monte Carlo imputation. The distances are concentrated as in Fig. 4.22b, however, the average bit count is higher. There is a similar tendency for map densification with Nadaraya Watson imputation. In Fig. 4.24b, the histogram and divergence map looks similar as in the previous examples.

To properly evaluate the quality of densification, I have measured the divergence for densified maps for nine days, each consist of 825 cells, that is 7425 data points, it took on average 8 seconds to estimate parameters for each location. 

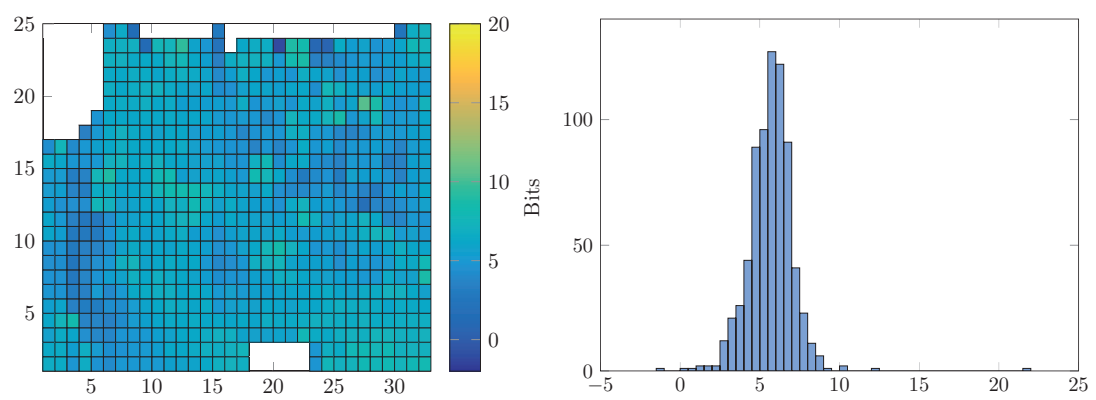

(a) A plot of the distance between the (b) Histogram of divergence values based ground truth measurements and the result on $4.22 \mathrm{a}$. of mapping.

Figure 4.22: Analysis of mapping.
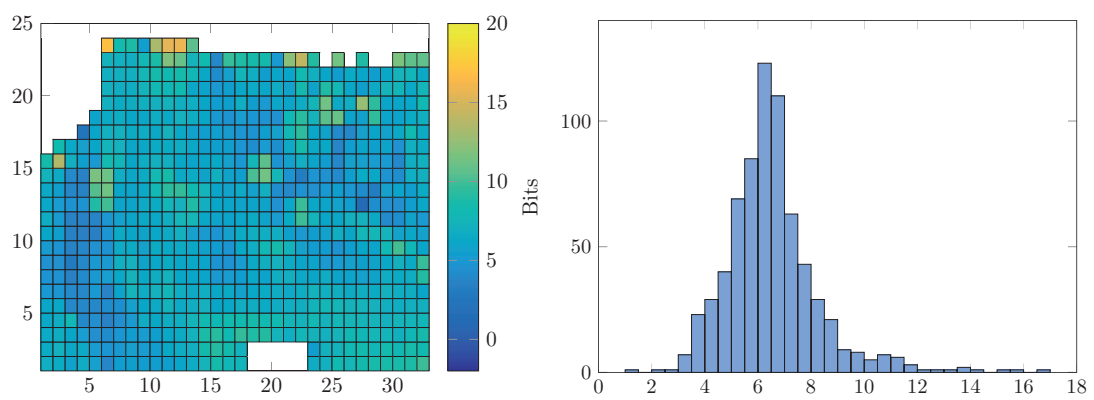

(a) A plot of the distance between the ve- (b) Histogram of divergence values based locity measurements and the result of in- on $4.23 \mathrm{a}$. terpolation.

Figure 4.23: Analysis of densification with Monte Carlo imputation.

In Fig. 4.25 I present how the quality of densification depends on the data imputation method and the size of the kernel. For this experimental setup, the densification quality depends on the amount of input data. However, in both cases, the results are close to the quality of the original map. The MC method shows to be overall better than NW method, besides of the one case in $3 \%$ data set, in which, for a small kernel, the NW method is better.

However, while the quality of densification with MC improves with the growth of the kernel size, the quality of densification with NW method decreases.

Similarly, I have computed a baseline for map densification. To obtain a missing histogram, I have linearly interpolated four nearest histograms. In this 

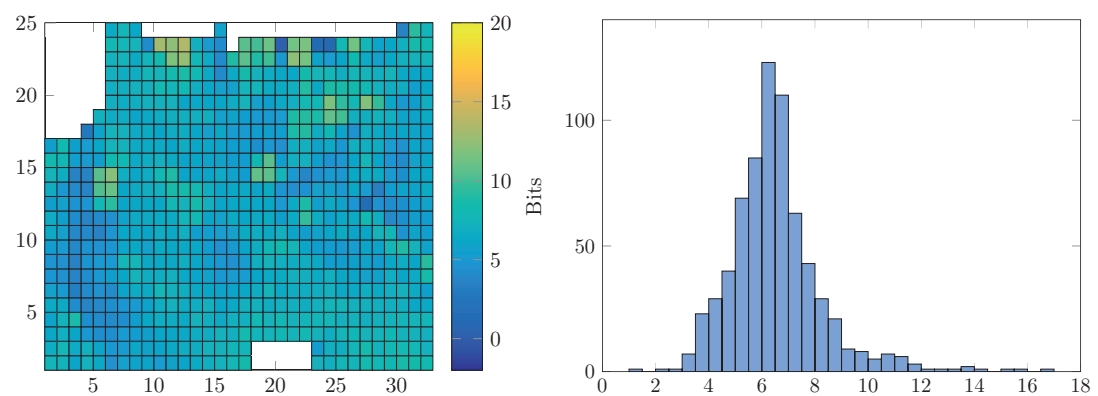

(a) A plot of the distance between the ve- (b) Histogram of divergence values based locity measurements and the result of in- on $4.24 \mathrm{a}$. terpolation.

Figure 4.24: Analysis of densification with Nadaraya Watson imputation.

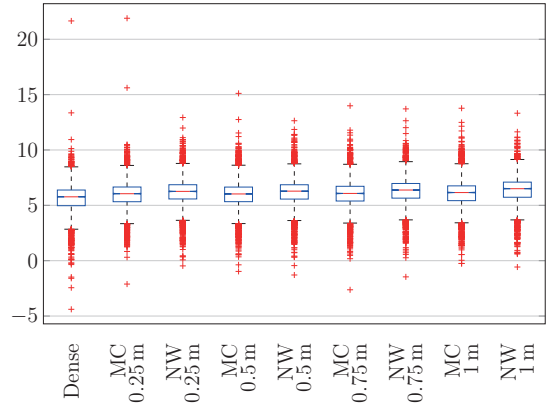

(a) $75 \%$ of initial data

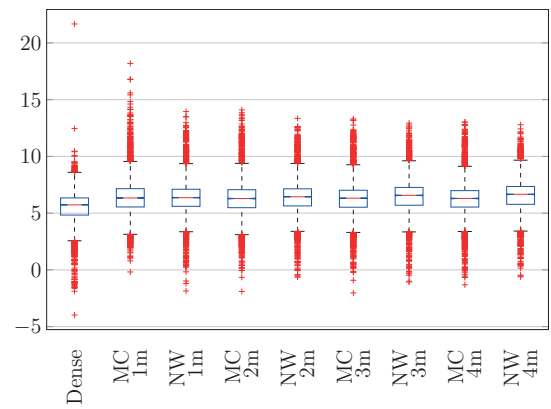

(b) $3 \%$ of initial data.

Figure 4.25: The box-plot of divergence for dense maps

case, we see that the quality of densification depends on the density of input data. For $1 \mathrm{~m}$ resolution both, MC and NW, outperforms interpolation. In $\mathrm{Tab} 4.4$ we can see that for $4 \mathrm{~m}$ resolution the quality of densification with $\mathrm{MC}$ and NW is similar to the result of interpolation.

\section{Drone Data}

In Sec. 4.6.3 it was shown how well CLiFF-map builds dense map from sparse observations for a pedestrian data. In this section, the focus will be on the quality of densification with wind data.

In this case, we do not have access to an arbitrarily dense data set; therefore the strategy of densification evaluation used in Sec. 4.6.3 cannot be used anymore. Instead of sub-sampling a map and estimating the omitted locations a 


\begin{tabular}{c|cc|cc|cc} 
date & \multicolumn{2}{|c|}{ Interp. } & \multicolumn{2}{c|}{ MC } & \multicolumn{2}{c}{$\mathrm{NW}$} \\
& $1 \mathrm{~m}$ & $4 \mathrm{~m}$ & $1 \mathrm{~m}$ & $4 \mathrm{~m}$ & $1 \mathrm{~m}$ & $4 \mathrm{~m}$ \\
\hline 01.09 & 6.1909 & 6.2167 & 6.0787 & 6.5309 & 6.1493 & 6.4698 \\
02.09 & 6.1497 & 6.2430 & 5.7773 & 6.1749 & 5.9931 & 6.1229 \\
04.09 & 6.1339 & 6.1436 & 5.9523 & 6.3966 & 6.2251 & 6.3725 \\
05.09 & 6.2953 & 6.2940 & 5.9955 & 6.3962 & 6.2290 & 6.4081 \\
06.09 & 6.3561 & 6.5055 & 5.9606 & 6.2894 & 6.2130 & 6.3771 \\
10.09 & 5.8692 & 5.9724 & 5.5287 & 6.0253 & 5.7254 & 6.2298 \\
11.09 & 5.5089 & 5.5684 & 5.1614 & 5.6638 & 5.4101 & 5.7552 \\
12.09 & 6.5107 & 6.5902 & 4.3455 & 5.6330 & 5.0089 & 5.7810 \\
13.09 & 5.3982 & 5.5044 & 5.0311 & 5.6712 & 5.5432 & 5.7759 \\
\hline Mean & 6.0459 & 6.1154 & 5.5268 & 6.0868 & 5.8330 & 6.1436
\end{tabular}

Table 4.4: Baseline comparison

Leave One Location Out (LOLO) method will be used. The idea behind LOLO evaluation is to leave one of the locations out and estimate the corresponding distribution based on the remaining ones. The quality metrics is the divergence between the original data set and estimated distribution. In Fig. 4.26 we can see the quality of densification depending on the used method and kernel size.

The magenta plot represents the quality of the densification with MC while the blue one represents the densification with NW. In this plot, we can see that the median divergence of MC densification does not change with the size of the kernel and remain almost unchanged. It is also important to mention that disparity of the error decrease with the increasing size of the kernel. In other words, the bigger the kernel, the better densification in the worst case scenario.

On the other hand, we can see a clear increase in the divergence for densification with NW. Not only, the median increase but also the dispersion increase. In other words, the bigger the kernel the worst densification for the worst case scenario.

We can observe a similar discrepancy (however not as amplified) in the quality of the densification also for the pedestrian data. The conclusion here is that NW method attempt to smooth-out the data while MC attempts to preserve the turbulent character of the modelled flow.

\section{Foundry Data}

The goal of this subsection is to present the results of CLiFF-map densification for a more complex dataset. The dataset used in this subsection was collected by Bennetts et al. [8]. The wind data was collected in a foundry, using a mobile platform. The size of the environment is $40 \mathrm{~m} \times 70 \mathrm{~m}$. The robot was deployed for several data collection tours. In each measurement tour, the robot stopped at different waypoints and collected measurements for $120 \mathrm{~s}$. Using data from 


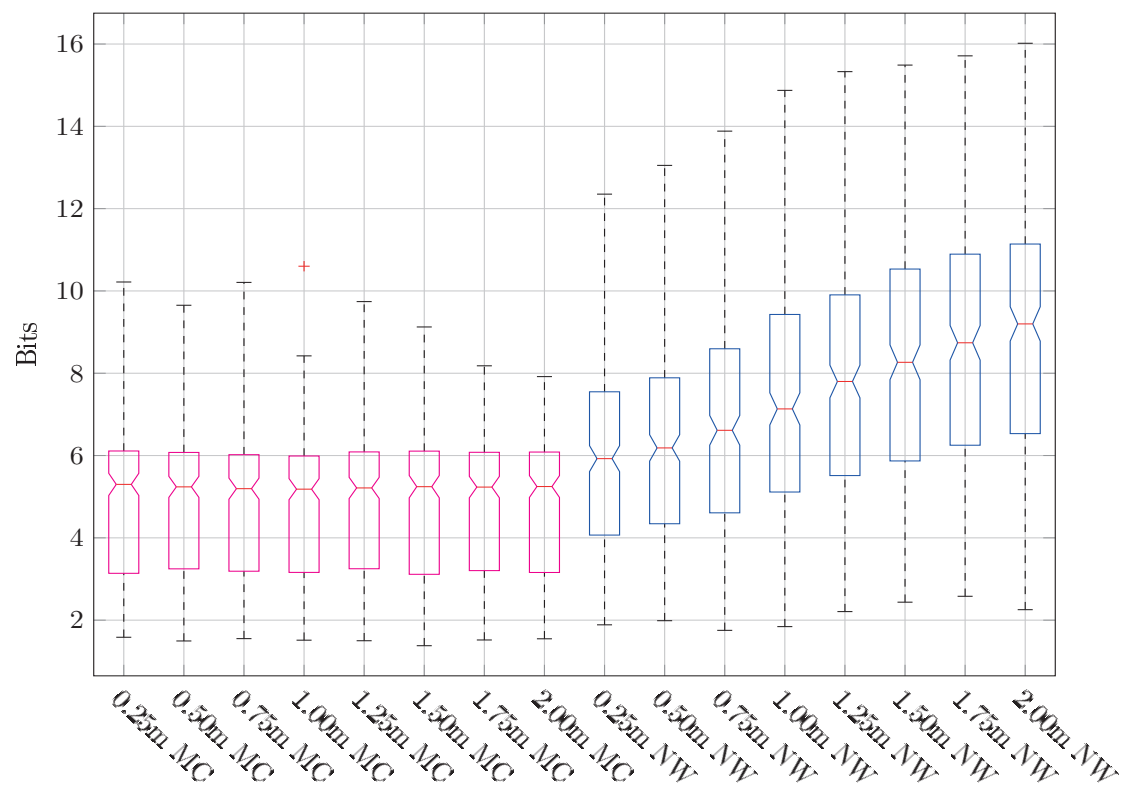

Figure 4.26: Divergence of fit between the original and estimated distribution with respect to kernel size and densification method.

these locations we have densified the map of airflow in the environment with MC and NW imputation, respectively.

The wind data set [8] does not provide ground truth because there are no denser measurements to compare. This dataset will instead be evaluated qualitatively using stability maps. The stability map shows which regions contain similar distributions representing wind flow over multiple sessions. This might lead to counter-intuitive results. Namely, regions, where the turbulent flow remains turbulent through the data set, will be marked as stable, while the areas where a laminar flow changes any of the parameters between the data sets will be marked as unstable. The method of computation of stability map is described in Sec. 4.5.

Fig. 4.27a shows a map densified with MC. The colour corresponds to the wind direction. It is possible to distinguish areas with different wind directions. Moreover, there are clear borders between areas with different airflow directions.

Fig. 4.28a shows the result of densification with NW. The areas with similar motion directions are as visible as in Fig. 4.27a, however, in this case, it is important to mention that the borders separating the regions are not so visible. We can observe a gradual, smooth change between the flows. 


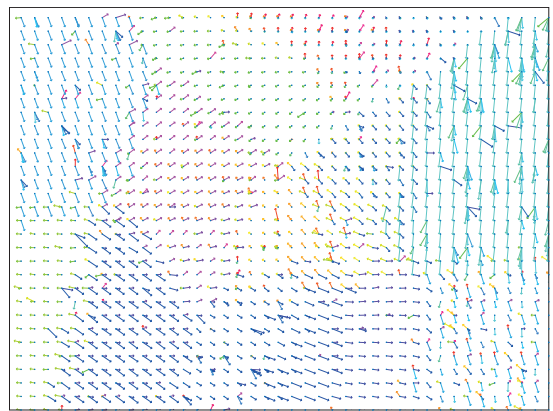

(a) A map densified with MC.

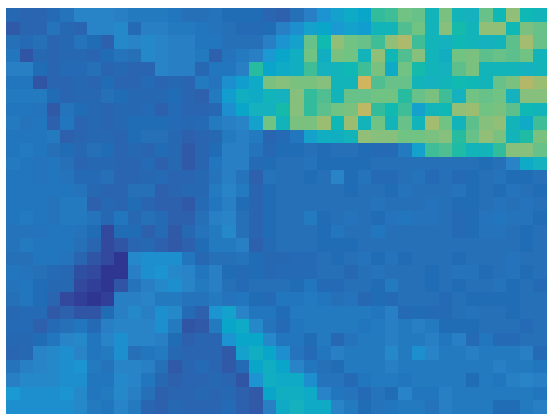

(b) A stability map for map densified with MC.

Figure 4.27: Wind map Denisfied with Monte Carlo Method for Foundry data set.

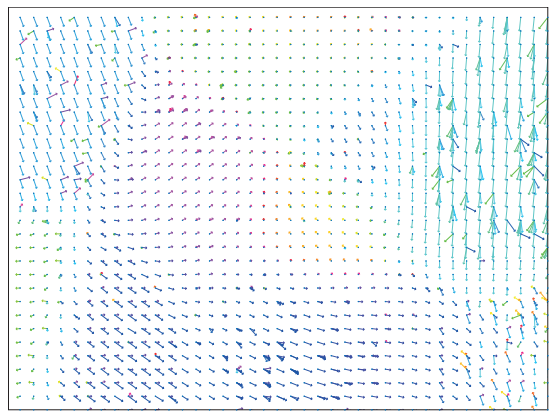

(a) A desification of the map with NW method.

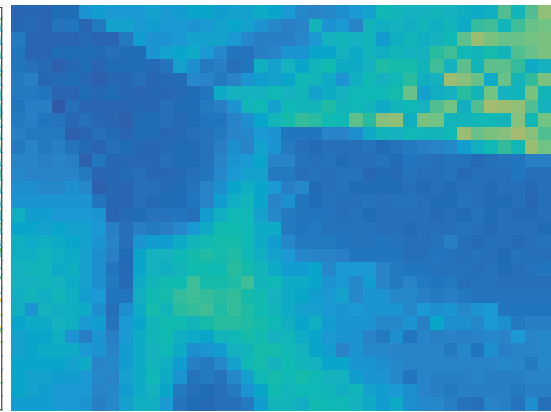

(b) A stability map for Nadaraya Watson dense map.

Figure 4.28: Wind map densification with Nadaraya Watson Method

At the beginning of this section the concept of stability maps was discussed; i. e., a map that provides information on where the wind-flow remains constant over time and where it varies.

In Fig. 4.27b and Fig. 4.28b we can see stability maps built from data sets obtained during 7 different measurement sessions. Even though the maps were obtained using different interpolation methods, large parts of them present the same level of stability. However, what is most interesting are the areas with different stability levels. We can see that in Fig. 4.27b the centre of the map is stable while in Fig. 4.28b this same area has a worse score. It is caused by the differences between the imputation methods. 
MC keeps all virtual samples; therefore it is able to model a turbulent wind behaviour. NW, on the other hand, averages the virtual observations finding the dominant wind direction. This results in different types of distributions in this area. MC builds a set of models of turbulent flow which are more similar, while NW builds a set of models which have a clear dominant direction. In the latter case, such smoothed out distributions can vary a lot between the different datasets, therefore, worsening the stability score. It depends on the application, which behaviour is more preferable.

\subsubsection{Guidelines for CLiFF-map Building}

In the process of CLiFF-map building from observations the key design choice is to pick the suitable clustering method. In Sec. 4.6.2 I present how different clustring methods impact the resulting SWGMM. Depending on the initialisation method different level of details can be obtained. On one end of the scale, there is an Isotropic MS, which provide a high number of cluster discovering multiple clusters in the data set. On the other end of the spectrum, there is OPTICS which provide information about the dominant direction in the data set.

In the densification process, the primary impact on the quality of the resulting map has the size of the kernel. Too big kernel lead to smoothing out the final map, while too small kernel will result in a prevalence of rapid changes in the flow model.

\subsection{Conclusions}

The primary objective of work presented in this chapter is to present a method capable to model motion patterns, given sparse and noisy data. To address this objective, a local method representing motion patterns as a multimodal distribution of velocities was devised. Such a representation allows to model not only the motion directions in multimodal data sets but also incorporate information about the noise into the model. To address the problem of data sparsity I have presented two methods based on a concept of imputation to build a dense map from spatially sparse observations.

\subsubsection{Summary}

The primary advantage of Circular Linear Flow Field map is flexibility. It can be used to model different phenomena, i.e. flow of people or turbulent airflow. Furthermore, the representation is not limited to underlying grid map like CT-map, but the distributions can be placed in arbitrary locations and model motion pattern for an arbitrary part of the environment.

Furthermore, the representation not only compresses the data describing the motion patterns but also extract information regarding the motion pattern. The 
mean values of Semi Wrapped Gaussian Mixture Model correspond to dominant directions of motion pattern. Furthermore, the covariance matrix for each mode describes the disparity in orientation and speed. The covariance matrix also explicitly model the dependency between the speed and orientation for a given mode. Finally, the mixing factor models a relative intensity of motion in a given direction.

It is also that the presented method is robust against incomplete data. Thanks to its locality it can model motion patterns in observed locations. Incomplete data are especially difficult to be addressed in trajectory mapping.

Moreover, CLiFF-map is not only able to build partial maps relying on incomplete data. In my work, I have also introduced mechanisms to regenerate missing data and use them for estimating motion patterns in unobserved locations.

Finally, the presented method substantially improves the performance of motion planner. However, in contrast to already existing methods (combining information about motion patterns with motion planning), CLiFF-map preserves the multi-modal characteristic of motion pattern and provides this information explicitly for the motion planner.

\subsubsection{Limitations}

CLiFF-map has numerous advantages summarised in the previous section. However, it still suffers from two types of limitations. First, which are presented in this section, are inherent for this representation and origin in from its design. Second, which are presented in the following section, are yet to be developed features.

In the previous section I have pointed on the locality as an advantage; however, in some cases, it might also be a limitation. CLiFF-map is incapable to explicitly model global motion patterns, such as streams connecting sources and sinks in the environment. Furthermore, in contrast to work presented in the previous chapter, CLiFF-map is not able to model the conditional dependencies among distributions. It can increase the ambiguity of the representation in areas where can be observed multiple intersecting motions.

Moreover, CLiFF-map introduces a strong assumption that velocities are following a normal distribution in orientation and speed. In some cases, it might lead to degradation of a model, especially in cases when the distribution is asymmetric.

Finally, the proposed distribution is discrete. That means the map does not contain information about the motion patterns in between two observed locations. However, one of the methods to estimate the distribution at the location in question is to use the densification procedure. 


\subsubsection{Future Work}

In this section, I list possible development directions of CLiFF-map.

On-line learning Currently CLiFF-map is build using an off-line algorithm. Therefore, whenever new data is obtained, it is necessary to recompute the distributions. This is a time-consuming process, which also requires storing all previous observations. Therefore one of next steps is to develop a method for online learning, which will be capable to iteratively update the distributions of the map, without storing previous observations.

Informed prior CLiFF-map relies only on the accumulated observations. However, considering expert knowledge, it should be possible to build a prior map based only on the shape of the environment. Once such a method is devised, it can be combined with online learning for refinement. In this way, the deployment time of a robotic system will be substantially shortened, and the learning process will be just a part of a standard every-day operation.

Patterns in temporal domain In the current version of CLiFF-map there is no way to map the changes of the dynamics in regular cycles. As I have already mentioned in the case of CT-map and T-CT-map information about periodic changes in flow is important to properly model current dynamics. The most challenging aspect of this task is to find a way to build a memory efficient model capable to continuously model the changes in the flow. The other interesting research question is what method would be the best to detect such patterns in a two-dimensional incomplete signal.

Event dependent patterns In addition to cyclic changes in some cases, the changes in the flow might be triggered by a particular event (e.g. arrival of an aeroplane will affect the flow of people in the terminal). For such cases, it is important to learn how long such a change will affect the flow and how far the influence will reach.

Spatial Information for Densification In my work, I have presented one approach how for taking the shape of the environment into account when building dense maps. However, this aspect requires more thorough investigation.

So far, in work presented in this chapter, there was an implicit assumption that there are no obstacles within the environment, such assumption is valid only in very few cases. Therefore, in this section, I will discuss a possible way to incorporate the information about the shape of the environment into the map densification process.

The shape of the environment has an important impact on the flow in the environment. The obstacles interrupt the flow and isolate the streams from 


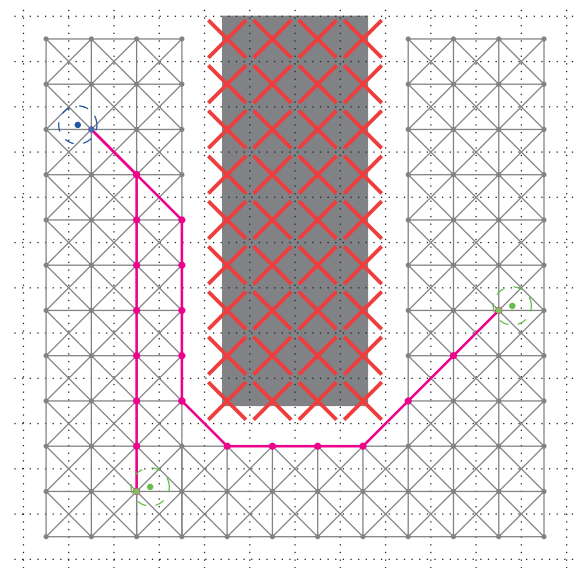

Figure 4.29: Example of graph used for distance computation. The red crosses mark non-traversable cells. The grey dots and lines represent the graph nodes and edges. The blue mark represents the observed location. The green dots represent locations to estimate. The magenta line, marks the shortest path between the observed and unobserved locations.

each other, e.g. it is very unlikely that stream will go through an obstacle. In this section, I propose a modification for a distance metrics used in the densification process so it will account for the presence of the obstacles.

The core idea is to replace the Euclidean distance with a metrics accounting for obstacles. Therefore, the proposed distance metrics is being defined as the shortest path between the nodes of the graph as shown in 4.29. As we can see, the resolution of the graph does not depend on the position of the points of interest (observed locations and estimated locations), the granularity can be chosen independently. For the cases when the nodes of the graph do not align with the points of interest the distance is being computed between the closest nodes of the graph. The rest of the densification algorithm remains unchanged.

To present the benefits of incorporating the spatial information in the densification process, I have built a simple test environment (see Fig. 4.30a) in simulation software Pedsim [72]. In the environment (see Fig. 4.30b) the agents are moving clockwise, so the wall in the middle separates the flow in the opposite directions. In Fig. 4.30c we can see a plot of distances between the red marked cell and all the other cells in the map. The darker the colour, the longer the distance. The obstacles and the isolated part of the environment are marked black. We can see that the obstacle in the middle is deforming the distance filed. Even though the area on the other side of the wall is closer according to Euclidean distance, the used metrics depicts it as the furthest reachable point. 


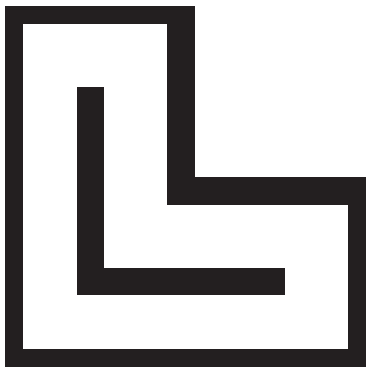

(a) Binary occupancy map of the test environment.
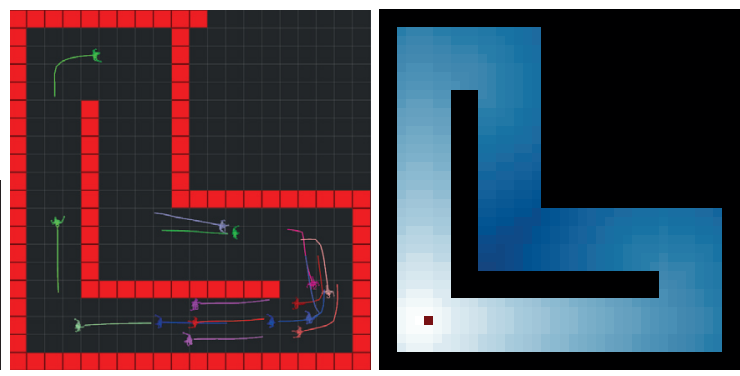

(b) Test environment with (c) Map of distance between agents in simulation soft- the point in left bottom corware. ner and all the other locations.

Figure 4.30: Test environment used to present the impac of the obstacles on desification of CLiFF-map of human flow.

In Fig. 4.31a we can see the original dense map. We can see the mapped flow of agents circulating the obstacle in the middle. Fig. $4.31 \mathrm{~b}$ shows a sub-sampled version of the original map which will be used as an input for densification procedure.

Fig. 4.31c and Fig. 4.31c show the results of map densification with NW and MC imputation respectively using unmodified distance metrics. The first observation is that the obstacles are not being respected in the dense map. In the case of NW (Fig. 4.31c) the whole map is being populated with distributions, we can see them outside of the closed circumference of the L-shaped corridor, and also intersecting with the obstacles. Moreover, the closer look at the wall separating the flows shows that in the densification process the information regarding the flow is used across the wall. We can observe a similar behaviour also for MC method (Fig. 4.31d).

In the case of MC method, even though the presence of obstacles is not being respected, not the whole map is populated with the distributions. The lack of the distributions outside of the L-shaped environment is a result of the size of the kernel. The kernel defines the probability with which the locations are going to be sampled. Therefore, if the locations containing observations are too far, then they have close to none impact on the estimated location. However, a closer look reveals that this is not enough to prevent putting distributions inside the obstacles. Similarly, to the map built with NW also for MC case, we can observe the influence of observed locations on unobserved locations across the wall.

In Fig. 4.31e and Fig. 4.31f we can observe the results of map densification considering the spatial information. The first significant difference is the fact that now distributions are not being put inside or nearby of the obstacles. The 
distance from the obstacle is a combination of the resolution of the grid and the resolution of the occupancy map. Moreover, in both cases, we can observe a smaller impact of distributions across the walls. However, in both cases, we still can observe some artefacts especially in the flow on the left side of the wall. These artefacts representing the tangent flow are results of the sparsity of the underlying data. Namely, the remaining distributions do not provide enough information to correctly estimate the change in the flow direction in this particular locations. The downside of the presented approach is the fact that the distance between the locations is being measured as the shortest path between the locations, not the shortest distance. This can lead to the deformations such as the one visible in the left bottom corner of the map built with MC method.

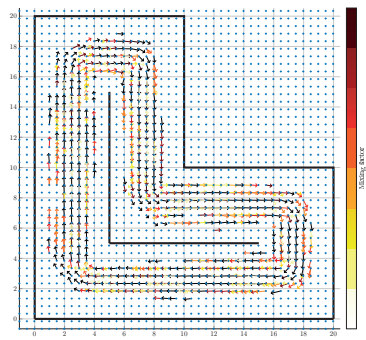

(a) Dense map of human flow.
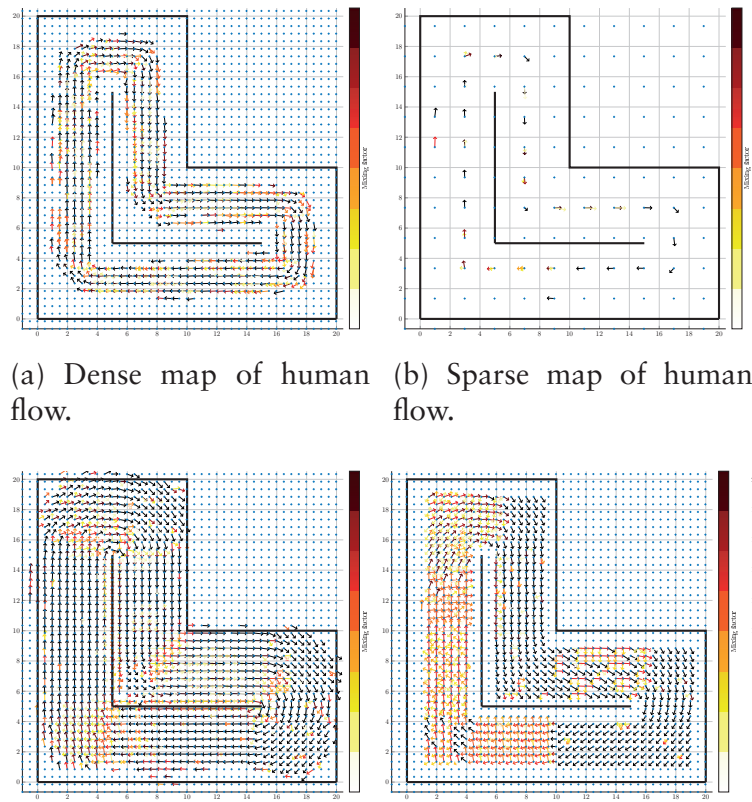

(b) Sparse map of human flow.

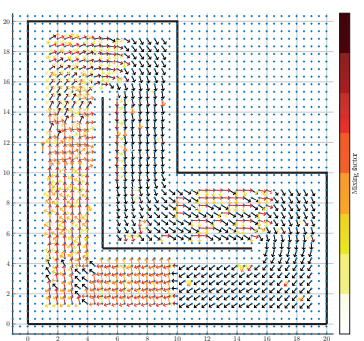
(d) Densification with MC (e) Densification with NW
without spatial informa- with spatial information. tion.

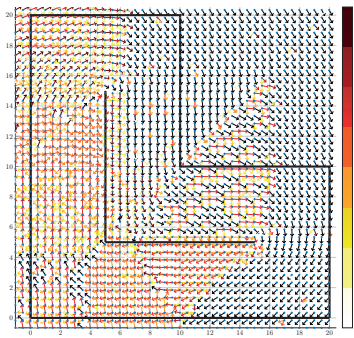

(c) Densification with NW without spatial information.

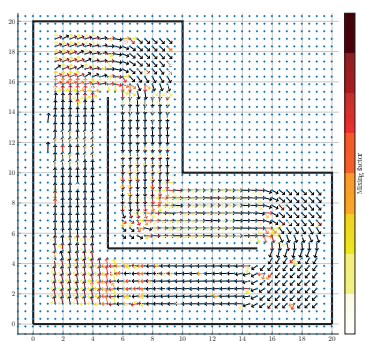

(f) Densification with $\mathrm{MC}$ with spatial information.

Figure 4.31: Comparison of densification of flow map. 


\section{Chapter 5 Application of CLiFF-map in
Motion Planning}

The focus of my thesis is to develop representations able to map motion patterns from incomplete and noisy data. In the previous two chapters I have presented, evaluated and discussed three representations CT-map, T-CT-map, CLiFF-map. However, the main purpose of any representation is to provide information for other parts of the system. In this chapter, I present work on building a motion planner which can generate a flow compliant trajectories. The planning approach presented in this chapter accounts for the typical motion of dynamic objects or continuous media such as pedestrian flows or air/water currents, modelled with CLiFF-map. The work presented here was done in collaboration with Luigi Palmieri of the University of Freiburg and has been published at IEEE International Conference on Robotics and Automation [60].

\subsection{Background}

\subsubsection{Kinodynamic Motion Planning}

Donald et al. [23] define the problem of kinodynamic motion planning as synthesising robot motion subject to kinematic and dynamic constraints simultaneously. The kinematic constraints refer to avoiding obstacles while dynamic constraints are bounds on velocity, acceleration and force. The problem of kinodynamic motion planning allows incorporating safety directly into the problem of motion planning. It happens by including a speed dependent obstacle avoidance margin in the problem parameters. Unfortunately, this came at a cost, as Xavier shows in his $\mathrm{PhD}$ thesis [78] the problem of kinodynamic motion planning is NP-hard. The CLiFF-RRT* approach is a planner that solves the kinodynamic planning problem on a field of Gaussian mixtures. Instead of using discrete search algorithms (i.e., A*, Dijkstra or similar), the approach modifies a sampling-based motion planner to better tackle the kinematic and 


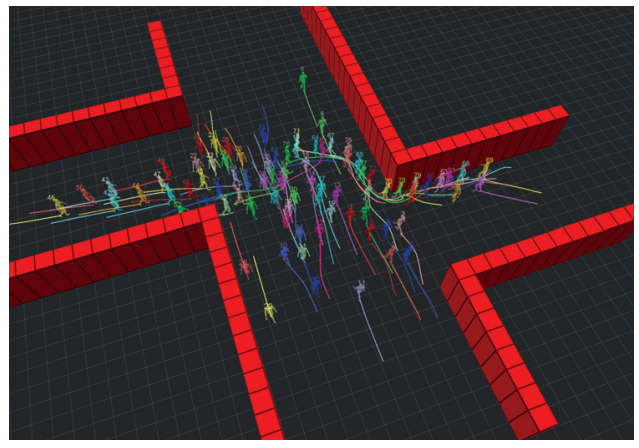

(a) The figure shows an example from the studied simulated intersect scenario where multiple flows of people encounter each other.

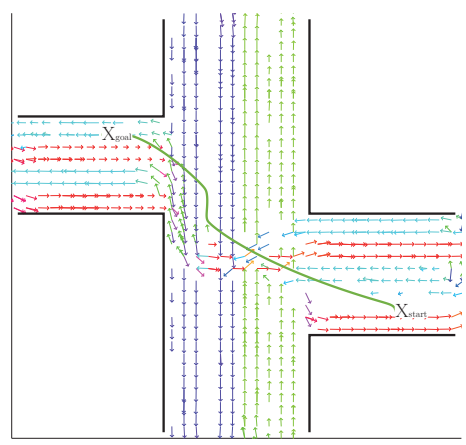

(b) An example path (in green) generated among the CLiFF-map.

Figure 5.1: An example of a flow compliant trajectory generated with motion planning approach using a cost function derived from CLiFF-map.

dynamic constraints of the robot. Particularly this choice allows the planner to generate trajectories that also satisfies velocities of the flows learned via the CLiFF representation. Using A* or Dijkstra would result in geometric paths which would need to be re-evaluated (most likely also modified) when computing also the accompanying velocity profile.

\subsubsection{Planning in Vector Fields}

Past approaches have considered motion planning over regular (unimodal) vector fields: Kularatne et al. [39] present a graph-based approach that generates time optimal and energy efficient motion plans for autonomous surface and underwater vehicles in time-varying flow fields. The kinematic constraints of the vehicles are accounted for in the cost function. Otte et al. [56] describe a graphbased algorithm to solve the problem of real-time path planning in time-varying wind fields. The anytime algorithm finds an $\alpha \beta$ solution quickly which is then, given more time, incrementally improved. Lolla et al. [42] generate paths for swarms of underwater vehicles over dynamic water flow fields using a level set approach. The method, based on a 2D grid representation of the environment, finds time-optimal paths while respecting the kinematic constraints of the system. Ko et al. [34] present an Rapidly-exploring Random Tree (RRT) based path planner over a vector field defined in the configuration space. To find a path, the algorithm tries to minimize an upstream criterion which quantifies the control effort to go against a vector field. Tree growth is guided by these criteria resulting in extensions that are more probably aligned with the vector field directions. 
Recurring patterns of human motion that people typically follow in an environment have been learned and used for planning in a number of papers $[10,26,55,63]$. Such patterns can be seen as sparse vector fields as they are only defined in parts of the state space where humans have repeatedly been observed. Based on the Risk-RRT algorithm [26], Rios et al. [63] use Gaussian processes (GP) to predict the motion of humans and generate paths with an RRT-based planner that minimise the risk of disturbing and colliding with surrounding people. O'Callaghan et al. [55] present a method that generates paths by following learned motion patterns of people using GPs. The method computes a navigational map based on the motion patterns whose cells incorporate velocity vectors. The robot navigates through the environment by querying the learned map and obtaining the next direction to follow. Bennewitz et al. [10] learn a collection of human motions patterns using Gaussian mixtures and Expectation Maximisation (EM). For each observed human, the most likely pattern is determined and used to compute motion predictions for planning. The method uses $\mathrm{A}^{*}$ on a $2 \mathrm{D}$ grid with cell costs discounted by the probability that a person is in a cell at a given time.

The contribution of this chapter is twofold. Unlike [34, 39, 42, 56] it uses CLiFF-map, which is a more powerful probabilistic representation than vector fields. In addition to the model to represent environment dynamics, and contrarily to the previously described approaches that use discrete search, the work presented in this chapter utilises an asymptotically optimal samplingbased motion planner that implicitly considers the robot's kinematic and its non-holonomic constraints by using a steer function to plan in a continuous state space.

\subsection{CLiFF-RRT*}

\subsubsection{The Algorithm}

For planning, RRT* is chosen and modified as a natural choice for optimal motion planning under kinodynamic constraints. Let $X \in \mathcal{R}^{\mathrm{d}}$ be the configuration space and $\mathcal{U} \in \mathcal{R}^{\mathrm{m}}$ the control space, the dynamics of the robot can be described by the differential equation $\dot{\mathbf{x}}(t)=f(\mathbf{x}(t), \mathbf{u}(t)), \mathbf{x}(0)=\mathbf{x}_{0}$, with $\mathbf{x}(t) \in \mathcal{X}, \mathbf{u}(\mathrm{t}) \in \mathcal{U}$ and $\mathrm{f}$ describing the system's kinematic constraints.

RRT $^{*}[31]$ is a probabilistically complete single-query sampling-based planner that asymptotically finds optimal solutions for a motion planning problem. Given an obstacle space $X_{\text {obs }} \in X$, a free space $X_{\text {free }} \in X \backslash X_{\text {obs }}$, a start state $\mathbf{x}_{\text {start }} \in X_{\text {free }}$ and a goal state $\mathbf{x}_{\text {goal }} \in X_{\text {goal }} \subset X_{\text {free }}$, the algorithm expands into $X_{\text {free }}$ a tree $\tau$ whose edges are trajectories $\sigma_{i}$ (with $\sigma_{i}(j)$ being state $j$ of trajectory $i)$ that satisfy the kinematic constraints of the considered system.

In summary, the task is approached as a hierarchical motion planning problem in first a discrete path $\mathbf{P}_{\mathcal{D}}$ that selects mixtures at relevant locations is generated, and then mixtures are used to bias the sampling and rewiring proce- 


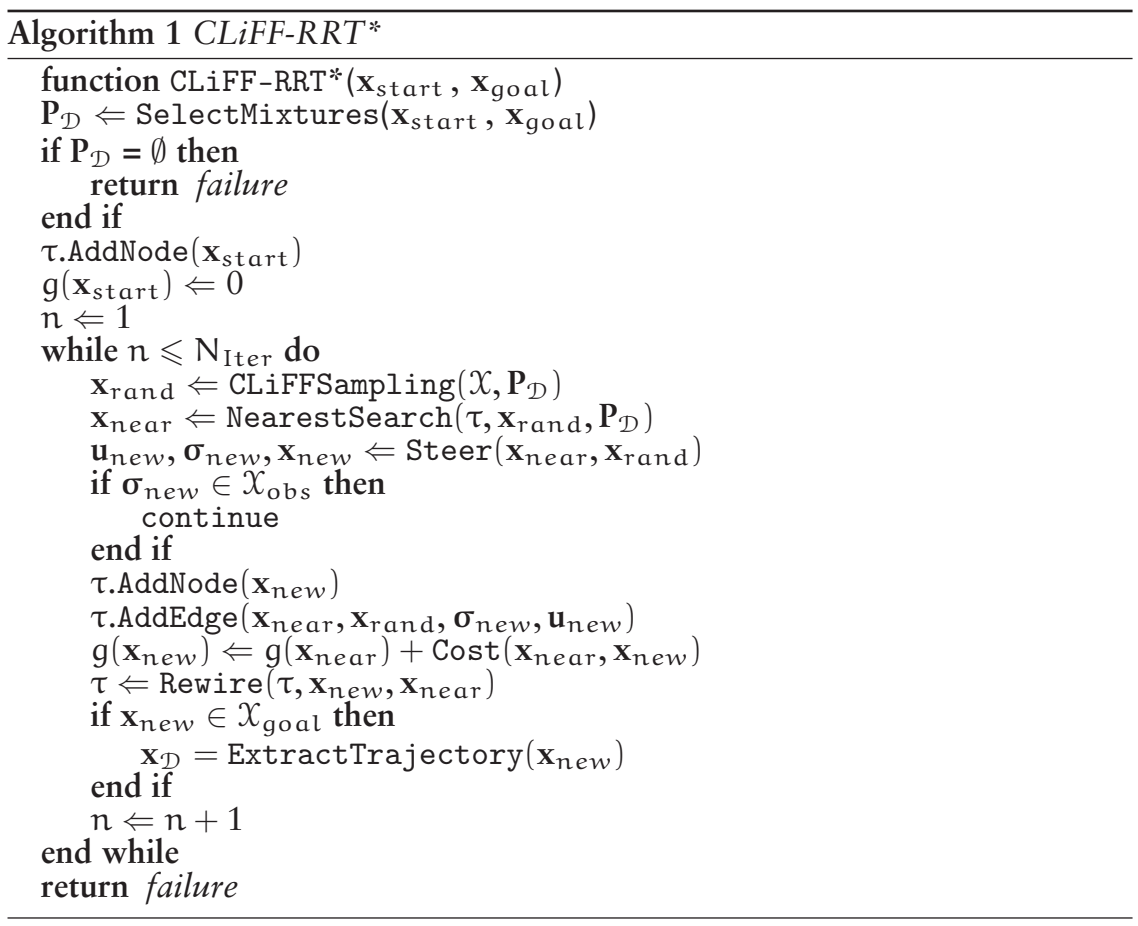

dures in RRT*. The first step makes sure that an initially feasible path is found quickly given a CLiFF-map while the second step, generates and incrementally improves a trajectory $\mathbf{x}_{\mathcal{D}}$ that satisfies the kinodynamic vehicle constraints. The result is CLiFF-RRT* in Alg. 1 whose steps are explained next.

SelectMixtures $\left(X, \mathbf{x}_{\text {start }}, \mathbf{x}_{\text {goal }}\right)$ : in this step the algorithm selects the semi-wrapped mixtures $\mathcal{N}^{S W}$ that allow the system to move from $\mathbf{x}_{\text {start }}$ to $\mathbf{x}_{\text {goal }}$ while respecting the learned environment's dynamics. Dijkstra search is run over the graph $\mathcal{G}$ in which each node $n_{\mathcal{G}, i}$ is associated to each mixture component $\left(\forall\left(\boldsymbol{\xi}_{i}, \boldsymbol{l}_{i}\right) \in \mathcal{D}\right)$ : for each map cell $\boldsymbol{l}_{i}$ edges that go between all pairs of the SWGMM components of $\boldsymbol{l}_{i}$ and those of the locations in its 4neighborhood are computed. To each edge $e\left(n_{\mathcal{G}, i}, n_{\mathcal{G}, j}\right)$, with $n_{\mathcal{G}, i}$ and $n_{\mathcal{G}, j}$ being two neighbouring nodes, the following cost is associated:

$$
c\left(e\left(n_{\mathcal{G}, i}, n_{\mathcal{G}, j}\right)\right)=d\left(e\left(n_{\mathcal{G}, i}, n_{\mathcal{G}, j}\right)\right)+u_{d}\left(\mathbf{x}_{n_{\mathcal{G}, j}}, \xi_{n_{\mathcal{G}, j}}\right)
$$

where $d\left(e\left(n_{\mathcal{G}, i}, n_{\mathcal{G}, j}\right)\right)$ is the squared Euclidean distance between the nodes, $\mathbf{x}_{n_{g, j}}$ and $\boldsymbol{\xi}_{n_{g, j}}$ respectively the state and the mixture associated to the node $n_{\mathcal{G}, j}$. 
The search generates a concatenation $\mathbf{P}_{\mathcal{D}}$ (i.e. a path) of $\mathbf{N}_{\mathbf{P}_{\mathcal{D}}}$ CLiFF-map tuples $\left(\boldsymbol{\xi}_{i}, \boldsymbol{l}_{\mathbf{i}}\right.$ ) from $\boldsymbol{l}_{0}$ (with $\mathbf{x}_{\text {start }} \in \mathbf{l}_{0}$ ) to $\mathbf{l}_{\mathrm{N}_{\mathbf{p}_{\mathcal{D}}-1}}$ (with $\mathbf{x}_{\text {goal }} \in \mathbf{l}_{\mathrm{N}_{\mathbf{p}_{\mathcal{D}}-1}}$ ) which is forwarded to the sampling unit.

CLiFfSampling $\left(X, \mathbf{P}_{\mathcal{D}}\right)$ : draws $\mathbf{x}_{\text {rand }}$ samples in $X$. The parameter $\alpha \in$ $[0,1]$ sets the probability of the biasing towards the $\mathrm{N}_{\mathbf{P}_{\mathcal{D}}}$ CLiFF-map mixtures $\xi_{i}$ of $\mathbf{P}_{\mathcal{D}}$ :

$$
\mathbf{x}_{\text {rand }} \sim \sum_{i=0}^{N_{\mathbf{P}_{\mathcal{D}}}-1} \sum_{j=1}^{J_{i}} \pi_{j} \mathcal{N}_{\xi_{i}}^{S W}\left(\boldsymbol{\mu}_{j \xi_{i}}, \Sigma_{j} \xi_{i}\right)
$$

With a probability of $(1-\alpha)$, samples are drawn from a uniform distribution defined on the entire state space $X$.

NearestSearch $\left(\tau, \mathbf{x}_{\text {rand }}, \mathbf{P}_{\mathcal{D}}\right)$ : returns the node $\mathbf{x}_{\text {near }}$ that connects to $\mathbf{x}_{\text {rand }}$ with minimum cost-to-go $C\left(\mathbf{x}_{\text {near }}, \mathbf{x}_{\text {rand }}, \mathbf{P}_{\mathcal{D}}\right)$ within distance $\delta R$ (as parameter) from the latter:

$$
\mathbf{x}_{\text {near }}=\arg \min _{\mathbf{x} \in X_{\delta R}} g(\mathbf{x})+\operatorname{Cost}\left(\mathbf{x}, \mathbf{x}_{\text {rand }}\right)
$$

with $g(\mathbf{x})$ being the cost-to-come to vertex $\mathbf{x}$ from root $\mathbf{x}_{\text {start }}$ through the current tree $\tau$. If no nodes are found within this distance, the closest vertex in terms of Euclidean distance is returned.

$\operatorname{Cost}\left(\mathbf{x}_{i}, \mathbf{x}_{\mathbf{j}}\right)$ : returns the cost of the trajectory $\boldsymbol{\sigma}$ that connects node $\mathbf{x}_{i}$ to node $\mathbf{x}_{\mathbf{j}}$. The algorithm aims to find trajectories which are smooth and short, respect the environment dynamics and minimize the upstream criteria (the control effort to move with the vector field) with respect to the off-line learned mixtures $\forall \xi_{i} \in \mathcal{D}$. For these reasons the following cost function is used:

$$
\begin{aligned}
\mathrm{C}\left(\mathbf{x}_{\mathrm{p}}, \mathbf{x}_{z}, \mathbf{P}_{\mathcal{D}}\right)= & \sum_{i=1}^{N_{p}}\|\boldsymbol{\sigma}(i)-\boldsymbol{\sigma}(i-1)\|+\left|\left(1-\left|\mathbf{q}_{i} \cdot \mathbf{q}_{i-1}\right|\right)\right| \\
& +\sum_{i=1}^{N_{p}} u_{d}\left(\boldsymbol{\sigma}(i), \xi_{i}\right)
\end{aligned}
$$

where $\boldsymbol{\sigma}(i)$ are intermediate states of the trajectory $\boldsymbol{\sigma}$ connecting $\mathbf{x}_{\mathbf{p}}$ to $\mathbf{x}_{z}, \mathbf{q}_{i}$ are related quaternions, and $U_{d}\left(\sigma(i), \xi_{i}\right)$ being the upstream functional value at $\sigma(\mathfrak{i})$. A supervised learning approach can be used to improve the efficiency of the cost computation as in [57].

Steer $\left(\mathbf{x}_{i}, \mathbf{x}_{\mathbf{j}}\right)$ : generates a trajectory $\boldsymbol{\sigma}$ and the set of controls $\mathbf{u}$ needed to steer the system from $\mathbf{x}_{i}$ to $\mathbf{x}_{j}$. The analytical steer function connects any pair of states and respects the topological property as described in [31, 59].

Rewire $\left(\tau, \mathbf{x}_{\text {new }}, \mathbf{x}_{\text {near }}\right)$ : rewires the tree $\tau$ as in the original RRT* [31], using the above described Steer and Cost functions. The rewiring is done at each iteration on a set of vertices found by a near neighbor search as in [32]: 
it finds the set of all the states in $\tau$ that lie within a box centered on $\mathbf{x}_{\text {near }}$ whose volume scales as $\gamma^{\mathrm{D}} \frac{\log (\mathfrak{n})}{n}$, with $\mathrm{D}$ being the Hausdorff dimension of the distribution generated by the system dynamics.

\subsubsection{Extended Upstream Criterion}

The goal of CLiFF-RRT* is to find planning solutions that trade off classical motion planning metrics such as path length and path smoothness with the compliance with the environment dynamics. To quantify the latter, the upstream criterion, proposed by Ko et al. [34] for unimodal vector fields, is here extended to fields of Gaussian mixtures to account for the multi-modal nature of the CLiFF-map representation.

Given a state $\mathbf{x}_{i}$ that falls into the vicinity of location $\boldsymbol{l}_{i}$ to which a mixture $\xi_{i}$ with $J_{i}$ semi-wrapped normal components is associated, the upstream metric is computed as

$$
\mathrm{u}_{\mathrm{d}}\left(\mathbf{x}_{i}, \boldsymbol{\xi}_{\mathrm{i}}\right)=\sum_{j=1}^{\mathrm{J}_{\mathrm{i}}}\left(\left\|\boldsymbol{\mu}_{\mathrm{ji}}\right\|-\left\langle\boldsymbol{\mu}_{\mathrm{ji}}, \mathbf{x}_{\mathrm{i}}^{\prime}\right\rangle\right)
$$

with $\langle$,$\rangle being the inner product, \boldsymbol{\mu}_{j i}$ the first-order moment of the jth component of mixture $\xi_{i}$, and $\mathbf{x}_{i}^{\prime}$ the unit vector describing the direction of the path at $\mathbf{x}_{i}$. When $\mathbf{x}_{i}$ is associated with location $\boldsymbol{l}_{\boldsymbol{i}}$ that has no distributions, a nearest-neighbour interpolation is performed and $\mathrm{U}_{\mathrm{d}}\left(\mathbf{x}_{i}, \boldsymbol{\xi}_{i}\right)$ is computed using the closest mixture $\boldsymbol{\xi}_{i}$. The criterion yields low costs for paths that comply with the directions of CLiFF-map mixture components and high costs for paths in opposite directions.

\subsubsection{Steer Function}

Let us consider wheeled mobile robots with a differential drive kinematic configuration with state $\mathbf{x}=(x, y, \theta, v)$. The $(x, y) \in \mathbb{R}^{2}$ is the Cartesian position, $\theta \in[-\pi, \pi)$ is the heading orientation and $v$ its translational velocity. After a Cartesian-to-polar coordinate transformation, the equations of motions are $\dot{\rho}=-\cos \alpha \nu, \dot{\alpha}=\frac{\sin \alpha}{\rho} v-\omega, \dot{\phi}=-\omega$ where $\rho$ is the Euclidean distance between the Cartesian coordinates of the robot pose $(x, y, \theta)$ and of the goal state, $\phi$ the angle between the $X$-axis of the robot reference frame $\left\{X_{r}\right\}$ and the $x$-axis of the goal state frame $\left\{X_{g}\right\}, \alpha$ the angle between the $y$-axis of the robot reference frame and the vector connecting the robot with the goal position, $v$ the translational and $\omega$ the angular robot velocity. Thanks to the polar representation it is possible to overcome the obstruction to stabilizability for such system described in the Theorem of Brockett [14]. To exactly connect any pairs of states smoothly and efficiently for this description of a wheeled mobile robot, the POSQ steer function [58] is used and extended (see Fig. 5.2). Each time when the steer function is called in Alg. 1 to connect two sampled states 


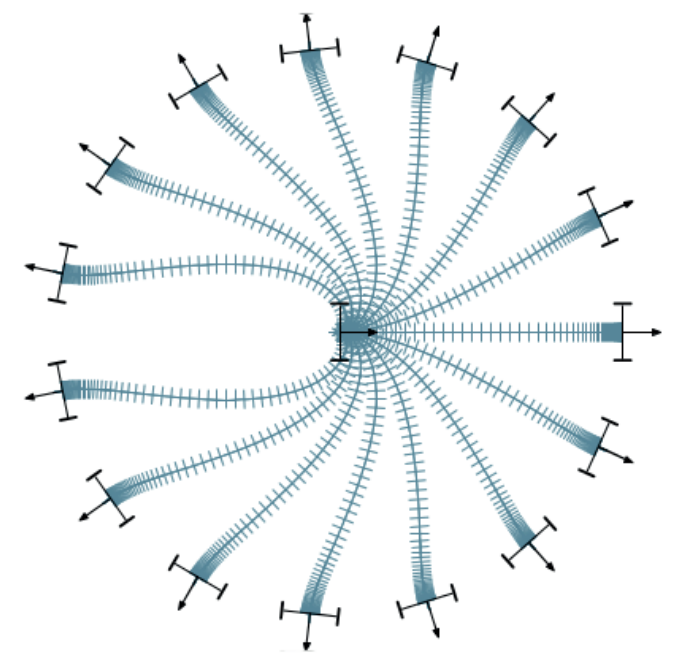

Figure 5.2: Example smooth paths generated by the POSQ steer function when steering the robot from the center to the poses on the circle.

$\mathbf{x}_{1}=\left(x_{1}, y_{1}, \theta_{1}, v_{1}\right)$ to $\mathbf{x}_{2}=\left(x_{2}, y_{2}, \theta_{2}, v_{2}\right)$, it plans an initial extension by using POSQ as described in [58] and then modifies the velocity profile so that the initial velocity is equal to $v_{1}$ and final one is $v_{2}$. The velocity profile is generated using an efficient third-order polynomial time-law [12].

\subsubsection{Algorithm Properties}

RRT $^{*}$ has favourable properties such as probabilistic completeness and asymptotic optimality. This section contains a brief analysis how the alterations of CLiFF-RRT* impact those properties. RRT and RRT* are probabilistically complete as their sampling procedure draw samples from a uniform distribution over the state space. This also applies to CLiFF-RRT* which generates, at a given probability, uniformly distributed random samples, none of which are rejected. Regarding asymptotic optimality, Karaman and Frazzoli have shown that for $n$ uniformly distributed random samples, a steer function that connects two poses exactly, an admissible cost function and a specific constant $\gamma$ in the selection of the neighboring nodes, RRT* almost surely converges asymptotically to the optimal solution as $n$ goes to infinity [32]. CLiFF-RRT* uses the same rewiring and neighbour nodes selection procedures of RRT*. It uses a steer function that exactly connects two nodes, and its cost function $\mathrm{C}\left(\mathbf{x}_{\mathfrak{i}}, \mathbf{x}_{\mathbf{j}}, \mathbf{P}_{\mathcal{D}}\right)$ is an admissible cost function for RRT ${ }^{*}$ : it is monotonic, additive and Lipschitz continuous. Moreover, it generates, at a given probability 
$(1-\alpha)$, uniformly distributed random samples. Therefore CLiFF-RRT* retains the asymptotic optimality property of RRT*.

\subsection{Evaluation}

The purpose of the experiments is to evaluate the performance of the proposed CLiFF-RRT* algorithm with respect to the baselines of regular RRT and RRT* and an uninformed variant of the algorithm, called All-Mixtures-RRT*, that generates samples from a distribution composed of all CLiFF-map mixtures and not on a subset as it the case with CLiFF-RRT*. All methods use the steer function described in Sec. 5.2.3 and cost function described in Sec. 5.2.1.

The experiments were run on a single core of an ordinary PC with a 2.80 $\mathrm{GHz}$ Intel i7 processor and $32 \mathrm{~GB}$ RAM using $\mathrm{C}++$. After a set of informal validation runs the parameters $\alpha$ to 0.95 and $\delta R$ to $4 \mathrm{~m}$ were set, while $\gamma$ is set in a way to satisfy the requirements of RRT*(See [32]).

In the experimental part, it is shown how the planners behave in environments of varying complexity. Given the interest in wheeled mobile service robots, environments are generated by exploiting off-line learned motion models of pedestrian traffic. Four simulated test environments were designed (see Fig. 5.1 and Fig. 5.3-5.4).

In all cases, there are different flow dynamics between the start and goal, and different planning solutions (homotopy classes) are possible. The $L$ and $P$ environments contain few obstacles. Here the planners have less geometric constraints to follow the upstream criterion in the free space better. The maze environment has many different homotopy classes and narrow passages: the environment has many different flows that go against each other. The intersect scenario has many flows of pedestrians coming from different corridors intersecting at a junction: also here there are few geometric constraints, but several flows are present. All the CLiFF-maps have been generated with the help of the pedestrian simulator Pedsim [72]. The grid cell size for the CLiFF-map is set to $1 \mathrm{~m}$ in all the environments.

For each planner and environment, 50 runs were performed. For the $L, P$ and intersect scenarios each run lasts $60 \mathrm{~s}$, and for the maze scenario $120 \mathrm{~s}$. The means and standard deviations of the following metrics were computed: planning time $T_{s}$ (measured in seconds), resulting trajectory length $l_{p}$ (measured in meters) and final cost $C_{s}$. Furthermore, to measure smoothness a metric introduced in work [59] was used: roughness R, defined as the square of the change in curvature $\mathrm{K}$ of the robot, integrated along the trajectory and normalised by the trajectory length L,

$$
R=\int_{t_{0}}^{t_{l}}\left|\frac{1}{L} \frac{d \kappa}{d t}\right|^{2} d t .
$$

Smoother trajectories have smaller roughness. Also, the percentage of trajectories found (problems solved) within the planning time limit was reported. 


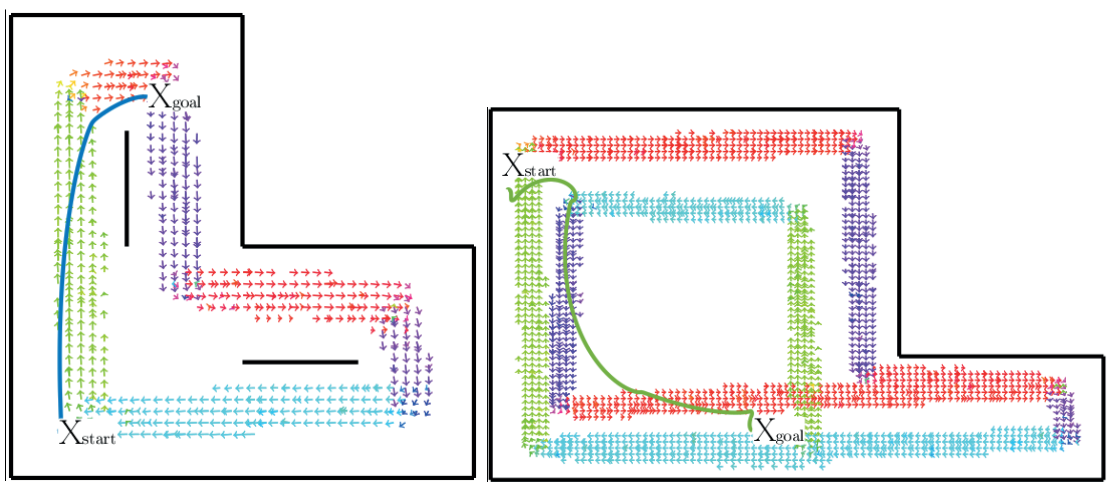

(a) An example CLiFF-RRT* path (b) An example CLiFF-RRT* path (in green) (in blue) generated in the $L$ scenario. generated in the $P$ scenario. The arrows represent the learned mixtures.

Figure 5.3: In these environments just a few obstacles are present. The algorithm finds the best solution that optimises path length and the upstream criterion: the solutions follow the learned flows.

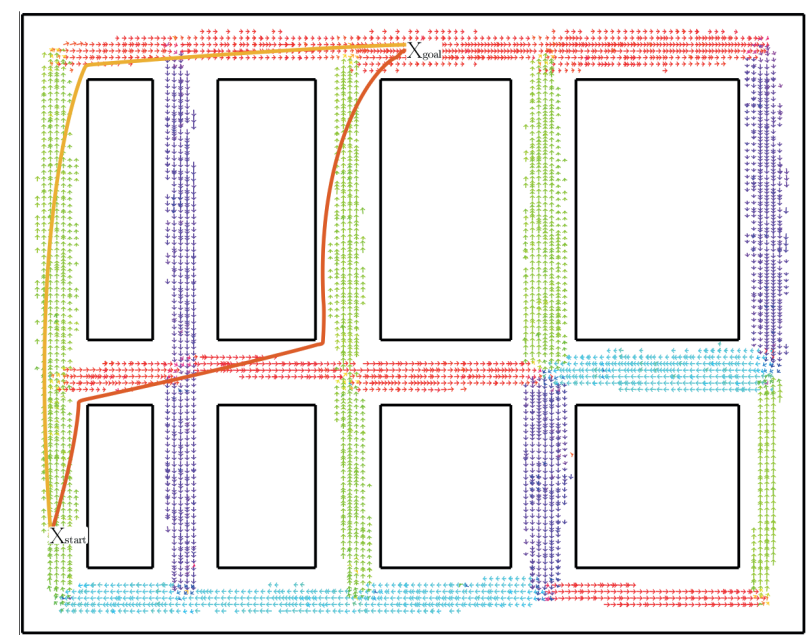

Figure 5.4: An example CLiFF-RRT* path generated in the more complex maze scenario. In red the RRT* path generated by minimising only path length. The arrows describe the learned mixtures. CLiFF-RRT* computes a path (in orange) that better minimises the upstream criterion, without encountering or crossing flows going in opposing direction. 


\subsubsection{Results}

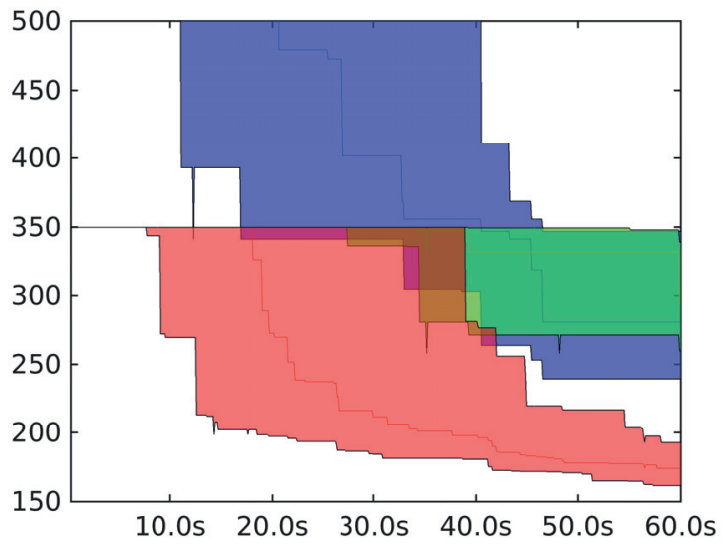

Figure 5.5: Cost convergence plot (median, first and third quartiles computed over 50 runs) respect to the planning time of CLiFF-RRT* (in red), AllMixtures-RRT* (in green) and RRT* (in blue), for the Intersect scenario. The informed sampling allows CLiFF-RRT* to quickly find an initial solution and to converge faster to a lower cost solution than the baselines.

\begin{tabular}{|l|l|}
\hline Environments & $\mathrm{T}_{\text {Dijkstra }}[\mathrm{ms}]$ \\
\hline$L$ & 4.36 \\
\hline$P$ & 3.36 \\
\hline Intersect & 2.83 \\
\hline Maze & 121.20 \\
\hline
\end{tabular}

Table 5.1: Experimental results: Planning times of Dijkstra

The experimental results for CLiFF-RRT* and the three baseline planners are given in Table 5.5. The best values are highlighted in boldface, and smaller values are better for all performance metrics excepts for the percentage of the problems solved.

CLiFF-RRT* outperforms the baselines with respect to all the metrics. The following observations were made:

1. CLiFF-RRT* with its focused search finds an initial solution faster than all the baselines (thus also Informed RRT* [27] which behaves as RRT* until a first solution is found). RRT and RRT* do not avoid the timeconsuming exploration of the entire state space. For this reason, the latter more often fails to find an initial solution in the given time. Moreover, Table 5.1 shows that the planning time of sub-selecting a set of mixtures with the Dijkstra search does not alter the overall planning time of 


\begin{tabular}{llllll}
\hline Planner & Cost $\mathrm{C}_{\mathrm{s}}$ & Time $\mathrm{T}_{\mathrm{s}}[\mathrm{s}]$ & Traject. len. $\mathrm{l}_{\mathrm{p}}[\mathrm{m}]$ & Roughness $\mathbf{R}$ & $\mathbf{6 0 s}$ \\
\hline CLiFF-RRT* $^{*}$ & $\mathbf{1 1 1 . 4 2} \pm 5.08$ & $\mathbf{5 . 3 0} \pm 8.08$ & $\mathbf{3 3 . 5 9} \pm 0.78$ & $0.00007 \pm 0.00004$ & $\mathbf{1 0 0 \%}$ \\
All-Mixtures-RRT & $130.89 \pm 32.92$ & $14.97 \pm 17.65$ & $35.48 \pm 2.31$ & $0.00009 \pm 0.0001$ & $48 \%$ \\
RRT & $784.82 \pm 618.5$ & $15.83 \pm 17.16$ & $40.59 \pm 7.36$ & $0.0023 \pm 0.0048$ & $36 \%$ \\
RRT $^{*}$ & $212.26 \pm 193.4$ & $28.15 \pm 14.91$ & $37.31 \pm 3.35$ & $0.00043 \pm 0.0013$ & $34 \%$ \\
\hline
\end{tabular}

Table 5.2: Experimental results: Trajectory quality and planning efficiency for $L$ environment

\begin{tabular}{llllll}
\hline Planner & Cost $\mathrm{C}_{\mathrm{s}}$ & Time $\mathrm{T}_{\mathrm{s}}[\mathrm{s}]$ & Traject. len. $\mathrm{l}_{\mathrm{p}}[\mathrm{m}]$ & Roughness $\mathrm{R}$ & 60s \\
\hline CLiFF-RRT $^{*}$ & $\mathbf{1 5 1 . 5 1} \pm 12.62$ & $\mathbf{3 1 . 1 3} \pm 32.66$ & $\mathbf{1 2 3 . 2 3} \pm 1.02$ & $0.000022 \pm 0.00004$ & $\mathbf{9 0 \%}$ \\
All-Mixtures-RRT $^{*}$ & $180.52 \pm 54.83$ & $36.74 \pm 38.12$ & $126.43 \pm 3.83$ & $0.000038 \pm 0.000025$ & $10 \%$ \\
RRT & $1260.54 \pm 1278.96$ & $41.74 \pm 17.87$ & $169.94 \pm 25.93$ & $0.00058 \pm 0.00064$ & $10 \%$ \\
RRT $^{*}$ & $560.78 \pm 397.98$ & $64.29 \pm 35.93$ & $176.68 \pm 43.67$ & $0.00053 \pm 0.00062$ & $14 \%$ \\
\hline
\end{tabular}

Table 5.3: Experimental results: Trajectory quality and planning efficiency for Maze environment

CLiFF-RRT*. Additionally, CLiFF-RRT* on average, converges faster to a lower cost solution than the baselines, see Fig.5.5. Those results confirm the intuition that having prior knowledge of the environment's dynamics, improves planning efficiency (e.g. in the experiments knowing how people usually move in an environment allows the planner to explore a smaller part of the configuration space).

2. CLiFF-RRT* finds less costly solutions if compared to all the baselines. The mixtures selected via the Dijkstra search guide the tree towards areas of the state space where the found trajectory is most likely to offer a good trade-off between length and control-effort against the dynamics of the environment (the upstream-criterion). The uniform sampling of RRT and RRT* has not such knowledge thus those planners fail to find a better solution. All-Mixtures-RRT*, without the Dijkstra search biasing, fails to find a better solution too in the allowed planning time.

3. The CLiFF-RRT* sampling strategy results in smoother trajectories than the baselines. Mainly because the off-line learned mixtures bias the tree towards concatenation of extensions with fewer velocity discontinuities. Uniform sampling generates velocities without prior knowledge about usual motions in particular portions of the state space, thus producing less correlated velocities.

\subsection{Conclusions}

CLiFF-RRT* exploits prior knowledge of the environment's dynamics in order to efficiently plan smooth and short paths. Differently, from the previous ap- 


\begin{tabular}{llllll}
\hline Planner & Cost $\mathrm{C}_{\mathrm{s}}$ & Time $\mathrm{T}_{\mathrm{s}}[\mathrm{s}]$ & Traject. len. $\mathrm{l}_{\mathrm{p}}[\mathrm{m}]$ & Roughness $\mathrm{R}$ & 60s \\
\hline CLiFF-RRT* $^{*}$ & $\mathbf{1 1 2 5 . 1 6} \pm 659.0$ & $11.42 \pm 14.11$ & $59.13 \pm 4.48$ & $0.000098 \pm 0.00025$ & $\mathbf{5 6 \%}$ \\
All-Mixtures-RRT* & $1688.09 \pm 27.48$ & $12.38 \pm 7.68$ & $103.54 \pm 31.1$ & $0.0007 \pm 0.0024$ & $2 \%$ \\
RRT & $2532.96 \pm 798.46$ & $21.16 \pm 19.8$ & $82.87 \pm 23.04$ & $0.0007 \pm 0.0014$ & $16 \%$ \\
RRT* & $1128.06 \pm 454.64$ & $25.43 \pm 18.90$ & $122.61 \pm 34.72$ & $0.00057 \pm 0.00007$ & $16 \%$ \\
\hline
\end{tabular}

Table 5.4: Experimental results: Trajectory quality and planning efficiency for $P$ environment

\begin{tabular}{llllll}
\hline ek Planner & Cost $\mathrm{C}_{\mathrm{s}}$ & Time $\mathrm{T}_{\mathrm{s}}[\mathrm{s}]$ & Traject. len. $\mathrm{l}_{\mathrm{p}}[\mathrm{m}]$ & Roughness $\mathrm{R}$ & $\mathbf{6 0 \mathrm { s }}$ \\
\hline CLiFF-RRT* & $\mathbf{1 8 2 . 5 2} \pm 28.77$ & $\mathbf{2 4 . 9 6 \pm 1 7 . 2 9}$ & $34.71 \pm 1.00$ & $0.013 \pm 0.019$ & $\mathbf{7 6 \%}$ \\
All-Mixtures-RRT* & $307.67 \pm 56.25$ & $29.4 \pm 18.70$ & $51.77 \pm 20.76$ & $0.013 \pm 0.0104$ & $14 \%$ \\
RRT & $722.15 \pm 373.35$ & $27.78 \pm 25.55$ & $41.97 \pm 10.14$ & $0.0196 \pm 0.012$ & $10 \%$ \\
RRT $^{*}$ & $298.75 \pm 69.42$ & $27.16 \pm 15.77$ & $47.61 \pm 16.39$ & $0.087 \pm 0.0069$ & $24 \%$ \\
\hline
\end{tabular}

Table 5.5: Experimental results: Trajectory quality and planning efficiency for Intersect environment

proaches, it plans considering a multi-modal representation (not a simple vector field) of the dynamic obstacles' motions. The approach is evaluated in four different environments and compared to three different baseline planners, namely RRT, RRT* and an uninformed version of our algorithm that samples considering all the off-line learned CLiFF distributions. The results indicate that the CLiFF-map priors help CLiFF-RRT* to find shorter and smoother trajectories significantly faster than all the baselines. Moreover, the results show that the approach requires less control effort (smaller cost) to drive a wheeled mobile robot through a dynamic environment. CLiFF-RRT* retains the probabilistic completeness and the asymptotic optimality of RRT*. In future work is to study the behaviour of the algorithm in different types of dynamic environments (e.g. UAVs in robot olfaction scenarios). The further work will also focus on the use of deterministic, as opposed to random sampling sequences, this can improve planning efficiency and reduce the variance of the results. 


\section{Chapter 6 Closing Remarks}

The overarching idea behind the work presented in the thesis is to investigate methods allowing to represent the variety of the real-world motion patterns in a compact yet expressive way.

\subsection{Contributions}

In this thesis I present, discuss and evaluate two representations, ConditionalTransition Map (CT-map) (accompanied by its temporal extension Temporal Conditional-Transition Map (T-CT-map)) and Circular Linear Flow Field map (CLiFF-map). Both representations aim at building probabilistic models of multimodal flows present in the environment; however, each of them has its own merits.

The focal point of the work is to develop representations that can show the flow of discrete objects or continuous media, rather than focus on the changes of the shape of the environment or learn the levels of dynamics across the environment. The motivation for such a choice of a focal point is a belief that robots should not only avoid semi-static obstacles or plan their trajectories through areas with low levels of dynamics but also use the information about flow to improve their behaviour (e.g. seamlessly follow the stream of people or use the air streams to lower energy consumption during a flight). Furthermore, the tasks of mapping changes of the shape of the environment and learning the levels of dynamics across the environment have received a substantial amount of attention over the years (see Sec. 2.2). In contrast, the problem of flow mapping has received substantially less attention.

\subsubsection{CT-map and T-CT-map}

CT-map and T-CT-map are grid based representations. However, in contrast to the previously existing grid based methods (see Sec. 2.2.1), CT-map and T-CT-map are not assuming cell independency. The methods assuming cell in- 
dependence does not consider the impact of occupancy changes among adjacent cells but model the changes in a cell occupancy in isolation from its neighbours. As a result CT-map and T-CT-map provide a way to model flow of occupancy across the environment.

Furthermore, T-CT-map also makes attempt to jointly represent the direction of flow of occupancy and speed of occupancy.

CT-map and T-CT-map model flow of occupancy across cell borders with a set of conditional probabilities. The conditional probabilities connect the exit and the entry direction of occupancy shift when something moves through a cell in the grid map. Furthermore, the fact that the representation is considering each pair of entry and exit shift separately allows preserving the multimodal character of flow of occupancy.

It is also important to mention that CT-map and T-CT-map relies only on occupancy information. This allows to build meaningful model of dynamics without using object tracking modules. Exclusion of object tracking modules makes the processing pipeline simpler and the mapping process more robust. Furthermore, it also allows to address motion of all objects using one tool without need to include multiple complex object detection and tracking methods.

Finally, thanks to CPP-tree it is possible to extract global motion patterns from CT-map and T-CT-map, which are local represnatations.

\subsubsection{CLiFF-map}

CLiFF-map is also designed to model the flow; however, in contrast to CT-map and T-CT-map it is not built on top of an already existing spatial representation, but its parameters are directly estimated based on velocity measurements. Thanks to the fact that CLiFF-map does not depend on any underlying representation it can provide velocity model for arbitrary locations on the map. Furthermore, CLiFF-map is a continuous representation, that means that it can represent arbitrary orientation and speed without being constrained by the discretisation of the representation.

The fact that CLiFF-map relies on velocity measurements substantially extends the area of its application. CLiFF-map in contrast to mapping of spatial configuration changes (see Sec. 2.2.1) and trajectory mapping (see Sec. 2.2.3), can model not only flow of discrete objects but also flow of continuous media.

CLiFF-map similarly to CT-map and T-CT-map is also able to preserve the multimodal character of the flow. To do so CLiFF-map associates to each location in the map a multimodal distribution, where each mode is corresponding to each of local motion patterns, differentiated based on the speed and orientation of motion of the objects. It is important to emphasise that by using SWGMM CLiFF-map can retain the information between the speed and orientation in local motion pattern. This feature is especially crucial while modelling microscale airflows, where a large number of modes can be observed differing in speed and orientation. 
Furthermore, CLiFF-map is accompanied by a sampling-based and kernelbased method used for building a dense map with spatially sparse measurements. This allows to build meaningful maps when there is no complete observability, and such a feature is very valuable in a majority of real-world applications.

\subsection{Limitations}

It needs to be noted that the described representations have limitations which need to be considered when integrating them into an applied system. These issues stem from the fact that the representations are local, discrete, parametric and suited only for dynamic objects or continuous media.

Local Maps built with each of the representations discussed in the thesis can be viewed as sets of models, where each model is valid only for a small part of the environment. This design makes the problem of mapping tractable, especially if faced with noisy and incomplete data. However, it also means that information regarding global motion patterns is not directly accessible. One of the ways to, at least partially, overcome this limitation is to infer global patterns based on the local models.

For CT-map and T-CT-map a partial solution for this problem is CPP-tree (a similar solution can be devised for CLiFF-map). However, CPP-tree also suffers from the local character of the representation. In the process of building a CPP-tree (see Sec. 3.3.3), each new edge is added to the tree based only on a local condition (i.e., if there is a non-zero probability of a given exit and entry shift to happen). This may lead to situations, where global motion patterns if they have overlapping parts, are merged in the process of building a CPP-tree. Such a situation can take place in a quarry, where parts of the roads are shared between wheel loaders executing load-haul-dump cycle and trucks distributing gravel to construction sites. The wheel-loaders will not exit the quarry while the trucks will not move beyond the loading point.

Discrete The problem of discretisation is closely related to the locality issue. Discretisation means that the representations do not model flow for arbitrary points in space, but only at predefined locations. Furthermore, the discretisation relies on the assumption that the model is valid for the whole area it is associated with. This assumption, however, might not always true. In order to better understand the impact of discretisation let us consider a case where a single distribution of CLiFF-map describes an area in front of two doors. The distribution consists of two modes with equal speed but with opposite directions. Based on such distribution robot can reason that it can freely enter through any door. However, because the area describes by the distribution is 
too wide (spans across both doors), the information that the left door is only for exiting while right only for entering is lost.

Parametric The representations introduced in the thesis are parametric. It introduces biases regarding how the flow looks.

In case of CT-map and T-CT-map this limitation is visible in two aspects. First, CT-map and T-CT-map consider only eight possible orientations, that causes that motion patterns which orientations differ less than $\pi / 4$ are merged. Furthermore, the assumption that durations times are normally distributed and unimodal can lead to following problems. If the durations measurements are not following a normal distribution, the estimated parameters will not reflect the true nature of the data but represent best fitting normal distribution. Furthermore, if the data is unimodal but not symmetric, the mean value is not equivalent to the dominant duration in the dataset. Finally, if the data is following a multimodal distribution, such information is not retained.

In the case of CLiFF-map some of the limitations of CT-map and T-CT-map are addressed. CLiFF-map can handle an arbitrary number of orientations and also the multimodal character of the data. However, CLiFF-map still assumes that data is normally distributed. In order to properly understand this limitation, let us consider a situation where the data is following a skewed distribution whose mode is at low speeds, but there are also some observations corresponding to faster moving vehicles. In such a case normal distribution will not be able to capture the true dominant speed neither preserve the information about the true shape of the data.

Dynamic The representations described in the thesis are tailored to model motion patterns of dynamic objects or flow of continuous media. That means they focus on building model of observed motion. In the result, the dynamics caused by semi-static objects will not be included in the final map. This lead to a situation where in order to represent all types of dynamics in the environment it is necessary to use more than one representation. This limitation comes from the fact that different types of phenomena and different types of tasks require different representations.

\subsection{Future Research Directions}

Several possible research directions could be explored in order to extend the representational power further and the applicability of MoDs for robotics.

\subsubsection{Representations Developments}

The detailed description of further development of CT-map, T-CT-map and CLiFF-map can be found in respective chapters. In this section, however, I high- 
light the two most important research directions: temporal variability of Maps of Dynamics (MoDs) and spatial dependencies of MoDs.

\section{Temporal Variability}

Dynamics usually follow stochastic non-stationary processes; in other words, the patterns of dynamics change over time. The current version the MoDs presented in the thesis are building time-ignorant models.

In order to properly address the time-dependent changes in the environment's dynamics, it is necessary to remember that there are three groups of temporal changes of dynamics and they should be handled differently.

Periodical Changes This type of changes are regularly reoccurring in time, i.e. the daily cycle of sea breeze and land breeze or alteration between morning and evening commuter traffic. While handling this type of dynamics, the aim should not be to learn the history of the changes but rather to discover the patterns governing the changes. A solution for such tasks can be integration of CT-map, T-CT-map and CLiFF-map with Frequency Map Enhancement (FreMEn) [36], which models the periodic character of events.

Event Dependent Changes This type of changes of dynamics repeats but does not follow a regular schedule. Instead, they are triggered by some irregular events, e.g. a change of traffic because of a concert in a nearby stadium. In this case, it is necessary to find methods to detect dynamic-changing events and also to estimate where and for how long the dynamics will be affected.

Permanent Changes In contrast to the two previously mentioned types this one affects the dynamic indefinitely, i.e. walling up one of the doors in a corridor will permanently affect the flow pattern in the corridor. In such a case it is necessary to allow CT-map, T-CT-map and CLiFF-map to forget the old motion patterns and update the existing model to reflect the current motion pattern accurately.

\section{Spatial Dependency}

In work presented in the thesis, I have assumed that there is no prior knowledge about the environment or dynamics therein. However, this assumption is not always valid. In some cases, information about the shape of the environment is available. The information about the shape of the environment can be used either for building a prior for a flow model or improving the quality of map reconstruction. 
Prior for Flow Model A spatial structure of the environment affects the flow behaviour (e.g. a flow of people will typically go along the wall, not through it). Information about the correlation between the elements of the environment and typical flow patterns can be used to construct a set of prior distributions describing the flow in the environment. A possible starting point for incorporating a prior is the usage of social force model [30]. The social force model provides information about human behaviour concerning other objects in the environment. Such information can help to build a prior map, which later on would be updated during the robot's operation. The key benefit is to speed up the mapping process. However, there is also a drawback, in cases when the prior is wrong it might require a substantial amount of data to minimise its impact on the resulting map.

Influence of the Environment on Map Reconstruction An initial study of the impact of spatial information on the process of building a spatially dense CLiFF-map is discussed in Sec. 4.7.3. This initial study emphasises how the presence of the obstacles affect the distances between the observed and unobserved locations. However, the fact that obstacles spatially separate parts of the environment is not the only reason to include the information about the shape of the environment in map reconstruction process. The shape of the environment also influences what flow configurations are plausible, e.g. a flow of people across the corridor is substantially less likely then flow of people along the corridor.

In core, these two problems can be described as enhancing the model of dynamics with spatial information. However, the mechanism and purpose are substantially different. Using spatial information as a prior for a flow model should in the first place help to speed up the mapping process. In case of map reconstruction, the spatial information helps to remove faulty estimates.

\subsubsection{Prospective Applications}

Further research directions that may stem from the contribution presented in the thesis are application oriented. In this section I highlight two areas of application which can benefit the most from the CT-map, T-CT-map, CLiFF-map: planning and motion prediction.

\section{Application for Planning}

Motion planning is the area where the information about the flow can have a significant impact.

In chapter 5 we show an example of motion planner building a flow compliant trajectories. Such trajectories are important in the field of Human-Robot Interaction (HRI), where the aim is to equip the robot with socially acceptable 
behaviours. This initial study should be followed by an in-depth evaluation of how people react to robots able to follow the flow.

HRI is not the only area where it is necessary to evaluate the benefit of flow maps. Another research area where flow information is beneficial is motion planning for aerial and surface vehicles. In this area, the major focus is on evaluation how CLiFF-map can help to minimise the energy consumption and extend the range of robot's operation.

Another area of research covers incorporation of information about flow in planning for gas source localisation. In this case, the goal is to find methods which can speed up gas source localisation by combining information about the global flow of air with gas concentration measurements.

\section{Application for Motion Prediction}

Another research direction is to investigate the applicability of the introduced representations for improvement of motion prediction. The motion prediction algorithms attempt to estimate the future pose of moving objects in the vicinity of the robot. An intensively investigated aspect in the area of motion prediction is long-term predictions.

Gindele et al. [28] address this problem combining Bayesian Occupancy Filter with information about the shape of the environment. In this work, the authors attempt to estimate the probability of cells being occupied based on the observations of past changes of occupancy. Furthermore, in order to focus the prediction only in the areas where the motion is possible, the authors include the information about the obstacles in the environment. Using the information about the shape of the environment helps in extending the range of the predictions and also focus the prediction in these parts of the environment where the motion is possible (e.g. corridors). The combination of CT-map and T-CT-map with work of Gindele et al. [28] might lead for improved prediction in cases where there are not enough obstacles in the environment to affect the prediction process (i.e. open spaces) or in situations where the shape of the environment can still lead to ambiguous predictions (i.e. Y-shaped intersections). In these cases, CT-map and T-CT-map provide a structure of flow which can bias the prediction in this same way as the information about the obstacles in the environment.

Another example of a method addressing the problem of long-term motion predictions is presented by Ziebart et al. [80]. In this work, the authors collect the information about the trajectories followed by people in the environment and use it later to build a cost function which allows predicting the trajectories in the future. However, in large complex environments, it will be very difficult to obtain a set of complete trajectories. Therefore, considering the robustness of CLiFF-map against incomplete data, an important research direction is to investigate how to use CLiFF-map for long-term motion predictions. 



\section{Bibliography}

[1] S. Ali and M. Shah. A lagrangian particle dynamics approach for crowd flow segmentation and stability analysis. In 2007 IEEE Conference on Computer Vision and Pattern Recognition, pages 1-6, June 2007. (Cited on page 22.)

[2] Mihael Ankerst, Markus M. Breunig, Hans peter Kriegel, and Jörg Sander. OPTICS: Ordering Points To Identify the Clustering Structure. In Proceedings of the 1999 ACM SIGMOD International Conference on Management of Data, number 12 in SIGMOD '99, pages 49-60, New York, NY, USA, 1999. ACM Press. (Cited on page 68.)

[3] Daniel Arbuckle, Andrew Howard, and Maja Matarić. Temporal occupancy grids: a method for classifying the spatio-temporal properties of the environment. In IEEE/RSJ International Conference on Intelligent Robots and Systems, volume 1, pages 409-414, 2002. (Cited on pages 16 and 17.)

[4] David Arthur and Sergei Vassilvitskii. k-means++: The advantages of careful seeding. In Proceedings of the eighteenth annual ACM-SIAM symposium on Discrete algorithms, pages 1027-1035. Society for Industrial and Applied Mathematics, 2007. (Cited on page 68.)

[5] S. R. Balaji and S. Karthikeyan. A survey on moving object tracking using image processing. In $201711^{\text {th }}$ International Conference on Intelligent Systems and Control (ISCO), pages 469-474, January 2017. (Cited on page 22.)

[6] Philippe Beaucage, Michael C. Brower, and Jeremy Tensen. Evaluation of four numerical wind flow models for wind resource mapping. Wind Energy, 17(2):197-208, 2014. (Cited on page 21.)

[7] Victor Hernandez Bennetts, Tomasz Piotr Kucner, Erik Schaffernicht, Patrick P. Neumann, Han Fan, and Achim J. Lilienthal. Probabilistic air flow modelling using turbulent and laminar characteristics for ground and 
aerial robots. IEEE Robotics and Automation Letters, 2017. (Cited on pages 21 and 22.)

[8] Victor Hernandez Bennetts, Erik Schaffernicht, Achim J. Lilienthal, Han Fan, Tomasz Piotr Kucner, Lena Andersson, and Anders Johansson. Towards occupational health improvement in foundries through dense dust and pollution monitoring using a complementary approach with mobile and stationary sensing nodes. In Intelligent Robots and Systems (IROS), 2016 IEEE/RSJ International Conference on, pages 131-136, Daejeon, Korea, 2016. IEEE, IEEE. (Cited on pages 98 and 99.)

[9] Maren Bennewitz, Wolfram Burgard, Grzegorz Cielniak, and Sebastian Thrun. Learning motion patterns of people for compliant robot motion. The International Journal of Robotics Research, 24(1):31-48, 2005. (Cited on page 22.)

[10] Maren Bennewitz, Wolfram Burgard, and Sebastian Thrun. Adapting navigation strategies using motions patterns of people. In 2003 IEEE International Conference on Robotics and Automation, volume 2, pages 2000-2005 vol.2, Sept 2003. (Cited on page 109.)

[11] Saket Bhardwaj and Ajay Mittal. A survey on various edge detector techniques. Procedia Technology, 4:220 - 226, 2012. 2nd International Conference on Computer, Communication, Control and Information Technology ( C3IT-2012) on February 25 - 26, 2012. (Cited on page 54.)

[12] L. Biagiotti and C. Melchiorri. Trajectory planning for automatic machines and robots. Springer Science \& Business Media, 2008. (Cited on page 113.)

[13] Peter Biber and Tom Duckett. Dynamic Maps for Long-Term Operation of Mobile Service Robots. In Proceedings of the Robotics: Science \& Systems Conference, pages 17-24, 2005. (Cited on pages 16 and 17.)

[14] R. W. Brockett et al. Asymptotic stability and feedback stabilization. Differential geometric control theory, 27(1), 1983. (Cited on page 112.)

[15] Wolfram Burgard, Cyrill Stachniss, and Dirk Hähnel. Mobile Robot Map Learning from Range Data in Dynamic Environments, pages 3-28. Springer Berlin Heidelberg, Berlin, Heidelberg, 2007. (Cited on pages 16 and 18.)

[16] Guangyu Cao, Hazim Awbi, Runming Yao, Yunqing Fan, Kai Sirén, Risto Kosonen, and Jianshun (Jensen) Zhang. A review of the performance of different ventilation and airflow distribution systems in buildings. Building and Environment, 73:171 - 186, 2014. (Cited on page 21.) 
[17] Tony F. Chan, Gene Howard Golub, and Randall J. LeVeque. Updating formulae and a pairwise algorithm for computing sample variances. In COMPSTAT 1982 5th Symposium held at Toulouse 1982, pages 30-41. Springer, 1982. (Cited on page 40.)

[18] Cheng Chen, Christopher Tay, C Laugier, and Kamel Mekhnacha. Dynamic environment modeling with gridmap: a multiple-object tracking application. In Control, Automation, Robotics and Vision, 2006. ICARCV'06. 9th International Conference on, pages 1-6. IEEE, 2006. (Cited on pages 18 and 19.)

[19] Yizong Cheng. Mean shift, mode seeking, and clustering. IEEE Trans. Pattern Anal. Mach. Intell., 17(8):790-799, August 1995. (Cited on page 67.)

[20] David L Davies and Donald W Bouldin. A cluster separation measure. IEEE transactions on pattern analysis and machine intelligence, 1(2):224227, feb 1979. (Cited on page 68.)

[21] Randall Davis, Howard Shrobe, and Peter Szolovits. What is a knowledge representation? AI magazine, 14(1):17, 1993. (Cited on page 5.)

[22] Arthur P. Dempster, Nan McKenzie Laird, and Donald B. Rubin. Maximum likelihood from incomplete data via the EM algorithm. Journal of the Royal Statistical Society Series B Methodological, 39(1):1-38, 1977. (Cited on page 69.)

[23] Bruce Donald, Patrick Xavier, John Canny, and John Reif. Kinodynamic motion planning. Journal of the ACM (JACM), 40(5):1048-1066, 1993. (Cited on page 107.)

[24] Martin Ester, Hans-Peter Kriegel, Jörg Sander, and Xiaowei Xu. A densitybased algorithm for discovering clusters in large spatial databases with noise. In Proc. KDD, pages 226-231. AAAI Press, 1996. (Cited on page 69.)

[25] L. Fan, Z. Wang, B. Cail, C. Tao, Z. Zhang, Y. Wang, S. Li, F. Huang, $\mathrm{S}$. Fu, and F. Zhang. A survey on multiple object tracking algorithm. In 2016 IEEE International Conference on Information and Automation (ICIA), pages 1855-1862, August 2016. (Cited on page 22.)

[26] Chiara Fulgenzi, Anne Spalanzani, Christian Laugier, and Christopher Tay. Risk based motion planning and navigation in uncertain dynamic environment. INRIA Research Report, 2010. (Cited on page 109.)

[27] Jonathan D. Gammell, Siddhartha Siddhartha S. Srinivasa, and Timothy D. Barfoot. Informed RRT*: Optimal sampling-based path planning 
focused via direct sampling of an admissible ellipsoidal heuristic. In 2014 IEEE/RSJ International Conference on Intelligent Robots and Systems, 2014. (Cited on page 116.)

[28] Tobias Gindele, Sebastian Brechtel, Joachim Schroder, and Rüdiger Dillmann. Bayesian occupancy grid filter for dynamic environments using prior map knowledge. In Intelligent Vehicles Symposium, 2009 IEEE, pages 669-676. IEEE, 2009. (Cited on pages 18, 19, 54, and 125.)

[29] Dirk Hähnel, Rudolph Triebel, Wolfram Burgard, and Sebastian Thrun. Map building with mobile robots in dynamic environments. In IEEE International Conference on Robotics and Automation. IEEE ICRA 2003, volume 2, pages 1557-1563, 2003. (Cited on pages 11 and 16.)

[30] Dirk Helbing and Peter Molnar. Social force model for pedestrian dynamics. Physical review E, 51(5):4282, 1995. (Cited on page 124.)

[31] S. Karaman and E. Frazzoli. Sampling-based algorithms for optimal motion planning. 30, 2011. (Cited on pages 109 and 111.)

[32] S. Karaman and E. Frazzoli. Sampling-based optimal motion planning for non-holonomic dynamical systems. 2013. (Cited on pages 111, 113, and 114.)

[33] Shinsuke Kato and Shuzo Murakami. New ventilation efficiency scales based on spatial distribution of contaminant concentration aided by numerical simulation. American Society of Heating, Refrigerating and Airconditioning Engineers (ASHRAE) Transactions, pages 09-330, 1988. (Cited on page 21.)

[34] Inyoung Ko, Beobkyoom Kim, and Frank C. Park. Randomized path planning on vector fields. The International Journal of Robotics Research, 33(13), 2014. (Cited on pages 108, 109, and 112.)

[35] Gideon Kowadlo and R. Andrew Russell. Improving the robustness of naïve physics airflow mapping, using bayesian reasoning on a multiple hypothesis tree. Robotics and Autonomous Systems, 57(6-7):723 - 737, 2009. (Cited on page 21.)

[36] Tomás Krajnik, Jaime Pulido Fentanes, Joao Santos, Keerthy Kusumam, and Tom Duckett. FreMEn: Frequency map enhancement for long-term mobile robot autonomy in changing environments. IEEE Transactions on Robotics, 33(4):964-977, 2015. (Cited on pages 16, 20, and 123.)

[37] Thibault Kruse, Amit Kumar Pandey, Rachid Alami, and Alexandra Kirsch. Human-aware robot navigation: A survey. Robotics and Autonomous Systems, 61(12):1726-1743, 2013. (Cited on page 1.) 
[38] Tomasz Piotr Kucner, Jari Saarinen, Martin Magnusson, and Achim J. Lilienthal. Conditional transition maps: Learning motion patterns in dynamic environments. In 2013 IEEE/RSJ International Conference on Intelligent Robots and Systems, pages 1196-1201, 2013. (Cited on page 20.)

[39] D. Kularatne, S. Bhattacharya, and M. A. Hsieh. Time and energy optimal path planning in general flows. 2016. (Cited on pages 108 and 109.)

[40] Solomon Kullback and Richard A. Leibler. On information and sufficiency. Ann. Math. Statist., 22(1):79-86, 03 1951. (Cited on page 80.)

[41] Stéphanie Lefèvre, Dizan Vasquez, and Christian Laugier. A survey on motion prediction and risk assessment for intelligent vehicles. Robomech Journal, 1(1):1, 2014. (Cited on page 22.)

[42] T. Lolla, Patrick J. H. Jr, and Pierre FJ L. Time-optimal path planning in dynamic flows using level set equations: realistic applications. Ocean Dynamics, 2014. (Cited on pages 108 and 109.)

[43] M. Luber, G. Diego Tipaldi, and K.O. Arras. Place-dependent people tracking. The International Journal of Robotics Research, 30(3):280, 2011. (Cited on pages 16 and 19.)

[44] J Macqueen. Some methods for classification and analysis of multivariate observations. Proceedings of the Fifth Berkeley Symposium on Mathematical Statistics and Probability, 1(233):281-297, 1967. (Cited on page 68.)

[45] Barbara Majecka. Statistical models of pedestrian behaviour in the forum. Master's thesis, University of Edinburgh, Edinburgh, 2009. (Cited on pages 50,77 , and 82 .)

[46] D. Makris and T. Ellis. Learning semantic scene models from observing activity in visual surveillance. IEEE Transactions on Systems, Man, and Cybernetics, Part B (Cybernetics), 35(3):397-408, June 2005. (Cited on page 22.)

[47] Kanti V. Mardia and Peter E. Jupp. Directional Statistics. John Wiley and Sons Ltd., 2008. (Cited on pages 60, 65, and 93.)

[48] Daniel Meyer-Delius, Jürgen Hess, Giorgio Grisetti, and Wolfram Burgard. Temporary maps for robust localization in semi-static environments. In Intelligent Robots and Systems (IROS), 2010 IEEE/RSJ International Conference on, pages 5750-5755. IEEE, 2011. (Cited on page 13.)

[49] Daniel Meyer-Delius Di Vasto. Probabilistic Modeling of Dynamic Environments for Mobile Robots. PhD thesis, Albert-Ludwigs-Universitt Freiburg im Breisgau, Breisgau, 2011. (Cited on pages 16 and 19.) 
[50] Nikos C. Mitsou and Costas S. Tzafestas. Temporal Occupancy Grid for mobile robot dynamic environment mapping. In 2007 Mediterranean Conference on Control and Automation, MED, 2007. (Cited on pages 16 and 18.)

[51] Hans Moravec and Alberto Elfes. High resolution maps from wide angle sonar. In IEEE International Conference on Robotics and Automation, volume 2, pages 116-121, 1985. (Cited on page 15.)

[52] B. T. Morris and M. M. Trivedi. A survey of vision-based trajectory learning and analysis for surveillance. IEEE Transactions on Circuits and Systems for Video Technology, 18(8):1114-1127, August 2008. (Cited on page 22.)

[53] Patrick Neumann, Sahar Asadi, Achim J. Lilienthal, Matthias Bartholmai, and Jochen Schiller. Micro-Drone for Wind Vector Estimation and Gas Distribution Mapping. IEEE Robotics and Automation Magazine, 2012. (Cited on page 6.)

[54] Patrick P. Neumann, Victor Hernandez Bennetts, Achim J. Lilienthal, Matthias Bartholmai, and Jochen H. Schiller. Gas source localization with a micro-drone using bio-inspired and particle filter-based algorithms. Advanced Robotics, 27(9):725-738, 2013. (Cited on page 89.)

[55] Simon. T O'Callaghan, Surya PN Singh, Alen Alempijevic, and Fabio T. Ramos. Learning navigational maps by observing human motion patterns. In 2011 IEEE International Conference on Robotics and Automation, 2011. (Cited on page 109.)

[56] M. Otte, W. Silva, and E. Frew. Any-time path-planning: Time-varying wind field+ moving obstacles. 2016. (Cited on pages 108 and 109.)

[57] L. Palmieri and Kai O. Arras. Distance metric learning for rrt-based motion planning with constant-time inference. 2015. (Cited on page 111.)

[58] Luigi Palmieri and Kai O. Arras. Distance metric learning for RRT-based motion planning for wheeled mobile robots. In IROS 2014 Workshop on Machine Learning in Planning and Control of Robot Motion, Chicago, USA, 2014. (Cited on pages 112 and 113.)

[59] Luigi Palmieri, Sven Koenig, and Kai O Arras. RRT-based nonholonomic motion planning using any-angle path biasing. In 2016 IEEE International Conference on Robotics and Automation (ICRA), 2016. (Cited on pages 111 and 114.)

[60] Luigi Palmieri, Tomasz P. Kucner, Martin Magnusson, Achim J. Lilienthal, and Kai O. Arras. Kinodynamic motion planning on Gaussian mixture 
fields. In Proceedings - IEEE International Conference on Robotics and Automation, 2017. (Cited on pages 1, 6, and 107.)

[61] Surajit Ray and Bruce G. Lindsay. The topography of multivariate normal mixtures. Annals of Statistics, 33(5):2042-2065, 2005. (Cited on page 70.)

[62] Matteo Reggente and Achim J. Lilienthal. The 3D-kernel DM+V/W algorithm: Using wind information in three dimensional gas distribution modelling with a mobile robot. In Proceedings of IEEE Sensors, pages 999-1004. IEEE, November 2010. (Cited on page 21.)

[63] Jorge Rios-Martinez, Anne Spalanzani, and Christian Laugier. Understanding human interaction for probabilistic autonomous navigation using Risk-RRT approach. In 2011 IEEE/RSJ International Conference on Intelligent Robots and Systems, 2011. (Cited on page 109.)

[64] Leopoldo Rodriguez, J. A. Cobano, and Anibal Ollero. Wind field estimation and identification having shear wind and discrete gusts features with a small UAS. In IEEE/RSJ International Conference on Intelligent Robots and Systems, Daejeon, Korea, 2016. IEEE. (Cited on page 21.)

[65] Anandarup Roy, Swapan K. Parui, and Utpal Roy. SWGMM: a semiwrapped Gaussian mixture model for clustering of circular-linear data. Pattern Analysis and Applications, oct 2014. (Cited on pages 61, 64, and 69.)

[66] Jari Saarinen, Henrik Andreasson, and Achim J. Lilienthal. Independent Markov chain occupancy grid maps for representation of dynamic environment. In 2012 IEEE/RSJ International Conference on Intelligent Robots and Systems, Vilamoura, 2012. (Cited on pages 16, 19, 46, and 50.)

[67] Jari Saarinen, Henrik Andreasson, Todor Stoyanov, Juha Ala-Luhtala, and Achim J. Lilienthal. Normal distributions transform occupancy maps: Application to large-scale online 3D mapping. In Robotics and Automation (ICRA), 2013 IEEE International Conference on, pages 2233-2238. IEEE, May 2013. (Cited on page 40.)

[68] David W. Scott. On optimal and data-based histograms. Biometrika, 66(3):605-610, 1979. (Cited on pages 86 and 89.)

[69] Bernard W Silverman. Density estimation for statistics and data analysis, volume 26. CRC press, 1986. (Cited on page 67.)

[70] Publisher Taylor and Khosrow Dehnad. Density Estimation for Statistics and Data Analysis, volume 26. CRC press, 2012. (Cited on page 67.) 
[71] Sebastian Thrun. Robotic Mapping: A Survey. In Gerhard Lakemeyer and Bernhard Nebel, editors, Science, volume 298, pages 1-35. Morgan Kaufmann Publishers Inc., February 2002. (Cited on page 16.)

[72] Dizan Vasquez, Billy Okal, and Kai O. Arras. Inverse reinforcement learning algorithms and features for robot navigation in crowds: an experimental comparison. In 2014 IEEE/RSJ International Conference on Intelligent Robots and Systems, pages 1341-1346, Chicago, USA, Sept 2014. (Cited on pages 104 and 114.)

[73] Chieh-Chih Wang and Cuck Thorpe. Simultaneous Localization and Mapping with Detection and Tracking of Moving Objects. In Proceedings of the IEEE International Conference on Robotics and Automation (ICRA), volume 3, pages 2918-2924, 2002. (Cited on page 16.)

[74] Qing Wang, Sanjeev R Kulkarni, and Sergio Verdú. Divergence estimation for multidimensional densities via k-nearest-neighbor distances. IEEE Transactions on Information Theory, 55(5):2392-2405, 2009. (Cited on pages 78,79 , and 95.$)$

[75] Z. Wang, P. Jensfelt, and J. Folkesson. Multi-scale conditional transition map: Modeling spatial-temporal dynamics of human movements with local and long-term correlations. In 2015 IEEE/RSJ International Conference on Intelligent Robots and Systems (IROS), pages 6244-6251, September 2015. (Cited on page 20.)

[76] Zhan Wang, Rares Ambrus, Patric Jensfelt, and John Folkesson. Modeling motion patterns of dynamic objects by IOHMM. In 2014 IEEE/RSJ International Conference on Intelligent Robots and Systems, Chicago, 2014. (Cited on page 20.)

[77] Denis F. Wolf and Gaurav S. Sukhatme. Mobile robot simultaneous localization and mapping in dynamic environments. Autonomous Robots, 19(1):53-65, 2005. (Cited on page 16.)

[78] Patrick G Xavier. Provably-good approximation algorithms for optimal kinodynamic robot motion plans. Technical report, Cornell University, 1992. (Cited on page 107.)

[79] Xiaoping Zheng, Tingkuan Zhong, and Mengting Liu. Modeling crowd evacuation of a building based on seven methodological approaches. Building and Environment, 44(3):437-445, 2009. (Cited on page 12.)

[80] Brian D. Ziebart, Nathan Ratliff, Garratt Gallagher, Christoph Mertz, Kevin Peterson, J. Andrew Bagnell, Martial Hebert, Anind K. Dey, and Siddhartha Srinivasa. Planning-based prediction for pedestrians. In 2009 IEEE/RSJ International Conference on Intelligent Robots and Systems, IROS 2009, pages 3931-3936. IEEE, oct 2009. (Cited on page 125.) 


\title{
*Acknowledgements
}

\author{
(...) to write one your own sentence, \\ you have to read a thousand sentences of other people. \\ - Ryszard Kapuściński
}

English is not my first language and expressing feelings in it would not sound sincere. Therefore, the original acknowledgements are in Polish and here you can find the translation in English.

When I was a teenager, I attended a science fair in Wroclaw. During the fair, I have listened to a lecture about an autonomous robot localising sources of ionising radiation. That lecture has started my interest in robotics. Therefore, it the first place I would like to express my gratitude to this lecturer, whose name I do not remember any more. He has unknowingly shaped my whole life.

My biggest thank you goes to Prof. Achim Lilienthal, dr Martin Magnusson and $\mathrm{dr}$ Jari Saarinen. These three people who guided me through my time as a PhD student. I am grateful to prof Lilienthal for trust in my research. I am thankful to dr Magnusson for unwavering support and numerous discussions about my ideas. I want to say thank you to dr Saarinen for inspiration. Finally, I want to also thank dr Janusz Jakubiak, my supervisor during my bachelor and master thesis.

I want to also express my gratitude to other wonderful researchers from Örebro University. Many thanks to Dr Todor Stoyanov for everlasting help in solving big and small software problems, for long and fruitful scientific discussions and multiple joint trips. I am also thankful to dr Erik Schaffernicht for an introduction to the world of machine learning and dr Victor Hernandez Bennetts for joint work on wind flow modelling.

For a more significant part of my time as a PhD student, I have spent working for SPENCER project. The memories of this project will stay with me for long years. I will especially remember Luigi Palmieri and Timm Linder. Thank you for the night shift at the airport!

No trip is genuinely lonely. In this place, I would like to say thank you to my two fellow PhD students, which whom I have shared the struggle for a long time: dr Iran Mansouri and Štefanowi Konečny. Their understanding and support were invaluable. I want especially thank Iran for countless discussions 
which topics reached beyond a daily life of a PhD student. Thanks to these discussions I started to look deeper and broader on the reality. I also want to thank Uwe, Lia, Marcello, Fabien, Jenifer and Mathias for helping me to keep a balance between work and entertainment. I am also grateful to Ewelina and Gosia for friendship and support.

I want to especially say thank you to my parents: Bożena and Andrzej Kucner. They have thought me what is right and how to live a good life. They have inspired me with a passion for science and constant development. They have always supported me in spite of circumstances. I also want to say thank you to my sister, Dominika Kucner, who had a significant impact on the development of my personality.

In the end, I want to express my gratitude to Matylda Czypicka. She helped me to survive the difficult time of thesis writing, helped me to believe in my self and reach the goal. Her presence makes all the sad moments short and all the good moments long. She has painted my life in bright and warm colours.

I have managed to thank only a small number of people whos impact on my life was the greatest. However, they are not the only one whom I want to thank. I want to say thank you to everyone whom I have met in my life. Thanks to your help I have grown and developed. Thanks to you I have reached my goal. Without you all, without your input, I would not be who I am now. 


\section{Publications in the series Örebro Studies in Technology}

I. Bergsten, Pontus (200I) Observers and Controllers for Takagi - Sugeno Fuzzy Systems. Doctoral Dissertation.

2. Iliev, Boyko (2002) Minimum-time Sliding Mode Control of Robot Manipulators. Licentiate Thesis.

3. Spännar, Jan (2002) Grey box modelling for temperature estimation. Licentiate Thesis.

4. Persson, Martin (2002) A simulation environment for visual servoing. Licentiate Thesis.

5. Boustedt, Katarina (2002) Flip Chip for High Volume and Low Cost-Materials and Production Technology. Licentiate Thesis.

6. Biel, Lena (2002) Modeling of Perceptual Systems - A Sensor Fusion Model with Active Perception. Licentiate Thesis.

7. Otterskog, Magnus (2002) Produktionstest av mobiltelefonantenner i mod-växlande kammare. Licentiate Thesis.

8. Tolt, Gustav (2003) Fuzzy-Similarity-Based Low-level Image Processing. Licentiate Thesis.

9. Loutfi, Amy (2003) Communicating Perceptions: Grounding Symbols to Artificial Olfactory Signals. Licentiate Thesis.

Io. Iliev, Boyko (2004) Minimum-time Sliding Mode Control of Robot Manipulators. Doctoral Dissertation.

I I. Pettersson, Ola (2004) Model-Free Execution Monitoring in Behavior-Based Mobile Robotics. Doctoral Dissertation.

I2. Överstam, Henrik (2004) The Interdependence of Plastic Behaviour and Final Properties of Steel Wire, Analysed by the Finite Element Metod. Doctoral Dissertation.

I3. Jennergren, Lars (2004) Flexible Assembly of Ready-to-eat Meals. Licentiate Thesis.

I4. Jun, Li (2004) Towards Online Learning of Reactive Behaviors in Mobile Robotics. Licentiate Thesis.

I 5. Lindquist, Malin (2004) Electronic Tongue for Water Quality Assessment. Licentiate Thesis.

I6. Wasik, Zbigniew (2005) A Behavior-Based Control System for Mobile Manipulation. Doctoral Dissertation. 
17. Berntsson, Tomas (2005) Replacement of Lead Baths with Environment Friendly Alternative Heat Treatment Processes in Steel Wire Production. Licentiate Thesis.

I8. Tolt, Gustav (2005) Fuzzy Similarity-based Image Processing. Doctoral Dissertation.

I9. Munkevik, Per (2005) "Artificial sensory evaluation appearance-based analysis of ready meals". Licentiate Thesis.

20. Buschka, Pär (2005) An Investigation of Hybrid Maps for Mobile Robots. Doctoral Dissertation.

2I. Loutfi, Amy (2006) Odour Recognition using Electronic Noses in Robotic and Intelligent Systems. Doctoral Dissertation.

22. Gillström, Peter (2006) Alternatives to Pickling; Preparation of Carbon and Low Alloyed Steel Wire Rod. Doctoral Dissertation.

23. Li, Jun (2006) Learning Reactive Behaviors with Constructive Neural Networks in Mobile Robotics. Doctoral Dissertation.

24. Otterskog, Magnus (2006) Propagation Environment Modeling Using Scattered Field Chamber. Doctoral Dissertation.

25. Lindquist, Malin (2007) Electronic Tongue for Water Quality Assessment. Doctoral Dissertation.

26. Cielniak, Grzegorz (2007) People Tracking by Mobile Robots using Thermal and Colour Vision. Doctoral Dissertation.

27. Boustedt, Katarina (2007) Flip Chip for High Frequency Applications - Materials Aspects. Doctoral Dissertation.

28. Soron, Mikael (2007) Robot System for Flexible 3 D Friction Stir Welding. Doctoral Dissertation.

29. Larsson, Sören (2008) An industrial robot as carrier of a laser profile scanner. - Motion control, data capturing and path planning. Doctoral Dissertation.

30. Persson, Martin (2008) Semantic Mapping Using Virtual Sensors and Fusion of Aerial Images with Sensor Data from a Ground Vehicle. Doctoral Dissertation.

3I. Andreasson, Henrik (2008) Local Visual Feature based Localisation and Mapping by Mobile Robots. Doctoral Dissertation.

32. Bouguerra, Abdelbaki (2008) Robust Execution of Robot Task-Plans: A Knowledge-based Approach. Doctoral Dissertation. 
33. Lundh, Robert (2009) Robots that Help Each Other: Self-Configuration of Distributed Robot Systems.

Doctoral Dissertation.

34. Skoglund, Alexander (2009) Programming by Demonstration of Robot Manipulators. Doctoral Dissertation.

35. Ranjbar, Parivash (2009) Sensing the Environment: Development of Monitoring Aids for Persons with Profound Deafness or Deafblindness. Doctoral Dissertation.

36. Magnusson, Martin (2009) The Three-Dimensional NormalDistributions Transform - an Efficient Representation for Registration, Surface Analysis, and Loop Detection. Doctoral Dissertation.

37. Rahayem, Mohamed (2010) Segmentation and fitting for Geometric Reverse Engineering. Processing data captured by a laser profile scanner mounted on an industrial robot.

Doctoral Dissertation.

38. Karlsson, Alexander (2010) Evaluating Credal Set Theory as a Belief Framework in High-Level Information Fusion for Automated Decision-Making. Doctoral Dissertation.

39. LeBlanc, Kevin (2010) Cooperative Anchoring - Sharing Information About Objects in Multi-Robot Systems. Doctoral Dissertation.

40. Johansson, Fredrik (2010) Evaluating the Performance of TEWA Systems. Doctoral Dissertation.

4I. Trincavelli, Marco (2010) Gas Discrimination for Mobile Robots. Doctoral Dissertation.

42. Cirillo, Marcello (2010) Planning in Inhabited Environments: Human-Aware Task Planning and Activity Recognition. Doctoral Dissertation.

43. Nilsson, Maria (2010) Capturing Semi-Automated Decision Making: The Methodology of CASADEMA. Doctoral Dissertation.

44. Dahlbom, Anders (20I I) Petri nets for Situation Recognition. Doctoral Dissertation.

45. Ahmed, Muhammad Rehan (20I I) Compliance Control of Robot Manipulator for Safe Physical Human Robot Interaction. Doctoral Dissertation.

46. Riveiro, Maria (20I I) Visual Analytics for Maritime Anomaly Detection. Doctoral Dissertation. 
47. Rashid, Md. Jayedur (20 I I) Extending a Networked Robot System to Include Humans, Tiny Devices, and Everyday Objects. Doctoral Dissertation.

48. Zain-ul-Abdin (20I I) Programming of Coarse-Grained Reconfigurable Architectures. Doctoral Dissertation.

49. Wang, Yan (20I I) A Domain-Specific Language for Protocol Stack Implementation in Embedded Systems. Doctoral Dissertation.

50. Brax, Christoffer (20I I) Anomaly Detection in the Surveillance Domain. Doctoral Dissertation.

5I. Larsson, Johan (20I I) Unmanned Operation of Load-Haul-Dump Vehicles in Mining Environments. Doctoral Dissertation.

52. Lidström, Kristoffer (20I2) Situation-Aware Vehicles: Supporting the Next Generation of Cooperative Traffic Systems. Doctoral Dissertation.

53. Johansson, Daniel (2012) Convergence in Mixed Reality-Virtuality Environments. Facilitating Natural User Behavior. Doctoral Dissertation.

54. Stoyanov, Todor Dimitrov (2012) Reliable Autonomous Navigation in Semi-Structured Environments using the Three-Dimensional Normal Distributions Transform (3D-NDT). Doctoral Dissertation.

55. Daoutis, Marios (2013) Knowledge Based Perceptual Anchoring: Grounding percepts to concepts in cognitive robots. Doctoral Dissertation.

56. Kristoffersson, Annica (2013) Measuring the Quality of Interaction in Mobile Robotic Telepresence Systems using Presence, Spatial Formations and Sociometry. Doctoral Dissertation.

57. Memedi, Mevludin (20I4) Mobile systems for monitoring Parkinson's disease. Doctoral Dissertation.

58. König, Rikard (2014) Enhancing Genetic Programming for Predictive Modeling. Doctoral Dissertation.

59. Erlandsson, Tina (2014) A Combat Survivability Model for Evaluating Air Mission Routes in Future Decision Support Systems. Doctoral Dissertation.

60. Helldin, Tove (20I4) Transparency for Future Semi-Automated Systems. Effects of transparency on operator performance, workload and trust. Doctoral Dissertation. 
6I. Krug, Robert (20I4) Optimization-based Robot Grasp Synthesis and Motion Control. Doctoral Dissertation.

62. Reggente, Matteo (2014) Statistical Gas Distribution Modelling for Mobile Robot Applications. Doctoral Dissertation.

63. Längkvist, Martin (20I4) Modeling Time-Series with Deep Networks. Doctoral Dissertation.

64. Hernández Bennetts, Víctor Manuel (2015) Mobile Robots with In-Situ and Remote Sensors for Real World Gas Distribution Modelling. Doctoral Dissertation.

65. Alirezaie, Marjan (2015) Bridging the Semantic Gap between Sensor Data and Ontological Knowledge. Doctoral Dissertation.

66. Pashami, Sepideh (2OI 5) Change Detection in Metal Oxide Gas Sensor Signals for Open Sampling Systems. Doctoral Dissertation.

67. Lagriffoul, Fabien (2016) Combining Task and Motion Planning. Doctoral Dissertation.

68. Mosberger, Rafael (2016) Vision-based Human Detection from Mobile Machinery in Industrial Environments. Doctoral Dissertation.

69. Mansouri, Masoumeh (2016) A Constraint-Based Approach for Hybrid Reasoning in Robotics. Doctoral Dissertation.

70. Albitar, Houssam (2016) Enabling a Robot for Underwater Surface Cleaning. Doctoral Dissertation.

71. Mojtahedzadeh, Rasoul (2016) Safe Robotic Manipulation to Extract Objects from Piles: From 3 D Perception to Object Selection. Doctoral Dissertation.

72. Köckemann, Uwe (20I6) Constraint-based Methods for Human-aware Planning. Doctoral Dissertation.

73. Jansson, Anton (2016) Only a Shadow. Industrial Computed Tomography Investigation, and Method Development, Concerning Complex Material Systems. Licentiate Thesis.

74. Sebastian Hällgren (20I7) Some aspects on designing for metal Powder Bed Fusion. Licentiate Thesis.

75. Junges, Robert (2017) A Learning-driven Approach for Behavior Modeling in Agent-based Simulation. Doctoral Dissertation.

76. Ricão Canelhas, Daniel (20I7) Truncated Signed Distance Fields Applied To Robotics. Doctoral Dissertation. 
77. Asadi, Sahar (20I7) Towards Dense Air Quality Monitoring: Time-Dependent Statistical Gas Distribution Modelling and Sensor Planning. Doctoral Dissertation.

78. Banaee, Hadi (2018) From Numerical Sensor Data to Semantic Representations: A Data-driven Approach for Generating Linguistic Descriptions. Doctoral Dissertation.

79. Khaliq, Ali Abdul (2018) From Ants to Service Robots: an Exploration in Stigmergy-Based Navigation Algorithms. Doctoral Dissertation.

80. Kucner, Tomasz Piotr (2018) Probabilistic Mapping of Spatial Motion Patterns for Mobile Robots. Doctoral Dissertation. 